\title{
Scholarly Communication and Academic Presses
}

\author{
Proceedings of the \\ International Conference \\ 22 March 2001, University of Florence, Italy
}

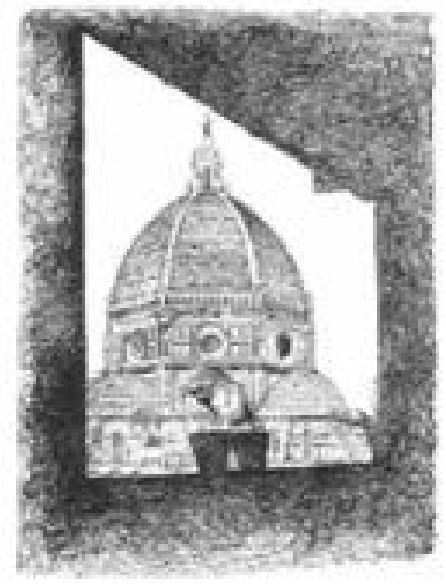

Edited by Anna Maria Tammaro 


\title{
Scholarly Communication and Academic Presses
}

\author{
Proceedings of the \\ International Conference \\ 22 March 2001, University of Florence, Italy
}

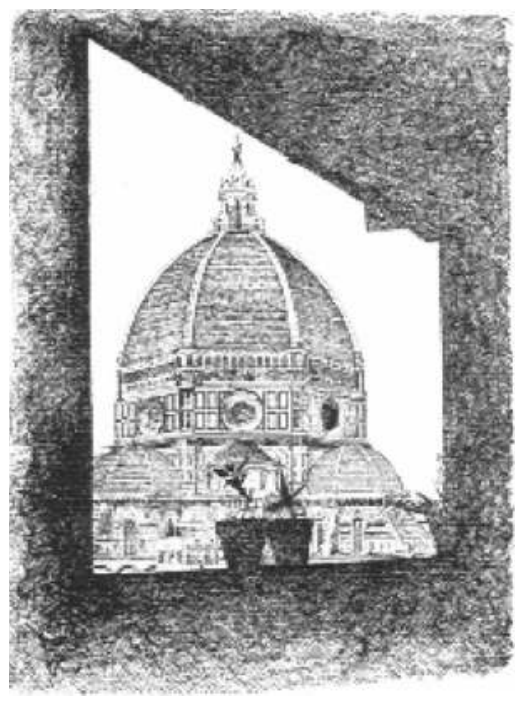

Edited by Anna Maria Tammaro 
Scholarly Communication and Academic Presses: proceedings of the international conference, 22 March, 2001, University of Florence, Italy / edited by Anna Maria Tammaro ; organised by Università degli Studi di Firenze. - Firenze : Firenze University Press, 2002.

http://epress.unifi.it

ISBN 88-8453-049-0

070.594 (ed. 20)

Scholarly publishing - Congresses

Digital publishing - Universities

Print on demand is available

(C) 2002 Firenze University Press

Firenze University Press

Borgo Albizi, 28

50122 Firenze, Italy

http://epress.unifi.it/

Printed in Italy 


\section{Table of contents}

Scholarly Communication and Academic Presses: an Introduction

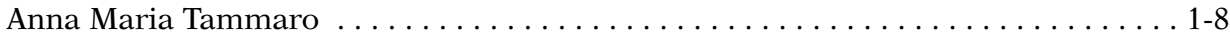

Tinkers and Tailors: complementarity between university presses and libraries

Michael A. Keller . . . . . . . . . . . . . . . . . . . . . . . . . . . . . . . 9-16

The Self-Archiving Initiative

Stevan Harnad . . . . . . . . . . . . . . . . . . . . . . . . . . . . . . 17-23

The Roquade Project. The library as a catalyst of change in scholarly publishing

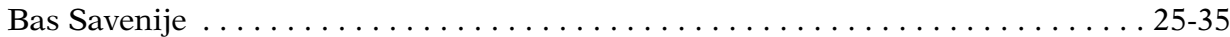

Legal Deposit of Electronic Publications

Giovanni Bergamin . . . . . . . . . . . . . . . . . . . . . . .

EMIS - the Involvement of EMS in Publishing Mathematics

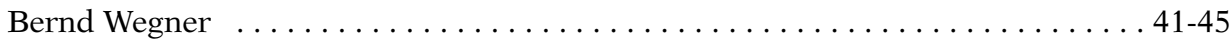

The Role of the Publisher in Scholarly Communication

Anthony Watkinson . . . . . . . . . . . . . . . . . . . . . . . . . . 47-55

Change in Universities: the effects for libraries, academics and pub-

lishers

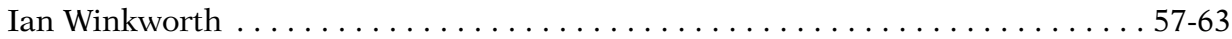

Publication vs Communication?: Searching for an identity for the

University of Florence e-Press

Anna Maria Tammaro

"Reti Medievali": an initiative of new communication of historical knowledge

Andrea Zorzi . . . . . . . . . . . . . . . . . . . . . . . $75-78$

A Framework for Italian Digital Libraries: the Dafne Project

Jacopo Di Cocco, Laura Tallandini, Anna Maria Tammaro . . . . . . . . . . . . 79-85

The Limits of Innovation: A publisher's view about the impact of digital publishing on academic. communication and research diffusion 
JOP. Journal of the Pancreas: a model for the dissemination of scientific information and sharing of knowledge in the field of Pancreatoloy and Diabetology

Raffaele Pezzilli, Generoso Uomo, Antonio Maria Morselli-Labate

E-communication among Italian Zoologists: two years of experience with the "Vertebrati" mailing list

Claudio Gnoli

Scholarly Communication and Academic Presses: "The Nexus Network Journal"

Kim Williams

Academic Presses and Integrated Systems : towards a new perspective

Antonella Cosetti, Gian Franco Greppi, Massimo Valdini

\section{Conclusions}

Hans Geleijnse 


\title{
Scholarly Communication and Academic Presses: an Introduction
}

\author{
Anna Maria Tammaro \\ Coordinator of Firenze University Press \\ University of Florence \\ Professor of Library and Information Science \\ University of Parma, Italy \\ E-mail address: tammaro@unifi.it, annamaria.tammaro@unipr.it
}

Firenze University Press is the digital press of the University of Florence and has hosted an international discussion among scholars, scholarly societies, university administrators, librarians and publishers with the aim of identifying the challenges in scholarly communication and the opportunities for change, many of which are suggested by digital and network technologies. The objective of the meeting was to identify concrete ways for implementing proposals to foster the international scientific co-operation of universities and academic societies. Against the background of an assumed tendency towards knowledge monopolies, caused by the protection of intellectual property rights by a few publishers, the experts in the Conference discussed the potential of the academic e-press with respect to improving scientific article collection and treatment as well as facilitating the access to scientific knowledge.

The papers in this volume constitute most of those which were presented at the Florence Conference, held on March $22^{\text {nd }} 2001$. None of the conclusions put forth in these papers is definitive: it is too early for that. But they demonstrate that a substantial amount of learning about how to operate more effectively has already taken place. The papers in this volume then can best be regarded as contributions to the areas of inquiry in a field that is continuing to change very rapidly. The papers will serve if they can provoke thoughts in the reader as well as inform him.

\section{Authors are kings}

The assumption of the speakers was that the business arrangements of the scientific journals for which faculty write, edit, and review must become a major focus of authors, contributors, editors and readers, if scholarly publication is to become affordable again. The most important challenge of the re-appropriation of scholarly communication by authors and academic societies is how to improve scholarship impact, learning and teaching while simultaneously reducing the costs. Containing costs might be accomplished over time, through the effective use of technology to streamline publishing functions while increasing access and value. 
What each example in the Conference has illustrated is the necessity to focus on the needs, aspirations, practices, and qualities of specific knowledge workers as consumers and producers of content. Many scholars have become active users of electronic resources in their scholarly research. However, they are still much less likely to try to publish their own scholarship electronically than they are to access materials electronically for scholarly purposes. Such reluctance, the Conference noted, can be directly attributed to issues surrounding credibility and cultural behaviour.

The question of status was also brought up: are electronic journals as well-regarded as traditional print journals? Conference participants seemed to feel that there was no stigma placed on electronic journals as such, but rather that the acceptance, or lack of acceptance of e-journals, could be attributed to the fact that most e-journals are new journals and it takes time for any new journal to become accepted.

Here the Conference has drawn particular attention to the notion of perceived value and how it relates to academic publication. Central to the perception of value, is the process of publication, imprint, and peer review. Well managed and understood in the print medium, the very nature of the electronic medium -a new environment, in which clear and accepted standards are only now beginning to emerge- has meant that these concerns have been treated with a scepticism appropriate to the academic arena. It is important to realise that the problem of establishing credibility is in one sense circular: because established scholars are suspicious of the medium, they are unwilling to publish in it, and because they do not publish in it, the medium will continue to lack credibility. Young scholars, in particular, are unlikely to take the risk of publishing in an electronic format.

A second concern is over the value that should be ascribed to electronic scholarship. Most members of academic staff go through a periodic (often annual) assessment of their academic performance, and for many, this assessment is tied to a process of awarding merit. A concern over the perceived value of an electronic publication will inevitably lead to decreased usage of this method of dissemination. A further concern is a perception that the electronic text is unstable, and consequently unreliable.

The creator may himself have divided interests. On the one hand he wants to have the largest circulation of his work in order to spread his academic reputation. On the other hand he may want to maximise royalty income. Or indeed he may want to have a compromise solution in which both royalty income and circulation have got some weight.

The requirement for focus on the needs of authors and readers has suggested, as a first result of the discussion in the Conference, that niche publishing and specialised selfarchives will be fruitful areas.

\section{Preservation and text fluidity}

The advancement of knowledge is dependent on access to prior scholarship. And this should alert us to the new possibilities for collaboration between presses and libraries. While research libraries have made significant progress in preserving print publications, there is still a large proportion of unique printed material yet to be treated and a number of additional formats, such as videotapes, sound recordings, and films, whose preservation needs have yet to be addressed in some significant way.

Electronic publishing adds yet another set of complex issues to the archiving and preservation of scholarly works. Electronic scholarly texts can be thought of as ordered collections of digital objects, comprised of such entities as texts, images, audios, and simulations. Specific hardware and software may be required to read them. In such an environment, the archiving challenges are many. 
With libraries no longer owning copies and with the fragility of the electronic media, questions of what should be archived by whom and how, are critical issues that need to be addressed. Despite many unanswered questions and unknown costs, archiving and preserving scholarly publications in all media are critical to any credible system of scholarly publication. Scholarly publications must be archived in a secure manner so as to remain permanently available and, in the case of electronic works, a permanent identifier for citations and links should be provided.

One communication referred to concerns about the preservation of scholarship. All those who publish are, at some level, concerned with archival issues, so it was hardly surprising to learn that National Libraries partners in the Nedlib Project are concerned with the long-term preservation and continued availability of scientific works. This concern is especially marked in relation to the electronic medium, which is both new and significantly different from print. The Nedlib Project will include considerations of migration strategies as well as issues related to version control and to text boundaries, such as referenced objects. The Nedlib Project will yield a list of identifiable strategies (technological and cultural) to deal with these intersecting needs.

One of the points brought up at the digital publishing conference was that the advent of a new medium had given rise to a plethora of new journals. Many of these, however, failed to take into account whether the particular field could support another journal, and many journal failures were blamed on the failure of the new digital medium rather than on the failure of the journal itself. The experience of the Firenze University Press (FUP) has suggested that some academics perceive electronic publications as being texts devoted to communication, that undergo continuous change and adjustment, and that this undermines concerns related to stability and archiving. The FUP will explore the consequences on traditional scholarship associated with the perception of what might best be called "textual fluidity".

The FUP has also examined its options for ensuring textual integrity in a fluid environment. The experience of a University Press will seek to reconcile the need for stability in archiving texts with a recognition that one of the features of the electronic text is that it can be "maintained" and kept up to date in a way which the print medium does not allow. In this context, it becomes possible to speak positively of the electronic text as "dynamic" rather than "unstable".

\section{Unifying small journals}

The successes in technological innovation are those which resonate with a real human need, which genuinely do something better or cheaper, or do something which could not be done before, and which find people and organisations able to apply them. Electronic capabilities should be used, among other things, to:

- provide wide access to scholarship,

- encourage interdisciplinary research,

- and enhance interoperability and searching ability.

Development of common standards will be particularly important in the electronic environment for unifying small journals online, without sacrificing their intellectual independence. This is what the Electronic Library in Mathematics (ELibM) is doing for mathematical science. The existence of the umbrella organisation of the European Mathematical Information Service (EMIS) has made the project viable. EMIS tries to bundle into a joint offer: several literature databases (MATH and MATHDI); freely accessible scientific journals and the pre-print index MPRESS; the project to install the 
EULER search engine for distributed sources; and, most important, the Electronic Library to extend them by innovative components and to distribute them in a system of replicators for the benefit of mathematicians worldwide. It is based on the voluntary supply of input from sources distributed worldwide. The first stream is helping independent mathematics publishers to make the transition to the electronic scene. The second stream is acting as a catalyst for the mathematics publishing community to arrive at a commitment to use a common metadata standard.

\section{Disintermediation}

The ideas of disintermediation are still actively propounded in some circles. Such a disintermediation system is being promoted by the Open Archives Initiative (OAI), an effort that strives for compatibility among e-print services. Harnard, the most discussed speaker at this Conference, has explained how the Open Archives movement renders one sort of primary publishing irrelevant. Researchers publish their findings in order to make an impact on research, not to sell their works. Access-tolls are barriers to research impact. Authors can now free their refereed research papers from all access-tolls by immediately self-archiving them online in their own institutions e-print archives. Open Archives are systems that would build peer-review and abstracting and indexing functions upon discipline- or institution-based e-print services. This should enhance research productivity and impact as well as provide powerful new ways of monitoring and measuring it.

Some of the speakers associated with different discipline areas and similar librarysponsored bodies have asserted that one of their aims is to directly bring author and reader together. Zorzi, Professor of medieval history at University of Florence is director of Reti Medievali, a set of web pages that analyses and promotes the use of computer techniques in the study of medieval history. He well expressed the point of view of scholars who intend to employ new technologies of communication in order to bypass the organisational and economical barriers that condition the traditional press channels for the communication of historical knowledge and in order to favour a wider distribution of personal publications.

Pezzilli, the chief editor in Italy of the large medical journal published online only, the Journal of the Pancreas (JOP), holds the same views. Peer review, identified as the most important factor for the quality of journals, is assured by an online editorial board. For Gnoli, a member of the Secretariat Board, the survival of Vertebrati through the critical phases of its infancy, its consolidation and its two-year long experience, which even in the light of the realised survey results can be viewed as positive, mean that it now represents a precious resource, worthy of investing renewed efforts, and of producing valuable information for the scientific community. The editor of Nexus Network Journal (NNJ), Williams, spoke of her experience which started as a conference series begun as a way to bring together people working on interdisciplinary studies in architecture and mathematics. This journal for architecture and mathematics appeared on the Internet with its first issue in January 1999.

The quest for innovative alternatives disclosed by the digital press, raised new problems for those scholars who decide to get personally engaged in the preparation and editing of these new means of publication.

\section{Actors roles}

The important fact is that there are numerous agents in the chain from the creator of intellectual property to the ultimate users. As Winkworth said, "All this points to rethink- 
ing of old roles and processes in respect of scholarly communication. In the light of these considerations, let us remind ourselves of the traditional scholarly communication process"

\section{Publishers}

It is common in library circles to make a distinction between "commercial publishers", who are bad, and non-profit publishers, who are good. Watkinson tried to define what is this editorial role, "To my mind it has two main components. The first is selection and the second is content development".

In many ways both the function of selection and certification are even more important in the electronic then in the print environment because it is much more easy to put rubbish on the Web. One example is that the publishers have recognised the globalisation of the scholarly discourse. They will need to better consider the protection of the moral rights of integrity and paternity much more actively in the future because the dangers to preserving authenticity will be so much greater than they were in the past.

In spite of the original enthusiasm, digital publishing growth for commercial publishers is facing difficulties and uncertainties. Especially in the academic area, the opinion about the low scientific value of the existing electronic publications has greatly limited the development of digital publishing. Moreover, the technical problems, which still persist in digital library creation from paper publication archives, discourage investments in the electronic publication consulting frameworks. This fact causes a loss of interest in the academic area, too, for electronic publications, which basically are inaccessible from the university librarian systems. In particular in Italy, very few publishers have started publishing on electronic support. In Francesconi's paper the status of digital publishing is discussed from an academic publisher's point of view, as related to the technical obstacles towards electronic documents availability, which is strictly linked to the reliability of document digitalising systems.

\section{Libraries}

Libraries were more active than expected. For librarians, it is ridiculous that the professor gives away copyrights and that the students must pay. How could they defend the users, in particular the students, when every single university will be online on the Web? Librarians really support OAI and some of them have started a University press supporting teachers in publications. Such experiences have been demonstrated within the Conference by Stanford University's HighWire Press and The Project Roquade; other efforts, such as FUP, are being facilitated by local universities and national goverments.

On this point, Keller said that :

"The assumption that publishers and librarians were outmoded floating around at the beginning of the 1990s if it has not disappeared, it is certainly discredited. However, informal publishers and distributors are now on the scene and are unlikely to disappear. It is a more complicated world. And the role of the university libraries evolved from providing more or less clerical support in response to the requests of professors to much more activist roles, sometimes leading or predicting trends in scholarship, also always devoted to the interests of the academic host".

The fundamental role of librarians as institutional managers of access to information sources remains unchanged.

The Roquade Project, initiated by the libraries of the Dutch universities of Utrecht and Delft, is such an initiative. It aims at creating an infrastructure that conglomerates the 
swiftness of publication which hitherto could only be realised by grey publishing, with quality judgement without the serious delay of the traditional review procedures. Roquade offers a wide number of facilities to a broad audience, based on common organisational and technical infrastructure. The project will have benefits for the academic community, as well as for the libraries that take the initiative. Its intended benefits for the academics (both authors and readers) can be summarised as follows:

- Rapidity

- Quality

- Transparency of the reviewing and publishing process

- Positive attitude of scientists towards digital publishing

- Technological innovation

- Fair price

- Usage statistics.

The intended benefits for the library will be:

- Opportunities for applying gained experience of digital publishing and for exploiting the existing infrastructure in order to support the information and communication requirements of researchers;

- Better position in negotiations with publishers active in all fields in order to slow down the rising in subscription rates as well as improving financial benefits for the academic community in the long run;

- International co-operation.

\section{University presses}

University and academic presses can play an important role in supporting academics in publishing. Quality control is essential and crucial. Organisation of peer review is changing. It should be independent because it will help to develop science and technology. University presses today are different from university presses of ten years ago. University presses need support from universities and associations. Most influential authors should use them, thus influencing others. They should take advantage of the experiences of others, the European co-operation launched from Signal Hill wants to take a step in this direction.

Keller asserted that the role of the university press really started as a printer, but has evolved in the last 100 years to that of a real publisher which is always devoted to the interests of the academic host. The political support to university presses is needed for facilitating scholarly communication.

The DAFNE Project, financed by the Italian Government, was begun to develop a model that could serve the scholarly, educational and academic needs of society, facilitating the international discovery and use of Italian digital resources. The aim of this model is that the various stakeholders can collaborate in building a framework from which the transition to a fully electronic environment will be facilitated. This model is based on the interoperability of different information systems and on new alliances between commercial publishers, university presses and libraries. The importance of the DAFNE Project lies in the realisation and fine-tuning of a complete structure which enables the user to have access to the information and document retrieval, prefiguring the National Digital Library and enables the producers of goods and services -even if these are frequently entities of limited size- to take advantage of the "Italian district of electronic publishing".

The objectives of the Project DAFNE are:

- to realise an agent for licensing

- to plan the digitalisation of collections at national level 
- to build an index to Italian scientific periodicals

- to co-ordinate the development of the university presses and the collaboration with publishers.

\section{A look into the future}

It is impossible to predict what the future for academic publishing will look like. Furthermore, it is rather short-sighted to declare a standard for what the future should look like. Therefore, one should not impose one's preferred model upon scientific communication. The academic community should take initiatives that, in a flexible way, facilitate new structures in scientific information processes, which are favourable for the academic community itself and innovate the traditional scholarly communication flow.

With the growing volume of scholarly research, it is increasingly difficult to uncover all of the relevant material published on a given subject. As more scholarship becomes available in digital form, this problem can be surmounted through powerful search systems provided that commercial, technical and legal constraints do not prohibit such searches. Searching, navigating, and linking across titles and across disciplines is essential, since many disciplines have multiple titles that serve them and many problems have multidisciplinary aspects that may lead a researcher to publications in fields as diverse as microbiology, law, economics, and internal medicine. The development of standards is critical to the implementation of cross-field searching and navigating. In addition, given the importance of older literature to the advancement of new knowledge, retrospective works should be digitised and made accessible online.

Cost containment should also continue, through library consortial purchasing of electronic resources, through the academic e-presses, a strategy that appears to be effective in lowering the unit costs of electronic information, and especially through the institutions support of e-print servers for freeing scientific works. Because in the scholarly arena authors and readers are essentially the same community, the new publishers bring insights to their task from their research and service experience. They hope to integrate with the technology some of the processes and thus serve scholarship better and at less cost. Whatever the solution(s), costs must be made to fit within available budgets or the system will fail to provide the information to scholars that they need.

The adoption of appropriate peer review and archival mechanisms by those who publish academic material in electronic form would make electronic publication more acceptable. At heart is the issue of credibility. Any attempt to improve the perceived value of electronic publishing will have to take into account the negative perceptions of electronic scholarly publication. The Conference wishes to recommend a series of strategies to encourage high quality publication in electronic format, and to break the circle of scepticism that surrounds the perception of the electronic medium. Peer review, identified as the most important factor to assuage the reluctance of scholars to publish electronically, is a process that has evolved over many generations of scholars. It has become the cornerstone of academic publication and is something that is highly valued in all scholarly activities (among them the pragmatics of academic review). It is important to recognise that peer review is necessarily, and appropriately, a conservative process and that any new scholarly endeavour will take time to gain general acceptance.

The permanent increase of journal prices leads to more engagement of universities, libraries and societies in low-budget electronic publishing to provide an alternative to high-priced commercial publications. The small publishers have been much slower in making the transition into the online environment than the larger players. A lot of these 
journals have not made the transition at all. Others have an online presence that was created on a shoestring with no institutional commitment for addressing important longevity issues such as metadata, archiving, authentication, electronic commerce and system development. So even though these small titles are an important part of the intellectual landscape in the print world and are among the most affordable ways to access scholarly research, they need help to make a successful transition into the online environment. Since small journals tend to value their editorial integrity very highly, they are hesitant to accept help from any source unless they are considered neutral.

It was suggested by Winkworth that this technology basis will lead to the following key features of publishing in 2005:

- More scholarly publications, with open e-print archives challenging commerce

- Hybrid world of print and electronic publication and delivery

- Research "virtual communities" with access only to accepted members

- Rights management via licenses, not legislation; "fair dealing" confirmed

- Institutional debate over intellectual property rights. 


\title{
Tinkers and Tailors: Complementarity between University Presses and Librairies
}

\author{
Michael A. Keller \\ University Librarian \\ Director of Academic Information Resources \\ Publisher of HighWire Press and Stanford University Press \\ Stanford University, Berkeley, USA \\ E-mail address: makeller@sulmail.stanford.edu
}

\begin{abstract}
This paper will dwell on a few key issues regarding university presses and university libraries, some historical, some drawn from the present and recent past, some perhaps pointing to the future. Common to each issue is the relationship in principle and in actuality of the presses and the libraries in supporting and advancing the missions of the university. What has failed, what has been successful, and what might we do together to make a better future. Since the dawning of the Internet age a few hours ago, the prospects for both sorts of organizations have changed. As each adjusts or morphs to find its niches and then to fill them, what are the prospects for collaborative engagement.
\end{abstract}

Let me start by thanking Anna Maria Tammaro for inviting me to speak at this conference on scholarly communication. The university press of the University of Florence has embarked on a significant new path, that of an electronic press first and foremost. This is a remarkable development that deserves our attention now and in the coming years as it progresses, for it most certainly will progress.

My talk today focuses on the roles of university presses and university libraries, each providing crucial functions in the middle of the chain of scholarly communication, a well populated zone with academic authors at the start of the chain and academic readers at the end of the chain. There are other players in the middle of the chain too, but I may mention them only in passing. And of course one cannot address either the publishing functions or the library functions without taking into account the opportunities and threats provided to the middle players as well as to the chain itself by the presence of networks and various digital technologies.

The title of my talk is intended to provoke consternation and curiosity. Why would one link itinerant craftsmen working mainly in metals with stay-at-home garment makers working almost entirely in cloth? And the Italian translation of the title, Stagnini e Sarti, is even more startling, because the tradition of traveling repairmen of pots and pans is not as pronounced in Italian culture as it is in other ones. Why chose this odd pairing? tion.

Perhaps a closer examination of each element of the pair will help answer the ques-

Tinkers were men who traveled widely in earlier times looking at each village and remote household for work repairing metal objects, particularly pots, pans, and kitchen implements. They were opportunists, seizing upon any possibility to earn a living. In fact, 
some of the opportunities seized were illegal ones. The term tinker carries with it more than a whiff of missing chickens, light fingeredness, and shady dealings. However, for the purposes of this talk, the prime elements of interest are the tinkers roaming in search of opportunities to be seized. The iconography of tinkers shows the cheerful vagabond, roaming hill and tale, looking for work.

Tailors were and are patient stay-at-homes. They take commissions and create garments, often at the specifications of others. Tailors are village folk, attached to a particular social setting. They take measurements, then cut and stitch cloth to make a shirt, a dress, a suit, an ensemble. Tailors are at the beck and call of others, though some are recognized as creative types. As a generality, tailors are pictured in shops, wearing spectacles, working with needle and thread on a piece of clothing, often with a client hovering around them.

There is no obvious link between the work of tinkers and the work of tailors. There is no obvious complementarity either. Their social status is probably comparable, with a slight elevation of the tailor over the tinker, but both are in the broad class of necessary craftsmen in any pre-industrial society.

The analogy I would like to make, admittedly an imperfect one and admittedly one from a particular point of view, is this. Publishers have been like tinkers, roaming the countryside taking advantage of discovered opportunities for the application of their craft. Librarians have been like tailors, taking the measure of the needs of their customers, then sitting patiently to assemble collections from various sorts of disparate things, books, serials, manuscripts, archives, and so forth. And the part of the relationship between presses and libraries which has varied in low order intensity over the years is that of the complementary nature of the one trade to the other. In some times, publishers, particularly those in university presses, have been more explicitly involved with libraries than in other times. It is the odd pairing of tinker and tailor, of publisher and librarian, as well as the imperfection of the analogy which gives a scaffold to this talk. I will take advantage of the $20^{\text {th }}$ century history of university presses and university libraries to bring in the network and digital technologies to this tale and thus provide the potential for another analogy, one of much better complementarity than tinkers and tailors. Now I admit that I speak from the perspective of a librarian, albeit one with publishing responsibilities. And I suspect that publishers listening to this might really want the label tinker to be replaced by another one, say duke or prince.

Most university presses in America developed late in the $19^{\text {th }}$ or early in the $20^{\text {th }}$ century. The term "university press" is indicative of the role they played in their institutions back then. In effect, the presses were printers and distributors for the university as publisher. If a department or school authorized a monograph to be published, they turned to their university press to edit, print, and distribute the work. Distribution was sort of an after thought though, because many of the copies were distributed by the authors and their departments to their colleagues. The university presses were started to make copies of works of scholarship once those works had been selected, in effect refereed, by the home departments (often meaning the department chair) to share the results of scholarship, but just as much to enhance the reputation in the embryonic professional societies. Only later in the $20^{\text {th }}$ century did university presses develop publishing programs of their own, always, it must be noted, under the direction of boards of editors populated entirely by faculty members. And still later in the last century does the prejudice against publishing in one's own university press become common. This turn-about occurs because of the presumption that one's own press would be performing in a kind of vanity press role. One's work is better published by another university's press than one's own in order to demonstrate that one's work is accepted by editorial boards not in the control of local 
academic politics. The irony of this situation which is pervasive today is that one's cronies are as likely to be assembled in the disciplinary groups, the scholarly societies and their tribes, as in one's own institution. Indeed, the professionalization of the professoriate is more likely, in my view, to create cronyism within disciplinary groupings, in scholarly societies, than in departmental settings where so many other sorts of politics seem to keep cronyism at bay. We should note as well that in the latter part of the last century, university presses, or at least some of them, developed publishing programs involving trade and professional publications, works intended for educated readers, but not necessarily works of communication strictly among specialist scholars.

Many works published by university presses were used by libraries for exchanges of titles in order to build better collections for the support of teaching and research. Stanford University Press was established explicitly stating among others the purpose of providing the university libraries with publications to trade with other institutions, especially ones abroad, meaning European universities.

In the same period of time, the $20^{\text {th }}$ century, most university libraries started as organizations mainly reacting to the wishes and needs of the faculty for assembling books and journals requested by them. The work of most university librarians early in the century was more or less clerical, the professional aspects addressed in the literature of librarianship dealing mainly with classification systems, descriptive cataloging principles, and the application of these to local operations. One could assert that a late manifestation of the same drive for comprehensive grasp of the world around us exhibited by the $18^{\text {th }}$ century encyclopedists is the classification of knowledge in systems devised by Panizzi, Harris, Dewey, and an unsung group of specialists in the Library of Congress working at the turn of the century. The use of library classification systems to associate books based on subject is the work of tailors, not tinkers. The use by university libraries of university press books for exchange with other research libraries is a point of connection between the tinkers and the tailors, though there is little evidence of influence by libraries on the publishing projects or programs of the presses. Like tinkers and tailors, the point of connection is a small one. Just past the middle of the $20^{\text {th }}$ century, research librarianship evolved into a complex professional milieu, with professional collection development librarians replacing the "request and acquire" model of the earlier years. With the explosion of research in the Second World War and the even larger explosion of research occasioned by the cold war and the race to space, all phases of librarianship take new roles, more or less independent ones, although always in service of research and teaching. In the years from the middle of the century until the early 1980s, university libraries and a great many college and public libraries provided university presses with the bulk of the sales of their books.

Around 1980, perhaps a little before, something happened to upset this cozy producerconsumer relationship between university presses and university libraries. The commodification of scientific, technical, and medical information in the form of journal articles, issues, and titles, especially by some canny for-profit publishers drove spending to the scientific side of the library book budgets, a process which is still unfolding today. University presses had then and still do today focus on books in the humanities and social sciences, with only a few of them publishing scientific, technical or medical journals at all. So, over the past 20 or 25 years, the university libraries have stopped acquiring more or less automatically every book published by university presses; careful and selection of smaller numbers of books has become the norm. The result of this change has been a serious challenge to all university presses. They have sought to employ new formats, paperbacks mainly, to offer cheaper books to more individuals, and some have added trade and professional books to their publishing programs to eke out a living for- 
merly guaranteed by publishing only scholarly books. In the last decades of the $20^{\text {th }}$ century, all but a few university presses are subsidized operations. In the context of tighter and tighter management of university resources, those who are subsidized become very defensive about their very existence on the one hand, and are likely on the other to overreact by publishing too many titles in hopes of finding a "best seller" every once in a while to justify the many, many books sold in very small numbers of copies. In effect, the tinker was getting squeezed out of the scene by pressures and forces unseen, misunderstood, and implacable. And in this period as well, university libraries use of university press books for exchange purposes was reduced to almost nothing.

To sum up this history in a convenient and therefore suspect manner in order to get on to the possibilities of our times, I assert that the role of the university press started really as a printer, but evolved in the last 100 years to that of a real publisher, always devoted to the interests of the academic host. And the role of the university libraries evolved from providing more or less clerical support in response to the requests of professors to much more activist roles, sometimes leading or predicting trends in scholarship, also always devoted to the interests of the academic host.

To a considerable extent, the tinker/tailor dichotomy applies as an analogy to university presses and university libraries as much now as it did in the early years of the $20^{\text {th }}$ century. University presses still seize opportunities as they wander the hills and dales of academe and university libraries still patiently stitching together collections and services for readers in individual academic institutions. In these times, the ones we live in right now, were it not for a significant alteration to the possibilities for scholarly communication as much as for the continued fiscal pressures of tight budgets and soaring costs in one sector of interest, that of scientific and technical information, tinkers and tailors might have had every reason to be quite concerned about their futures.

The significant change, of course, is the addition of the Internet as a publishing and distribution medium made possible by the easy and cheap availability of network browsers such as Mosaic, Netscape and Internet Explorer, as well as by numerous hypertext features.

You will be relieved that I will drop the tinker and tailor analogy now to discuss the new possibilities and the opportunities offered in this Internet age for scholarly communication, some of which have possibilities of collaboration between university presses and university libraries.

First, here are a few facts of the Internet which are indisputable, I think.

One: the Internet makes it possible for every possessor of a computer with access to network connections to be a distributor of information. There are hundreds of millions of computer owners who could be processors and distributors of information, and thus an informal kind of publisher. The practical result of this possibility is a kind of information chaos on the Internet; I will return to this point in a minute.

Two: another fact is that the ease of applying well-engineered tools to intellectual property -to texts, images, sounds, numeric data, representations of the various building blocks of life, for example- has made possible the entry to the realm of scholarly communication a number of new middle players. One has only to look at the list of supporters of tomorrow's conference on the digital university to see a few of these. Some of these new players have some new ideas about how scholars might communicate with one another and with other readers. Probably the dot.com bubble, conclusively burst, made an efflorescence of new entrants to the chain of scholarly communication; from these days forward there will be fewer new entrants. We will hear later in this program about some challenges to the entrenched players in the middle of the chain, but I think there is little doubt that the essential functions of publishers -selecting, validating, organizing, clarify- 
ing, distributing intellectual property- will persist. And the essential functions of libraries- selecting, organizing, interpreting, distributing, and preserving intellectual property in physical and virtual manifestations- will persist too. The assumption that publishers and librarians were outmoded floating around at the beginning of the 1990s if it has not disappeared, it is certainly discredited. However, informal publishers and distributors are now on the scene and are unlikely to disappear. It is a more complicated world.

Three: Yet another fact of the Internet age of scholarly communication is that there is more empirical data available concerning the behaviors of readers and authors.

Four: One might be tempted to assert that the cost of publishing and library functions has by some measures gone down in the Internet age, but really our efficiency and productivity have increased greater than the costs have risen. First copy costs are arguably unchanged, but the cost of distribution to a vast and world-wide audience is much reduced.

Five: A final fact is that those connected to the Internet, despite problems with bandwidth and access in some parts of the world, form a new and much larger marketplace than ever before for ideas and the expressions of ideas. This new marketplace consists of a great many subordinate markets for the exchange of ideas and value, crossing all the usual boundaries. And denial of access to the new marketplace is a definition of the digital divide.

My selective list of possibilities for presses and libraries to collaborate is presented in order from the mundane to the celestial to the fantastic. Please let me start at ground level and work up. And please remember that the combination of these possibilities could be significant too.

Because we get more data about what readers are reading and even how they are reading as well as more and better data about what authors are doing, including citing other works and becoming multi-media creators, it seems to me that university presses might get some new marketplace indicators helping them make choices, to stimulate authors, to modify publishing programs at various levels, to improve the content as well as the forms and functions of scholarly communication. To provide an example from life, some publishers track the number of readers of articles in order to increase the number of articles on specific subjects; this might be called responsive intensifying by subject. A responsible university press would not want only to focus on what readers are known to prefer at a moment in time, because they would miss opportunities to present brand new topics. On the other hand, insight into the marketplace of ideas is always a good thing. I have often thought that we could decrease the amount of articles published in some fields by offering good enough pre-print services. A crafty publisher would want to know which authors and topics in the pre-print services were more heavily read than others in order to attract overlooked authors and to identify new topics of interest. In our present system, a cascade of publishing opportunities, in principle practically everything can get published. We could use more discrimination as publishers and librarians to get more efficient. For presses and libraries, knowing more and more about our readers, as communities or populations as well as individuals, will enable better customizing of information, a good thing, as well as more accurate targeting for more crass purposes, in some cases not so good a thing.

In the information chaos, university presses and university libraries perform valuable roles in selecting and validating information and knowledge. One wonders whether there is a possibility for these two communities working more explicitly together to identify new works in existing genres, say reference books and their digital equivalents, or to build together virtual versions of existing genres. In this cluster of possibilities is the 
adoption by presses and libraries of grass roots efforts, such as the on-line encyclopedia of philosophy underway at Stanford, when finally the initiators of such efforts realize that their child has reached adolescence and needs professional assistance to continue. Another possibility is that of the community of presses and university libraries collaborating to develop and maintain guides to the sources of information and knowledge in a host of specialized topics. It really would be very helpful to have editorial AND bibliographic expertise applied to fields such as Islamic studies, fisheries, global warming, and generative techniques in Renaissance poetry. Even in the application of navigation and discovery devices to the vast information chaos of the Internet, presses and libraries working together with software engineers could improve the ways these tools work on demand by individual academic readers.

Aggregators of digital information from a variety of publishers and on all available subjects are now attempting to make a commercial success of a shotgun approach to serving academic communities. There is beginning to be a backlash to this approach by both publishers and consumers of information. Publishers are becoming wary of the aggregator as a necessary intermediary to potential customers. Perhaps aggregators are not necessary at all from the perspective of publishers. And publishers are realizing that by better controlling their own information environment, even in active cooperation with competitor publishers, better services and enhanced opportunities for research in the literatures of academic disciplines are made possible. Institutional consumers, libraries, are wary of aggregators because they have learned that the aggregators are charging them for a lot of information that may never be used by their specialized communities of readers. It will be instructive to see how the aggregators fare over the long haul.

Specialized aggregations of content, on the other hand, appear to be useful enterprises to pursue. One involving the Columbia University Press and the Columbia University Libraries is their growing array of working papers, conference reports, government and NGO documents, and news reports in the area of international affairs. Its title is Columbia International Affairs Online and its acronym, especially suitable for this setting, is CIAO. This effort involves people from the university press in editorial roles and people from the university libraries in selecting and organizing material for inclusion in the site. There are 65,000 pages of documents on the site and it grows by 3,000 pages each month. It is heavily used by a growing list of customers and has earned critical acclaim. Moreover, it is a prime example of exactly the sort of collaborative work by university press and library staff which I find to be such a hopeful sign for the future of presses and libraries.

Knowledge environments are highly specialized digital libraries. They are libraries in the sense of providing collections of information along with information services, including the support of direct communication among those using the knowledge environments. These sites bring together journal articles, encyclopedia entries, specialized dictionaries, monographs and other content published by many different organizations and this content is kept up-to-date on a daily basis. The editors, librarians, and information technologists who oversee knowledge environments commission new content usually in the form of review and perspective articles for the sites and they annotate incoming content from the various sources, providing highlights to that which they believe to be the most significant. In addition, these knowledge environments may include protocols and methods, recipes for performing lab research, social scientific inquiries and surveys, and even possibly humanistic research. Knowledge environments include novel means for navigating such complex information spaces and feature personalization functions for subscribers such as keyword, concept, and citation alerting as well as individual collections of links in the site in reader defined virtual folders. Moderated and wide open threaded conversations 
encourage subscribers to communicate among themselves while organizational and personal biographical sections encourage members to get to know one another. There are several such knowledge environments in operation now on such diverse scientific subjects as cellular signal transduction, cancer research and treatment, bone and mineral research, and cardiology. More are in development. Two of particular interest because they treat complex subjects involving scientific and social scientific aspects of topics are aimed at communities of knowledge workers in the sciences of ageing and aqua-culture. It is the combination of editorial skill and judgment applied by publishing personnel, information science and knowledge management by librarians, and technical skills from programmers, database designers, and graphical user interface specialists which make these narrow but very deep digital libraries possible. These too are examples of how the new network technologies and the drive to address the needs of particular groups of users bring together publishing people, librarians, and technologists in new collaborations.

We will soon see new Internet genres promoting research and communication as well as education in areas of: public policy, law, organizational and operations research. These new genres will resemble knowledge environments in many ways, but will permit public and private research and communications functions to occur in parallel. The private elements will be devoted either to defined sub-groups of users, say government employees, or to self-defined groups, perhaps brought together by a specific legal case or public policy interest.

In the area of art, art history, and museums, there are embryonic collections of art and photographic images being assembled to make access to these images easier for use in teaching. The museum directors behind these efforts also want to advertise their exhibits and satisfy their missions of enriching the lives of citizens, many of them so distant from an exhibit that they might never visit it. These directors are also quite interested in protecting their rights as owners of the original images against exploitation of those rights. As image retrieval engines get more sophisticated, we are going to see new connections made by topic, by artists, ateliers, and schools of art, by iconic elements, by technique and by provenance. Such sites as these are made possible by museums as publishers and often their library staffs are deeply involved in aspects of the sites.

Returning once again to the sciences, there is a new school of specialists assembling because of startling new possibilities for research and development of therapies arising from the vast amount of data about humans stored in many different forms and places. This new school at Stanford is tentatively labeled the computational life sciences. Members of the school come from a wide array of disciplines and professions. There are radiologists, surgeons, statisticians and applied mathematicians, electrical engineers and computer scientists, roboticists, librarians and information technologists, historians of science, pharmacologists, geneticists and genomicists, cell and molecular biologists - just to name a few. This group of over 80 people was drawn together by the realization that the advances each made on the basis either of massive data bases or very high end engineering were just precursors to more spectacular findings and therapies. Data warehousing and data mining will thus become significant aspects of this new school of research. Indeed, this group is now assembling even more data on individual humans as well as on populations, some of it longitudinal. Key parts of the data brought together for research is published material; finding new ways to make connections from the publications to the datasets is an important task; so publishers are involved here too. Some of the research is devoted to new findings of the operations of the human genome and other work is advancing micro-surgical techniques for very precise and low invasive surgery. We are just starting this work at Stanford, but we know of similar groups forming at Columbia, Harvard and Chicago. 
These few examples of new sorts of scholarly communication or, in the case of the last example, a new discipline needing that communication, are merely indicators of the array of opportunities and challenges facing publishers, including university presses, and research librarians. What each example illustrates is the requirement for focus on the needs, aspirations, practices, and qualities of specific knowledge workers as consumers and producers of content. This requirement for focus also suggests that niche publishing and specialized librarianship will be fruitful areas for professionals in each trade. And it should alert us to the new possibilities for collaboration between presses and libraries.

Perhaps in a few decades or even sooner, the analogy of tinkers and tailors which now so aptly describes the lack of effective collaboration but as well the complementarity between university presses and libraries will be replaced by a new analogy, that of Florence and the new Renaissance - Firenze e il rinascimento nuovo. 


\title{
The Self-Archiving Initiative
}

\author{
Stevan Harnad \\ Professor of Cognitive Science \\ Intelligence/Agents/Multimedia Group \\ Department of Electronics and Computer Science \\ University of Southampton \\ Highfield, Southampton, UK \\ E-mail address: harnad@cogsci.soton.ac.uk \\ http://www.cogsci.soton.ac.uk/ harnad/
}

\begin{abstract}
Researchers publish their findings in order to make an impact on research, not to sell their words. Access-tolls immediately by self-archiving theme on-line in their own institution's Eprint Archives. Free eprints.org software creates Archives compliant with the Open Archives Initiative metadata-tagging Protocol OAI 1.0. These distributed institutional Archives are interoperable and can hence be harvested into global "virtual" archives, citation-linked and freely navigable by all. This should enhance research productivity and impact as well as providing powerful new ways of monitoring and measuring it.
\end{abstract}

Unlike the authors of books and magazine articles, who write their texts for royalty or fee income, the authors of refereed journal articles write them only for "research impact", which means for their effects on research and researchers. In order to reach researchers and to have an effect on their research (so the latter can use the findings in their own work), these refereed journal articles have to be accessible to their potential users. Hence, the idea that access to them should be toll-gated in any way makes as much sense as tollgated access to commercial advertisements.

In other words, unlike the author royalty/fee-based literature, which constitutes the vast majority of the printed word, whether on-paper or on-line, this special, tiny, anomalous literature -refereed journal articles- is an author give-away: Its authors have never sought nor benefited in any way from the fact that access-tolls had to be paid to read their papers (in the form of individual and institutional subscriptions [S], and lately, for the on-line version, site-licenses $[\mathrm{L}]$ or pay-per-view $[\mathrm{P}]$ ). On the contrary, those $\mathrm{S} / \mathrm{L} / \mathrm{P}$ access-barriers represent, and always did represent, impact-barriers for these authors, whose careers (promotion, tenure, funding, prizes) depend largely on the size of the research impact of their work.

How big are the impact barriers? There are currently at least $20 \mathrm{~K}$ refereed journals across all fields of science and scholarship (this estimate could be as much as an order of magnitude too low), in which at least $2000 \mathrm{~K}$ (probably many more) refereed articles appear annually. If we calculate the amount that all the institutions on the planet who can afford the $\mathrm{S} / \mathrm{L} / \mathrm{P}$ tolls (individual subscriptions are negligible in this reckoning) collectively pay for just one of those $2000 \mathrm{~K}$ refereed papers, it averages about $\$ 2,000$ per paper. 
That amount is what just those institutions who can and do pay for access pay. In exchange, that particular Give-Away paper is accessible to researchers at those, and only those, institutions. (Don't think of everyone else looking at it on the shelf of a public library: That does not scale up to the sort of free and full access that is possible in principle, especially online).

The research libraries of the world can be divided into the (minority) Harvards and the (majority) Have-Nots. It is obvious how the Have-Nots (and they prevail everywhere, not just in the Developing World) would benefit from free access to the entire refereed literature, for without it their meager S/L/P budgets can afford only a pitifully small portion of it. But not even Harvard can afford access to anywhere near all of it (http://fisher.lib.virginia.edu/newarl/index.html). So the fact is that most of the annual $2000 \mathrm{~K}+$ refereed articles are currently inaccessible to most of the researchers on the planet. For the authors of those articles, this means that much of their potential impact (and actual access) is lost. And this curtailed research impact and access is what the $\$ 2000$ per article currently being spent by the planet in $\mathrm{S} / \mathrm{L} / \mathrm{P}$ tolls is buying it.

This is the way things were, and the way they had to be, in the Gutenberg Galaxy, in which publishing as print-on-paper was the only way to make refereed research accessible to other researchers, and the sizeable costs of printing and distribution had to be recovered through access-tolls if the research was to have any impact at all. But we have to remind ourselves that we are no longer in the Gutenberg Galaxy. This new state of affairs has much less positive consequences for the majoritarian, Non-Give-Away literature (books, magazine articles), written for royalties and fees, and now at risk of digital piracy, Napster/Gnutella-style. But for the Give-Away literature, the PostGutenberg Galaxy is a godsend, for it at last makes it possible to eliminate all access/impact-barriers to refereed research.

It is not that all costs have vanished. Note that we are speaking about refereed research, not about some vast digital Vanity Press (for otherwise the analogy between researchers and advertisers would become a homology). Although the Gutenberg costs of printing and distribution (and even the costs of their on-line successors, such as journal publishers' PDF page-images) are no longer necessary ones, the cost of the quality-control and certification $(\mathrm{QC} / \mathrm{C})$ that differentiates the refereed literature from an unfiltered, anarchic, pot-luck Vanity Press still needs to be paid. Paper and PDF have become mere options, purchasable by those who want and can afford them; the refereeing, however, is and continues to be a medium-independent essential for scholarly and scientific research.

But what is refereeing, and what does it cost? Refereeing (also called "peer review") is the system of evaluation and feedback by which expert researchers assure the quality of one another's research findings. The referees' services, like the authors' research papers, are Give-Aways, so that is not where the remaining expense lies. It is the implementation of the refereeing procedures that necessarily entails some cost. But how much? How much does it cost for submitted manuscripts to be archived on a website, for an expert Editor to pick expert referees, email them the website of the submission, receive their emailed referee reports, email the author the reports plus an editorial disposition letter indicating what revision needs to be done to make the manuscript acceptable, and to repeat the process (if necessary) until the manuscript is accepted or rejected?

There is general agreement that the upper limit on the cost of implementing refereeing is not more than $\$ 500$ per accepted article (http://documents.cern.ch/archive/electronic/other/agenda/a01193/a01193s5t11/transparencies/) but even that figure almost certainly has needless Gutenberg costs wrapped into it (e.g., the creation of the publisher's PDF version). The true figure for peer-review implementation alone is probably much closer to $\$ 200$ per article or even lower. In other 
words, QC/C costs account for only $10 \%$ of what the planet currently spends per article in those $\mathrm{S} / \mathrm{L} / \mathrm{P}$ tolls that restrict access to this Give-Away literature to only that minority of researchers who do not happen to be at a Have-Not institution for that particular article.

Is there any way to remedy this situation, in which this Give-Away PostGutenberg literature is being needlessly held hostage to obsolete Gutenberg costs and cost-recovery methods? First, let us note that it is not simply a matter of lowering the S/L/P access barriers. Even if the S/L/P tolls for all $20 \mathrm{~K}$ refereed journals were slashed by $90 \%$, that would still leave most researchers on the planet unable to access most of this author Give-Away research. No, there is only one solution, and it is an inevitable one: The refereed research literature must be freed, for everyone, everywhere, forever, online. And the irreducible $10 \% \mathrm{QC} / \mathrm{C}$ costs must no longer be paid for by the reader-institution, in the form of S/L/P tolls (reduced to 10\%), with their attendant impact/access-barriers. Instead, they must be paid for as QC/C service costs by the author-institution, per paper published by its researchers, funded out of $10 \%$ of the institution's annual windfall S/L/P savings.

How to get there from here? Journal publishers will certainly not scale down to becoming only providers of the essential QC/C service (plus whatever add-on options there is still a market for) of their own accord: No one would. Nor can libraries, already weighed down by their escalating serials crisis, redirect any of their so far nonexistent windfall savings to any other purpose. Nor can authors be expected to sacrifice submitting their research to their established high-quality, high-impact journals, submitting it instead to new, alternative journals, with no track records, authorships, or niches, just because those journals happen to be prepared to provide $\mathrm{QC} / \mathrm{C}$ alone right now. Journal niches are largely saturated already, and tenure/promotion/funding/prizes are far more important to researchers now then any potential longterm benefits (how soon?) from making risky sacrifices now.

There is a way, however, that researchers can have their cake and eat it too, right now. The entire refereed journal literature can be freed, virtually overnight, without authors having to give up their established refereed journals. The way has already been tried and proven to work by a portion of the Physics community. They have been publicly selfarchiving their research papers online -both before and after refereeing, i.e., both preprints and postprints- since 1991. It is very important to note that this Physics "Eprint" Archive (http://www.arxiv.org) includes, and has always included, the refereed postprints too, for it has often been confusingly and incorrectly described as a "Preprint Archive" (with the implication that it is merely a Vanity Press for unrefereed papers). Preprint and postprint are merely successive embryological stages of a refereed, published journal article.

The Physics Eprint Archive (currently 150K papers in all) has been growing steadily. The annual number of new papers self-archived therein is now about $30 \mathrm{~K}$ and increasing by about $3.5 \mathrm{~K}$ per year. The archive, with its 14 mirror-sites world-wide, gets about $175 \mathrm{~K}$ user "hits" per weekday at its US site alone. So there is no doubt that self-archiving can be done, and that when papers are thus made freely accessible online, they are indeed accessed, very heavily.

The problem is that although the Physicists have shown the way to free the refereed research literature, other disciplines have been slow to realize that it will work for them too. They have assumed that there must be something unique about Physics, and that the self-archiving strategy is pertinent only to Physics. This misapprehension has been encouraged by the (incorrect) impression already mentioned that it is only the unrefereed literature that the Physicists have freed online, and that doing so somehow puts at risk or compromises QC/C. Yet the fact is that absolutely nothing has changed with regard to peer review in Physics! The very same authors who self-archive continue to submit all 
their papers to their established refereed journals of choice, just as they always did, and virtually all the papers in the Archive appear in refereed journals about 12 months after journal submission. Nothing has changed except that a growing portion of the refereed literature in Physics is at last accessible free for all online (including earlier embryological stages that were not previously accessible at all).

The second problem, after the fact that the other disciplines have been so slow in following the lead of the physicists, is the fact that even in Physics, self-archiving is growing far too slowly: At the present linear growth rate, it will be another decade before the entire Physics literature is online and free. Something else is needed, both to accelerate the rate of self-archiving in Physics, and to extend the practise to all the other disciplines.

And that something else has now arrived. The reason the "subversive proposal" to free the refereed literature through author self-archiving fell largely on deaf ears in the early 90's (Harnad 1995) was that self-archiving in an anonymous FTP archive or a Web HomePage would have freed the literature only in principle. In practise, all those scattered online papers, their locations, identities and formats varying arbitrarily, would be unsearchable, unnavigable, irretrievable, and hence unusable (unless one happened to know where a particular paper was in advance). Yet centralized archiving, even when made available to other disciplines (e.g. http://cogprints.soton.ac.uk) has not been catching on fast enough either ( $\mathrm{Cog}$ Prints has taken 3 years to reach $1 \mathrm{~K}$ articles).

What was needed was something that would make the fruits of distributed, institutionbased self-archiving equivalent to those of centralized self-archiving, and the key to that was to introduce and agree upon metadata-tagging standards that would make the contents of all the distributed archives interoperable, hence harvestable into one global "virtual" archive, all the papers searchable and retrievable by everyone for free, without having to know in advance where they happened to be individually archived, or in what form.

The Open Archives Initiative (OAI) (http://www.openarchives.org) has provided the meta-data tagging standards and a registry for all OAI-compliant Eprint Archives, and the Self-Archiving Initiative (http://www.eprints.org)has provided the (free) software for creating OAI-compliant Eprint Archives, interoperable with all other Open Archives, ready to be registered and for their contents to be harvested into searchable global archives (http://cite-base.ecs.soton.ac.uk/cgi-bin/search/).

Distributed Institution-based self-archiving is the natural way to generalize the practise of self-archiving across disciplines and institutions. It is not only the author who benefits from research impact. The reason promotion and tenure are contingent on research impact is that funding is contingent on it too. Hence institutional funding overheads and prestige are as much the beneficiaries of the freeing of their researchers' refereed research from any needless impact-barriers as individual researchers (and research itself) are. "Publish or perish" has always been an oversimplified slogan. What is meant by it is neither unrefereed (vanity-press) nor unread/uncited (impactless) publication. Written out in realistic longhand, the institutional slogan would be "Maximize your refereed research impact to maximize your (and our) rewards from it.

So researchers' institutions are not only natural allies in freeing their researchers' refereed research from any unnecessary impact-barriers, they are in a position to lead and speed the way (by providing and supporting the institutional archives and encouraging, indeed mandating their filling with their researchers' refereed papers). No such collective self-interest unifies or propels centralized, discipline-based self-archiving. Nor do the institutional benefits of distributed self-archiving stop with eliminating the impact-barriers to their own institutional researchers' research: Eliminating, for their own researchers, the access-barriers to the research of others, at other institutions, is another way of increasing their own research productivity and impact. 
And that brings us to a third potential institution-level benefit weighing in for distributed institution-based self-archiving: the prospect of a solution to the spiraling serials budget crisis: The likelihood of eventually reducing the institutional library's annual serials expenditures to $10 \%$ (simply by eventually redirecting that proportion of the annual windfall $100 \%$ savings to covering the journal peer review implementation costs for their own researchers' refereed publications) is not only an added incentive for hastening the transition by facilitating institutional self-archiving. It also provides allies from the institutional library, who can (1) help researchers in the first-wave of self-archiving (selfarchiving for them by proxy if need be), (2) maintain and preserve the institutional refereed Eprint Archives as an outgoing collection for external use, in place of the old incoming collection, acquired through S/L/P, for internal use. (3) Institutional library consortial power can also be used to provide leveraged support during the transition for journal publishers who commit themselves to a timetable of down-sizing to becoming pure $\mathrm{QC} / \mathrm{C}$ service providers.

Here is a summary of this transitional scenario, explicitly separating the parts that are certain to produce the promised results from those that are in any way hypothetical, or conditional on what happens next:

(1) Once all the $2000 \mathrm{~K}+$ annual refereed journal articles are self-archived by their authors in their institution's registered, OAI-compliant Eprint Archives, this literature is de facto freed from all access- and impact-barriers online. The self-archiving could be done virtually overnight, and the day after, it would cease to be true that most of this giveaway research is inaccessible online to most of the researchers on the planet. That is guaranteed.

(2) It is conceivable that that will be the end of it. The refereed literature will be free online for those who want it and cannot get it any other way, but those who can afford to get it the old way (via $\mathrm{S} / \mathrm{L} / \mathrm{P}$ ) will continue to do so. In that case, the access/impact problem will be solved, but the serials crisis will not; it will simply become a much less urgent matter.

(3) But if, contrary to (2), when the refereed literature is accessible online for free, users prefer to use the free version (as so many physicists already do), then journal S/L/P revenues may shrink and institutional S/L/P savings may grow. Journals will then have to begin to scale down to providing only the essentials (the QC/C service), with the rest (onpaper version, on-line PDF version, other "added values") sold only as an option, as long as there is still a market for it. Those journals that agree to scale down to providing only the essentials ( $\mathrm{QC} / \mathrm{C}$ at $\sim \$ 200$ per accepted final draft) can make a leveraged transition agreement with a library consortium that will prop them up out of reader-institution-end $\mathrm{S} / \mathrm{L} / \mathrm{P}$ while they downsize, but according to a schedule with an agreed time by which they must have switched to author-institution-end QC/C cost-recovery $(\sim 200)$ per accepted paper. The titles (editorial boards, refereeships, authorships) of journals whose publishers are not interested in continuing operations in such a downsized niche can migrate to new QC/C-only publishers who are, and who thereby instantly inherit an established journal.

Note that peer review itself is never compromised, sacrificed, or put at risk in this scenario; nor do authors have to give up, even temporarily, submitting to their established journal of choice. All they have to do is self-archive their preprints and postprints in their institutional Eprint Archives.

Are copyright restrictions any obstacle to self-archiving? Not at all. Pre-refereeing preprints can be self-archived without any restriction. For the post-refereeing, accepted final draft, the author can first try to modify the copyright agreement, transferring to the publisher all rights to give away, lease or sell the text, on-paper or on-line, in perpetuo, 
but retaining only the author's right to give it away online for free by self-archiving it. Publishers will not put that in their copyright agreements of their own accord in advance (although some already do, e.g., the American Physical Society: ftp://aps.org/pub/ jrnls/copy_trnsfr.asc), but many will accept it if the author asks. For those papers, simply self-archive the refereed postprint alongside the pre-refereeing preprint(s). For those publishers who refuse to publish the paper unless all rights are transfered: Sign the restrictive agreement, and self-archive a linked "corrigenda" file listing for the user what changes have to be made in the preprint to make it equivalent to the postprint (http://www.cogsci.soton.ac.uk/ harnad/Tp/resolution.htm\#Harnad/Oppenheim)

\section{References}

Harnad S. 1990 Scholarly Skywriting and the Prepublication Continuum of Scientific Inquiry: Psychological Science 1: 342-343 (reprinted in Current Contents 45: 9-13, November 11 1991).

[http://cogsci.soton.ac.uk/ harnad/Papers/Harnad/harnad90. skywriting.html].

Harnad S. 1991 Post-Gutenberg Galaxy: The fourth revolution in the means of production of knowledge: Public-Access Computer Systems Review 2 (1): 39-53.

[http://cogsci.soton.ac.uk/harnad/Papers/Harnad/harnad91.postgutenberg.html].

Harnad S. 1995 Universal FTP Archives for Esoteric Science and Scholarship: A Subversive Proposal: Ann Okerson \& James O’Donnell (Eds.) Scholarly Journals at the Crossroads; A Subversive Proposal for Electronic Publishing. Washington, DC., Association of Research Libraries, June 1995. [http://www.arl.org/scomm/subversive/toc.html].

Harnad S. 1998/2000 The invisible hand of peer review. Nature [online] (5 Nov. 1998) [http://helix.nature.com/webmatters/invisible/invisible.html]. Longer version in Exploit Interactive 5 (2000): [http://www.exploit-lib.org/issue5/peer-review/ http://www.cogsci.soton.ac.uk/ harnad/nature2.html].

Harnad S. 2000 E-Knowledge: Freeing the Refereed Journal Corpus Online. Computer Law \& Security Report 16(2) 78-87. [Rebuttal to Bloom Editorial in Science and Relman Editorial in New England Journal of Medicine] [http://www.cogsci.soton.ac.uk/ $\sim$ harnad/Papers/Harnad/harnad00.scinejm.htm [http://www.sciencemag.org/cgi/eletters /285/5425/197\#EL12].

Harnad S. 2000 Ingelfinger Over-Ruled: The Role of the Web in the Future of Refereed Medical Journal Publishing: The Lancet Perspectives 256 (December Supplement): s16. [http://www.cogsci.soton.ac.uk/ harnad/Papers/Harnad/harnad00.lancet.htm].

Harnad S. 2001 For Whom the Gate Tolls? How and Why to Free the Refereed Research Literature Online Through Author/Institution Self-Archiving, Now.

[http://www.cogsci.soton.ac.uk/ harnad/Tp/resolution.htm].

Harnad S., Carr L. \& Brody T. 2001 How and Why To Free All Refereed Research From Access- and Impact-Barriers Online, Now.

[http://www.cogsci.soton.ac.uk/ harnad/Tp/science.htm].

Harnad S., Varian H. \& Parks R. 2000 Academic publishing in the online era: What Will Be For-Fee And What Will Be For-Free? Culture Machine 2 (Online Journal) [http://www.cogsci.soton.ac.uk/ harnad/Temp/Varian/new1.htm].

[http://culturemachine.tees.ac.uk/frm_f1.htm].

Odlyzko A.M. 1998 The economics of electronic journals. In: Ekman R. and Quandt, R. (Eds) Technology and Scholarly Communication. Univ. Calif. Press, 1998. [http://www.research.att.com/ amo/doc/complete.html]. 
Odlyzko A.M. 1999a Competition and cooperation: Libraries and publishers in the transition to electronic scholarly journals, A. M. Odlyzko. Journal of Electronic Publishing 4(4) (June 1999) and in J. Scholarly Publishing 30(4) (July 1999), pp. 163-185. The definitive version to appear in The Transition from Paper: A Vision of Scientific Communication in 2020, S. Berry and A. Moffat, eds., Springer, 2000. [http://www.press.umich.edu/jep/04-04/odlyzko0404.html].

Odlyzko, A.M. 1999b The rapid evolution of scholarly communication, to appear in the proceedings of the 1999 PEAK conference.

[http://www.research.att.com/ amo/doc/rapid.evolution.pdf]. 



\title{
The Roquade Project. The Library as a Catalyst of Change in Scholary Publishing
}

\author{
Bas Savenije \\ University Librarian \\ Utrecht University, NL \\ E-mail address: b.savenije@library.uu.nl
}

\begin{abstract}
Due to a number of problems the traditional scientific journal has become an obstacle for efficient scientific communication. Several initiatives have been started to realise other ways of academic publishing, using modern information technology. One of these initiatives is the Roquade project, initiated by the libraries of the Dutch universities of Utrecht and Delft. It aims at creating an infrastructure that conglomerates the swiftness of publication which hitherto could only be realised by grey publishing, with quality judgment without the serious delay of the traditional review procedures. Roquade offers a wide number of facilities to a broad audience, based on a common organisational and technical infrastructure. The extraordinary aspect of the Roquade project is marked by the fact that this variety of possibilities constitutes an expeditious way for gradually changing the publication behaviour of scientists. The project has been started by two university libraries, which consider it as their mission to cover the entire information chain. By reallocation of funds and training of library staff, traditional library functions are gradually replaced by innovated library functions or entire new functions related to the extended mission of the library. The presentation describes the Roquade project, its philosophy, organisation and first results, but also the rationale behind the library's involvement in scholarly publishing.
\end{abstract}

\section{Introduction}

Developments in information technology have consequences for the processes of scientific publishing and scientific communication. It is to be expected that these processes will change drastically. This paper analyses these changes and addresses the question whether a university library might have a future role in electronic publishing. It also describes the Roquade project, initiated by the Dutch university libraries of Utrecht and Delft, which aims at creating an infrastructure for electronic publishing. Finally, it deals with the recent initiative Signal Hill aimed at setting up a community of practice for academic epublishing initiatives.

The structure of the paper is as follows: section 2 describes a number of important trends relevant to the future of scholarly publishing. Section 3 is concerned with the role of a university library in publishing. In section 4 is explained how innovative projects like Roquade are started and embedded in a library organisation. Section 5 describes the 
Roquade project, section 6 Signal Hill, a recent initiative to stimulate interaction and cooperation between academic e-press initiatives. Finally, section 7 draws some general conclusions.

\section{Some important trends}

\section{The information chain}

The role of the library and as well that of the publisher are often described as links in the information chain. There are currently various problems connected to the information chain. The chain stretches from information production to consumption. Traditionally, the various stages have been strictly distinguished in terms of the chain's main functions, i.e. information production, distribution, acquisition and consumption. Clearly identified parties, such as the publisher, the subscription agent and the library, carried out these activities.

This chain is usually represented schematically. The traditional representation was fairly simple. Lately, however, it seems to have become more complex due to the pressure of an ever-expanding information stream and as a result of various technological developments. It has even been suggested that the chain is about to explode. However, this metaphor of an exploding chain or cycle is not accurate. The chain is not about to burst at all. In fact, it is the amount of information that is growing explosively, and this is due to the way the information is being transferred. Yet, all the functions in the traditional information chain, i.e. production, distribution, acquisition and knowledge consumption, can be carried out at a so-called integrated work site. Within this context, the image of an imploding chain would be more appropriate, since the system is starting to shrink due to the pressure exerted by the environment.

The configuration of functions within the information chain has been subjected to change, whilst within this changing constellation, all the parties involved are struggling to determine their positions. For instance, all kinds of arrangements have been made between universities and publishers to facilitate the harmonious exploration of their new roles. However, it is important for all parties not to remain fixed in the traditional chain. In view of the developments taking place in the field of information provision, it is clearly necessary to experiment creatively and innovatively with new roles instead of adhering to traditional patterns. All forceful attempts to preserve the old structure will not only impede development as such, but will also expose the structure to the increasing danger of arbitrary disintegration.

\section{The serials crisis}

The philosophy behind the familiar adage 'publish or perish' has resulted in a noticeable growth in the number of scientific papers. As a consequence of this growth, considerable problems and bottlenecks are impeding the traditional system of scholarly publishing (see also Savenije 1997).

1. The system is sluggish: it takes at least six months, sometimes up to a year and a half, for a submitted paper to actually be published.

2. There is increasing doubt as to the system's reliability, particularly since reviewers could use their prior knowledge to take advantage.

3. The system is becoming unaffordable due to considerable price increases that sometimes exceed the general price index. These price rises lead to subscriptions cancella- 
tions which in turn stimulate new increases, eventually evolving into almost monopoly-like situations.

Furthermore, we must be aware of the fact that, in addition to these price increases, universities have to pay for these publications more than once, because they also fund:

- the salaries of university staff members who write the articles and papers;

- the salary of the reviewer who, at the request of the publisher, judges whether the articles are suitable for publication;

- the publication's purchase price;

- the archiving of the publications.

A number of problems might be solved with the help of information technology. However, this calls for an innovative attitude towards publishing. Presently, it can be observed that traditional commercial publishers are reluctant to experiment with innovation.

\section{The role of the university library}

From the trends described above we can conclude that the academic community should endeavour to keep the processes of scholarly publishing in its own hands. Furthermore, universities should use the possibilities offered by information and communication technology to experiment with new publishing models in order to incorporate and emphasise the essential aspect of communication in scholarly publishing.

Traditionally, the university library is responsible for selecting, collecting, preserving and providing access to information. The publisher's distinctive tasks in the information chain are distribution and quality certification. The distribution of electronic documents is a process that shares many similarities with the library's function of providing access to information, particularly when this task is combined with storing and preserving.

Regarding quality certification, the peer review is carried out by academics and organised by publishers. There are two obvious organisational alternatives to this:

- the process is organised by publishers, who act not as owners of the information but as service providers hired by the university;

- the process is organised by libraries. One of the advantages of this alternative is the possibility of direct contacts between the library and the scholars and their personal networks. When the publishing processes and the publishing output are electronic, the support processes that enable scholars to publish their results share many similarities with the digital library tasks. If the academic community wishes to take control of scholarly publishing, an organisation within the university will have to organise the support processes and the back office functions that facilitate scientific publication. The university library is the natural candidate, provided that it is able to make the transition from a traditional to an electronic library.

Two aspects of electronic publishing support can be distinguished (Savenije \& Grygierczyk 2000).

The first aspect involves tasks carried out for the benefit of the 'parent institution' of the library, the university in which the library is embedded. The priorities are to store, preserve and providing access to the university's scientific output (e.g. its own publications). These tasks are very close to traditional library activities. The essential objective of these tasks is to create a digital archive of the scientific output of the university, to preserve this information and make it accessible to the academic community, within as well as outside the university.

The second aspect is more discipline-oriented and takes into account that scientific publishing and communication are not limited to one institution. This is the role that 
emphasises the necessity of new publishing models that enhance scientific communication, accelerate the exchange of research results and organise open discussions in knowledge domains. The facilitating role of the library in this context is mainly performed by organising the processes needed for electronic publishing. Researchers can put their papers on the website of their institute or initiate electronic journals, as well as experiment with publication servers and new peer review models. The initiative for these activities will come from researchers within faculties, from research institutes and learned societies, but they will be able to make use of the back office support provided by the library, including the organisational and technological capacities.

\section{The innovation policy at Utrecht University Library}

Due to the developments in information technology a library needs to reconsider its services, but also its strategic position in relation to its environment: its "parent institution", and the other partners in the information chain. This inevitably leads to the need of rigorous innovation.

With respect to the question of how this innovative process needs to be organised, a number of factors is of importance.

First, the innovation process requires a certain amount of creativity.

Secondly, the innovation process is no simple matter due to a number of factors:

- The complex organisation of the university library: the relationship with faculty policies often results in organising several branch libraries, which are part of one or several faculty structures.

- The many different categories of library services involved: this implies a comprehensive approach in which a large number of people must participate.

- The rapid technological developments: changes in standards may interfere with the plans concerning the process of change; therefore, a flexible approach is needed.

These factors underline the importance of the special attention the library management must pay to the innovation process.

\section{Project organisation}

The best manner to realise innovation is to introduce a temporary separate organisation assigned for the sole purpose of the innovation project. Or, to be more specific, a project organisation with independent responsibilities, a special project manager, and a separate budget.

As a matter of fact, this is the only way to observe the deadlines and monitor results in a detailed fashion.

When looking more closely at the innovation process, we notice that it actually consists of a large number of subsidiary projects, each with discriminate objectives, different time spans and budgets, as well as various project leaders.

Of vital importance to the project organisation are:

- a general project manager,

- a few project co-ordinators,

- one or more steering groups,

- a number of work groups.

The tasks of the overall project manager comprise:

- the co-ordination of the project as a whole,

- guiding the project co-ordinators and the various project leaders, 
- monitoring the progress of the subsidiary projects,

- taking care of external contacts,

- defining and initiating new projects,

- preparing requests for external grants and subventions.

The general project manager is supported by a few project co-ordinators appointed to support a well-defined range of subsidiary projects, e.g. for projects having an infrastructural nature. Furthermore, every separate subsidiary project needs to have its own project leader.

The general project manager is not to belong to the staff of any library department and should report directly to the library head management. The project co-ordinators are appointed to work exclusively for the project. The remaining participants should only be partly relieved from their regular activities and must combine their innovation process contributions with their regular job.

The transfer of results to the regular organisation

During completion of each subsidiary project, the results for the regular organisation should be clearly determined. As to the transfer of results, we can observe the following possibilities:

- The project in question does not require a follow-up in the organisation. Either it has proved to be unsuccessful or, considered from a strategic point of view, it is advisable not to continue the involved activity.

- The project result is of strategic importance, however as of yet does not prove ready for transfer to the regular organisation. In that case, it should be continued as a strategic innovation project supervised by a separate project organisation.

- The project in question is prolonged within the regular organisation, either as a project or as a new regular service. In this case, the consequences for the involved staff must be specified: the number of staff required as well as their (new) qualifications.

Actually, the transfer of results to the organisation as a new regular service is the best way to safeguard a project's results. In our approach this can be done as follows:

- involve regular library staff in the projects as well as in the description of the activities involved in a new or renewed service or product as a result of the project;

- the new activity should be implemented within one of the regular departments;

- if the staff members involved in the project are functioning satisfactorily, they are the first candidates for the new regular job that may evolve out of the new activity;

- guidance should be given to the implementation of the new activity by training programs for the staff involved, detailed descriptions of the procedures to be followed, evaluation and (if necessary) adjustment of these procedures.

This process has a number of advantages and disadvantages:

- because of the training of the staff involved, the process described takes almost twice as long, compared with the situation in which experienced project staff is hired; but

- there is more commitment of the participating staff to the results; and

- at the end of the project the activities can be taken over by the regular organisation immediately.

\section{Roquade}

Roquade is a joint project of Utrecht University Library, Delft University of Technology Library and the Netherlands Institute for Scientific Information Services of the Royal 
Netherlands Academy of Arts and Sciences. The objective of the project is to set up an infrastructure for electronic publishing. Its mission is to enhance scientific communication by offering scholars a wide variety of facilities and organisational structures, aimed at gradually changing the publishing habits of scientists. The raison d'être of this mission is the fact that a relatively large number of scientists still hesitate to use new publishing methods which would liberate them from the monopoly commercial publishers have with respect to the publication of new research results. Roquade presents the academic community with an infrastructure for electronic publishing, which facilitates a gradual revolution from traditional publishing to very innovative models. Close Cupertino with similar organisations constitutes a crucial factor in Roquade's mission.

Its main features are:

- Enhancing scholarly communication in its full diversity,

- Supplying knowledge, tools and people in the publishing area ,

- Not profit driven,

- A community of practice.

The point of departure is to safeguard the interests of the authors and the academic community. The project is directed towards all disciplines and concerns Dutch publications as well as international initiatives (Savenije \& Grygierczyk, 1999; see also http://www.roquade.nl).

\section{The main structure of the project}

To understand the main structure of the project it is necessary to know that Roquade comprises:

1. number of subsidiary projects with three different variants:
a. moderated pre-print service for grey literature
b. electronic substitution for traditional publishing
c. publication site with different peer review models

2. subsidiary project aimed at a technical and organisational infrastructure which is common for all variants

The first variant consists of a moderated pre-print service for grey literature. In this subsidiary project, grey literature is defined as publications, which are moderated but do not have a formal independent stamp of approval. In this case, dispatch prevails over time-consuming quality judgement.

The second variant can be described as electronic substitution for traditional publishing. Two categories of publications can be distinguished:

- dissertations and conference collections

- scientific journals

There are two subsidiary project types concerned with scientific journals:

- Publishing new and existing electronic journals: this involves providing support to editors who prefer the traditional form of electronic publishing, in which the electronic journal is an electronic 'copy' of the traditional paper journal.

- Co-publishing: setting up a series of digital publication services for publishers, for instance for electronic versions of existing paper journals.

It appears that most of the editors, who are interested in setting up an electronic journal, want to begin in a more or less traditional way, which involves mainly text and pictures. The concept of volumes and issues, typical of the paper journal, is maintained in the starting phase and, as in the case of traditional paper journals, peer review takes place before publication.

Gradually, a whole spectrum of additions and alterations are possible: 
- the concept of issues will become obsolete as work is presented as soon as it is ready for publication;

- research data can be added;

- communication and discussion facilities can be added;

- multimedia can be used to enrich the publication.

The third variant is a publication site with peer review. The main feature of this variant is the initiation of a series of pre and post-publication peer review processes, which makes as much use as possible of experience and knowledge gathered in traditional and digital academic publishing processes. Subsequently, the traditional process of peer reviewing is to be transformed into several new types, thereby preserving the traditional advantages as much as possible. Simultaneously, the benefits of the new medium and organisation should be exploited to the fullest extent. In this variant it is possible to achieve speed without omitting the time-consuming peer review process by delaying peer reviews until after publication. This variant also offers the possibility of experimenting with different forms of quality assessment.

These variants reveal the main objective of the Roquade project: a gradual transition from less innovative solutions (for instance a publication site for grey literature or a traditional electronic journal) to more innovative solutions (such as a publication site with post-publication peer review). Not is this objective feasible, in some cases, it is even natural.

\section{The phases of the project}

In the project the following phases can be distinguished.

1. The preparation phase (May 1999 - July 2000)

This phase comprises analysis and a user survey, assignment of project activities and setting up collaborations with similar initiatives and organisations.

2. The start-up phase (September 2000 - December 2001)

The main elements of the start-up phase are:

a. Setting up the infrastructure:

- the organisation and the production process

- availability and presentation

- archiving and storage

- security and authorisation

b. Pilots for each variant:

- moderated pre-print service for grey literature: facilitating the transition to variants with peer review

- launching new electronic journals

- co-publishing initiatives

- publication site with peer review for one or two selected disciplines

c. Financing

d. Evaluation

3. The development phase (January - July 2002)

The objective of this phase is to adapt and further expand the organisation, on the basis of the experience acquired in the pilot projects. The result should be a viable organisation with a good reputation.

4. Expansion phase (July - December 2002)

The objective of this phase is scale enlargement of:

- available material. This involves initiatives for increasing the number of journals, articles and grey literature on offer, increasing the number of disciplines and setting 
up new initiatives for collaboration.

- the audience, by means of focused PR activities.

At this moment the project is in the starting up phase, in which the infrastructure is built up and, contemporarily, tested out in satellite projects.

\section{The organisation}

The organisational structure of Roquade is as follows:

- A steering group, this consists of the library directors of the Dutch Royal Academy, and the Universities of Delft and Utrecht, plus a general project manager.

- A back office, consisting of a co-ordinator and a pool of specialists in Delft, Utrecht, Amsterdam for the maintenance and upgrade of self-publishing and administrative tools. - Front offices, which are franchisees of the Roquade brand (Delft, Utrecht, Amsterdam, and new front offices, together with a central contact and help-desk.

- Satellites, these are autonomous publishing initiatives and projects. They, in fact, are the clients of the front offices.

With this organisational structure Roquade hopes to prevent the presence of a strong intermediary organisation which sooner or later will have its own persistence as an important goal, thus becoming an obstacle for self-publishing initiatives.

\section{Current Roquade satellites}

The current Roquade satellites are:

Journals

- International Journal of Integrated Care

- International shipbuilding progress

- European Journal of Transport and Infrastructure Research

- Kronos - Journal of Cape History

- Veterinary Sciences Tomorrow

- The Veterinary Quarterly

- Ars Disputandi

- Studies in Mycology

- Ancient Narrative

- Neerlandistiek.nl

- Cahiers voor Geschiedenis en Informatica

Journals in co-publishing

- Pedagogiek

- Tijdschrift voor Hoger onderwijs

\section{Publication sites}

- Design Research Internet Magazine

- Interactive Publication: European Journal of Transport and Infrastructure Research

- Syntactic Microvariation 
Grey Literature

- Newsletter Historia \& Informatica

- Unitwin Series for Namibia

\section{Signal Hill}

Signal Hill is a recent initiative of the University Libraries of Utrecht and Delft (initiators of the Roquade project) and Firenze University Press to stimulate interaction, communication and co-operation between academic e-press initiatives (http://www.signal-hill.org).

Mission

Signal Hill is a European partnership for academic publishing. The aim of the partnership is to create a community of practice for organisations engaged in electronic academic publishing to enable them to combine forces and share their experiences.

Initiatives have been introduced in several countries in Europe to support academic publishing without involving traditional commercial publishers. Information technology is being used to create an infrastructure to facilitate and promote academic publishing by scientists and scientific communities, with an emphasis on communication. The main objective of some of the initiatives is to promote new models of academic publishing; others have as their core objective to make the scientific output of their own institution or of a well-defined group of scientists widely available. If this kind of publishing is not free, at least it is less expensive and user-friendlier than traditional forms of publishing. New business models are still being developed and elaborated.

Signal Hill targets university presses, libraries and research organisations that are involved in electronic academic publishing.

The objectives of the partnership are:

- to create awareness of innovations in academic publishing;

- to share experiences with new methods of (supporting) academic publishing;

- to share support software for academic publishing;

- to share access to publications;

- to present linking facilities;

- to set up a network of facilities for printing on demand.

Signal Hill will evolve into a Europe-wide community of practice for electronic academic publishing. Relationships will be established with comparable networks and organisations outside Europe, such as SPARC, and organisations such as LIBER have been asked to lend support.

\section{Activities}

The following activities are currently being pursued:

- a survey of European activities related to electronic academic publishing (starting from March 2001);

- the organisation of an intranet for participating organisations to facilitate the exchange of experiences (result: April 2001);

- the organisation of a European conference to promote the aims of the partnership (February 2002 in Utrecht);

- a project investigating new business models; 
- an active search for new partners.

\section{Members}

Membership of Signal Hill is open for organisations such as university presses, libraries and research organisations that are involved in electronic academic publishing. Their missions should have at least the following elements in common:

- they are part of (an organisation within) the academic community;

- they concentrate on electronic publishing, possibly offering the option of printing on demand;

- they are non-profit;

- their publications are offered at the lowest possible price, and preferably free of charge;

- the copyright remains with the authors.

Membership is free of charge. Members of Signal Hill are expected to provide information about their activities, their plans and their experiences. This information is only available to the other members of the partnership on the intranet.

\section{Conclusion}

The experiences with the Roquade project thus far show that there is a new and real challenge for university libraries, to be engaged in the support of electronic publishing. This challenge is real because projects like Roquade are not only initiated on the basis of a strategic analysis by the library management, but even more so because scientists within our university have come to the library asking for the support they need to make a successful transition to electronic publishing and new publishing models.

It is impossible to predict what the future for academic publishing will look like. Furthermore, it is rather shortsighted to declare a standard for what the future should look like. Therefore, one should not impose one preferred model onto scientific communication. The academic community should take initiatives that, in a flexible way, facilitate new structures in scientific information processes, which are favourable for the academic community itself. Roquade is such an initiative. The project will have benefits for the academic community, as well as for the libraries that take the initiative.

Its intended benefits for the academics (both authors and readers) can be summarised as follows:

- rapidity

- quality

- transparency of the reviewing and publishing process

- positive attitude of scientists towards digital publishing

- technological innovation

- fair price

- usage statistics

The intended benefits for the library are:

- Opportunities for applying gained experience of digital publishing and for exploiting the existing infrastructure in order to support the information and communication requirements of researchers.

- Better position in negotiations with publishers active in all fields in order to slow down the rising of subscription rates as well as improving financial benefits for the academic community in the long run.

- International co-operation. 


\section{References}

Savenije J.S.M. 1997 New competitors for the publishers: The cobbler and his last. Electronic Library, 15 (6), 431-435.

Savenije J.S.M. \& Grygierczyk N.J. 1999 The Roquade project: A gradual revolution in academic publishing. Proceedings of the 23rd International Online Information Meeting. Learned Information Europe Ltd, 211-216.

Savenije J.S.M. \& Grygierczyk N.J. 2000 The role and responsibility of the university library in publishing in a university. LIBER Quarterly 10 (3), 312-325. 



\title{
Legal Deposit of Electronic Publications
}

\author{
Giovanni Bergamin \\ Librarian \\ National Library of Florence, Italy \\ E-mail address: giovanni.bergamin@bncf.firenze.sbn.it
}

\begin{abstract}
The Guidelines of IFLA for long term preservation and access to electronic publications, published in 2000, stimulate for an appropriate legislation of legal deposit in the digital context. The National Library of Florence is involved in an initiative for the voluntary deposit of offline electronic publications (mainly CD-ROM) for the Project EDEN and of online electronic publications for the electronic resources (ER) of Firenze University Press. Trends and issues are described
\end{abstract}

Concerning the legal deposit of electronic publications the International Federation of Library Associations and Institutions (IFLA) has recently published (October 2000) a revised edition of the Guidelines ${ }^{1}$ of 1981 . This revision dedicates an entire chapter to electronic publications in order to help every country to provide an appropriate legislation for this kind of publications.

In that chapter, it is clear that the real challenge lies with online publications and, therefore, a precise methodological indication is given. It is important that the library in which the publication is deposited should have both the legal and technical support to archive and control the publications deposited there.

Offering access alone to the publication is not enough. In the infrastructure which assures the preservation of publications over a lengthy period of time. ${ }^{2}$

Electronic publications can be of various types and, in particular, resources of a dynamic type are objects which are very difficult to deposit. It is important, however, that legislation provide for all types of electronic publications (the technical solutions will be found).

In addition, legislation must include provisions which give full control over the deposited resources: the possibility of using all the techniques of appropriate preservation (migration, emulation, etc.) without infringing upon copyright laws.

Finally, legislation must provide "that a site licence be granted with the deposited electronic material, both off-line and on-line, in order to allow researchers to search the electronic sources for private and non-commercial use". On this point the Guidelines also formulate an innovative proposal for remote access, that is to say, that legislation should "include provision for at least one registered user at a time to access the material as long

\footnotetext{
${ }^{1}$ Guidelines for legal deposit legislation / by Jules Larivieère. - A revised, enlarged and update edition of the 1981 publication by Dr. Jean Lunn. - Paris: UNESCO, 2000. URL: http://www.ifla.org/VII/s1/gn1/ gnl/legaldep1.htm (Last accessed on November 11, 2000).

${ }^{2}$ Ibid.
} 
as he/she has demonstrated through a signed agreement that he/she is performing private and non-commercial research". In fact, restricting access to this kind of publications only from the premises of the deposit library is not a reasonable solution considering that the deposited publication could serve via network the whole plane. The proposal is in fact "a compromise based on the balance of rights between citizens and publishers".

While awaiting legislation which will take into account what has been proposed for the electronic publications at international level (but Italy is still awaiting a new legislation regulating also legal deposit of printed publications) the Biblioteca Nazione Centrale di Firenze (BNCF) has set up an initiative for voluntary deposit.

As well as the $\mathrm{EDEN}^{3}$ project (voluntary deposit of offline publications - mainly CDROMs) and, thanks to the recent agreement with the Università degli Studi di Firenze, the Biblioteca Nazionale Centrale di Firenze is concretely beginning the deposit of online publications. ${ }^{4}$

The Electronic Resources (ER) of the Università degli Studi di Firenze (monographs or serials) will be:

- catalogued in the Union Catalog of the network of the Servizio Bibliotecario Nazionale (SBN);

- inserted in the common workflow of treatment and management of the digital archived objects of the Biblioteca.

The BNCF will experimentally give - to a resource deposited - a National Bibliographic Number (NBN) which can be used as a Uniform Resource Name (URN). On the side of the identification of electronic publications it is important here to point out the work of the national bibliographies and libraries which have proposed ${ }^{5}$ the NBN or the use of the number of the national bibliography as a URN because:

- it has a good tradition;

- the resolution service (from URN to URL) is guaranteed by the national bibliographies which institutionally have the aim of documenting the editorial production of a country.

As we know, the electronic publication is a very fragile object, it can be easily counterfeited and poses problems of preservation over a lengthy period of time.

Following experiences diffused on an international level the authenticity of deposited publications - rather than being stamped with a rubber stamp and ink - will be assured by a MD5 digital signature (RFC $1321^{6}$ ). For example anyone can verify that the copy of

\footnotetext{
${ }^{3}$ The text of the agreement between the Ufficio Centrale per il Beni Librari and the associations of publishers can be found at URL http://www.bncf.sbn.it/Progetti/edi_ele/home.htm (Last accessed on November 11, 2000).

${ }^{4}$ For the text of this agreement see URL http://www.bncf.firenze.sbn.it/progetti/Europe/index.html (Last accessed on November 11, 2000).

${ }^{5}$ Hakala, Juha. Using National Bibliographic Numbers as Uniform Resource Names. - Internet draft which expires on 30 July 2001 at URL http://www.ietf.org/internet-drafts/draft-hakala-nbn-01.txt (Last accessed on November 11, 2000).

${ }^{6}$ URL http://www.ietf.org/rfc/rfc1321.txt (Last accessed on November 11, 2000). "A message digest is a compact digital signature for an arbitrarily long stream of binary data. An ideal message digest algorithm would never generate the same signature for two different sets of input, but achieving such theoretical perfection would require a message digest as long as the input file. Practical message digest algorithms compromise in favour of a digital signature of modest size created with an algorithm designed to make preparation of input text with a given signature computationally infeasible. Message digest algorithms have much in common with techniques used in encryption, but to a different end; verifications that data have not been altered since the signature was published". This is of course a minimal level of authentication: solutions like digital certificate and digital notary timestamp will be implemented as these solutions will become more widespread in terms of protocols, services, standards and applications.
} 
the publication in hand is not counterfeited by comparing the signature calculated by the Biblioteca (and published on the OPAC of the BNCF) and the signature that the user himself can calculate.

The most difficult part for the BNCF remains providing depositories for archiving these publications. Considering also the large amount of publications which will have to be archived and managed (even from the point of view of the changes in technology) it is appropriate that the depositories provide the maximum automated functions (consider for instance - the amount of the administrative and structural metadata that must be gathered). Not only, the design of this depository must be based on an independent model of the various technologies of archiving (storage) which, as is well known, change rapidly.

Adopting what had already been experimented in the Networked European Deposit Library (NEDLIB) project ${ }^{7}$, the BNCF has created a prototype for a Deposit System for Electronic Publication (DSEP). It is a system based on the Open Archival Information System (OAIS) model.

The OAIS model adopted as reference model by several important experiments in the world of libraries and archives, such as NEDLIB and CEDARS ${ }^{8}$ in Europe, and PANDO$\mathrm{RA}^{9}$ in Australia, attempt to provide this conceptual framework of reference: it is an ISO Draft International Standard (DIS) released in June 2000 and developed by the Consultative Committee for Space Data Systems (CCSDS): an ISO international group of space agencies like NASA, ESA, etc..

An archive OAIS compliant defines itself as "an organization of subjects and systems that has accepted the responsibility to preserve information and make it available for a designated community". As reference model, OAIS provides a strong basis for the development of consistent new standards and specifications in the context of digital archiving as well as the promotion of a major awareness, on the part of the vendors of technology, of the requirements for preserving electronic documents over a long time. ${ }^{10}$

The agreement with the Università degli Studi di Firenze has begun to show results. The BNCF is about to sign agreements both with other universities and private publishers.

In addition, thanks to this agreement, the BNCF is beginning the Project EUROPE which has as its objective the Italian implementation of the results of the NEDLIB project.

Some (possible) conclusions.

It is true that many online publications are complex and difficult to archive. This, however, cannot be a valid reason for not archiving even the less complex ones. In the long run, the problem of archiving is a problem that regards not only libraries (it regards everyone: from the archives of the public administration to our own personal archives). It would be wrong for libraries to create local solutions.

It is necessary, however, to begin working without waiting to have all the solutions.

\footnotetext{
${ }^{7}$ URL http://www.kb.nl/nedlib (Last accessed on November 11, 2000).

${ }^{8}$ URL http://www.leeds.ac.uk/cedars/ (Last accessed on November 11, 2000).

${ }^{9}$ URL http://pandora.nla.gov.au/index.html (Last accessed on November 11, 2000).

${ }^{10}$ Reference model for an Open Archival Information System, White book, Issue 5, April 21, 1999 at URL http://ssdoo.gsfc.nasa.gov/nost/isoas/ref_model.html (Last accessed on November 11, 2000).
} 



\title{
Emis \\ The Involvement of EMS in Publishing Mathematics
}

\author{
Bernd Wegner \\ Editor-in-chief \\ Zernblatt fur Matematik \\ Technical University Berlin, Germany \\ E-mail address: wegner@math.TU-Berlin.DE
}

\begin{abstract}
Literature databases, scientific journals and communication between researchers on the electronic level are rapidly developing tools in mathematics having high impact on the daily work of mathematicians. They improve the availability of information on all important achievements in mathematics, speed up the publication and communication procedures and lead to enhanced facilities for the preparation and presentation of research in mathematics. The European Mathematical Information Service (EMIS) tries to bundle several of these facilities in a joint offer, to extend them by innovative components and to distribute them in a system of replicators for the benefit of mathematicians worldwide. EMIS has been founded by the European Mathematical Society (EMS) and is run under their supervision with support from the office of Zentralblatt $M A T H$ in Berlin. It is based on the voluntary supply of input from sources distributed worldwide. The aim of the talk is to introduce to the different offers collected in EMIS like the databases $M A T H$ and MATHDI, the preprint index MPRESS, the project to install the EULER search engine for distributed sources, the JFM-project for the indexation and digitalization of classical literature in mathematics and most importantly the Electronic Library, offering a variety of freely accessible electronic journals in mathematics.
\end{abstract}

\section{Introduction}

The increasing development of electronic devices for the publication of papers and books in mathematics lead to a drastic change in the communication facilities between authors and editors, to new ways of distributing mathematical publications to research mathematicians - like electronic journals, and to an extension of the offers of information on mathematical research to the mathematical community. Confronted with a permanently increasing number of research papers and high production and distribution costs for mathematical journals and monographs, it becomes more and more difficult to get access to these publications. Electronic publishing provides better facilities for these purposes. It reduces the production costs for the preparation of the articles and improves the methods to find his way through the tremendous bulk of mathematical research papers. It enables reviewing journals to speed up and improve their procedures.

In the following we shall report on aspects related with methods of electronic publishing and electronic communication by exhibiting offers provided by the Electronic Library 
of Mathematics ElibM in EMIS (European Mathematical Information Service) as an example which may indicate a good model for the future. This refers to the production and distribution of electronic publications in mathematics, to electronic access to evaluated information on all mathematical papers as well as to general search facilities helping to find interesting documents in mathematics. Last but not least it should be pointed out that ElibM is the core for a growing offer of mathematical journals in a bundle without any subscription enforcement.

\section{The General Concept of ELibM}

The idea to develop the European Mathematical Information Service EMIS was born at the meeting of the executive committee of the EMS (European Mathematical Society) in Cortona/Italy, October 1994. In ElibM as far as possible peer-reviewed mathematical articles should be provided. The whole enterprise was only based on the enthusiasm of some mathematicians trying to build up an alternative to high-priced commercial publishing. They were lucky to find technical support for their project from the editorial staff of Zentralblatt MATH. The success of ElibM heavily depended and still depends on the generous approval from several editors to make their journals freely accessible in ElibM. They were lucky to find very quickly voluntary partners to care about the distribution of the content. No considerations about competing portals were made at all. Just the positive response from partners prepared to give content to ELibM showed that there was a gap they were able to fill.

Hence as a next step it was decided almost immediately to set up a system of electronic servers in Europe for Mathematics under the auspices of the EMS, and this was extended very soon to the current version of a central server collecting mathematical information and distributing this through a world-wide system of mirror servers. In June 1995 ELibM went on-line under the URL http://www.emis.de/. The first mirrors were established very soon. See [2], [3] and [4] for first reports on EMIS as the general environment for ELibM. To distribute EMIS through a world-wide system of mirrors, where the full content of the service will be available and updated periodically, improves the accessibility of EMIS, and it simultaneously is important for the safety of the data and their archiving. This is in contrast to several other offers which are eager to maintain the exclusive control over their offer. More than 40 mirror sites have been installed so far. There are two in Italy, the one in Turin belonging to the first in this group and the one in Lecce just being installed.

Commercial publishers also start to think about portals in mathematics, and some of them even want to sell their bundles of electronic journals as a kind of portals or comprehensive database in mathematics. But because of the commercial aspects this is not comparable with EMIS, and the concept of these activities is a different one.

\section{The Offers in ELibM}

There are four sections in ELibM: journals, proceedings volumes, monographs, and collected works. In order to guarantee that the electronic publications stored in ELibM meet the requirements satisfied by articles in print journals, the decision on the inclusion of journals, proceedings or monographs is taken in accordance with the Electronic Publishing Committee of the EMS. Hence no documents will enter the library which have not been evaluated and recommended by a referee within the editorial procedures of the 
corresponding journal or series. This is in particular important in order to rule out the current reservations of scientists who have the opinion that electronic publishing will damage the quality of research publications.

The section on Electronic Journals contains purely electronic journals as well as electronic versions of print journals (dual journals), both nearly at equal parts. The purely electronic journals are produced elsewhere and ELibM plays the important role of a bundling distributor. The installation of electronic versions of print journals depends on the technical facilities of the editors of these journals. Preferably, the offer of the electronic version is installed at the site of the editors, such that a mirror of the journal can be taken over by ELibM. Support for this is provided accordingly. Most of these print journals are published at a low-budget level, and hence the risk to loose subscribers to the print version due to the free electronic offer currently had been considered as low by them. Some of them give the electronic offer with a delay to EMIS such that the earlier availability will serve as an advantage of the print version.

But to be honest, there may be a change in the near future. The electronic information will be the more attractive service. The electronic installation will be the first item available and this has to be reflected in the access policy. Nevertheless, the offer in EMIS will remain as free as possible, and low budget models for such a change will be provided. One idea may be a charging of the access to the electronic offer for dual journals in an intermediate period.

Though there obviously is no chance to offer free access to electronic versions of the journals in mathematics published by commercial publishers, EMIS succeeded to get the agreement of a series of quite established journals to be made freely available in its library, as can be seen from the samples given in the subsequent list. The idea behind the selection is to show that contributions to EMIS come from many parts of the world. The purely electronic journals are marked by an (e):

Annales Academiae Scientiarum Fennicae Series A. Mathematica (Helsinki), Algebraic and Geometric Topology (e) (U.K.),

Beitraege zur Algebra und Geometrie / Contributions to Algebra and Geometry(Berlin), Commentationes Mathematicae Universitatis Carolinae (Prague),

DOCUMENTA MATHEMATICA (Bielefeld),

The Electronic Journal of Combinatorics (e) (U.S.A.),

Electronic Research Announcements of the AMS (e) (U.S.A.),

Geometry and Topology (e) (U.K.),

Homology, Homotopy and Applications (Tbilisi),

Journal de Théorie des Nombres de Bordeaux (Bordeaux),

Matematicki Vesnik (Belgrade),

Mathematical Physics Electronic Journal (e) (U.S.A.),

Revista Colombiana de Matematicas (Bogota),

Seminaire et Congres (e) (Paris).

More or less all freely available purely electronic journals in mathematics are mirrored in EMIS. The current total number of journals in ElibM is 45 and 9 are about to be installed soon, some of them coming from Italy.

The access to the journals is organised quite conventionally. On the home page of EMIS a list of mirrors is provided where the site with the presumably most convenient access can be clicked. Then a choice can be made, to enter the ELibM through the short list of journals without graphics or to use the full display of these items. The first one is preferable, if the choice of journal is clear already and if one wants to avoid the lengthy transfer of the graphical data associated with this journal. The full display also contains background information on the editorial policy of the corresponding journal and instruc- 
tions how to submit an article. In cases style files for such a submission can be found on this level.

Having made the choice of journal one wants to read, the level of the contents will be reached. This is organised as usual. With the table of contents information on the offer of files is given. In all cases DVI- and Postscript-files will be available, sometimes also TEXfiles can be found in addition to that, and in cases geometric material is stored in separate files. PDF is coming up rapidly and will be an obligatory offer in the near future. Clicking one of these files, its content is transferred to the computer of the user and can be viewed there. Also printing or storage of these files will be possible at the site of the user, but he is requested to respect the copyright according to the rules of the corresponding journal. The access to the section of Proceedings Volumes is organised in a similar way.

At present we have only a few Proceedings Volumes in EMIS, though electronic publishing will be the choice for the future because of their low selling rates on paper. If contributors to proceedings really are interested to make their work public in a reasonable way, they will see that electronic publishing will be the only way. ELibM is just the appropriate system for a good distribution of such publications in mathematics. This is underlined by the present access numbers which are growing continuously.

As samples for Proceedings those from both meetings on Quaternionic Structures in Mathematics and Physics (Rome, 1999 and 1997) and from the International Mathematical Congress of Mathematicians (Berlin, 1998) should be mentioned. As a monograph with not-printable electronic enhancements "Cusps of the Gauss map" by T. Banchoff et al. is remarkable. But also S.S. Kutateladze "Russian - English writing. Advice to the occasional translator" can be found in ElibM. A big historical achievement are the digital editions of the collected works of Hamilton and Riemann by David R. Wilkins.

\section{Projected Improvements for ELibM}

Admittedly, after five years of EMIS some of the above features will have to be modified. The request for PDF-files will become a must, because the preinstalled readers at electronic access facilities in libraries and desktop-computers of research mathematician already point into that direction. For the current offer in many cases special measures have to be taken for making the article readable or printable. Decisions about affordable systems for assigning digital object identifiers (beyond DOI) have to be taken to enable a richer linking system between electronic articles. Probably the activities for installing a distribution system of URNs will be a good choice. Having such a lot of articles in the library, better direct access to the articles will have to be organised, improving the initial browsing of journals and their contents. One choice may be the EULER service (see [5]).

As mentioned earlier, the upcoming method to offer the electronic version of an article as soon as the article will be available in final form ("online first") will have impact on the free access to the installation of the dual journals in ELibM, i.e. journals which provide both a printed version and an electronic version. They will not be able to survive from what they get for the paper version, when online will be declared as "first class" and print will be still important, but second class only.

But anyway, EMIS and in particular ELibM will remain non-profit during such a transition, and provide as much as possible on the free level. ElibM will try to co-operate with similar offers, like they will be available through project Euclid and the upcoming activities of low-budget publishers supported by universities and libraries. Very soon some 
integrated access to the holdings of ElibM and those of digitisation projects like ERAM (see [1],[6]) will be available. Finally, in addition to the current archiving through the mirrors more precise arrangements for the long-term preservation of the contents of ELibM will have to be established. Here co-operation with some comprehensive mathematics libraries like SUB Goettingen is on the schedule.

\section{References}

[1] Becker H. \& Wegner B. 2000 ERAM - Digitisation of Classical Mathematical Publications, Proc. ECDL 2000, Lecture Notes in Computer Science 1923, 424-427.

[2] Wegner B. (1997a) EMIS and its Electronic Library. Vichysl. Tekhnol. (Comp. Technol.) 2 (3), 25-30.

[3] Wegner B. (1997b) EMIS i ego elektronnija biblioteka. (Russisch) Nauka b Sibiri 21, 4.

[4] Wegner B. 1998 Electronic Publishing and Electronic Publications in Mathematics. Progress in Mathematics 169, 315-337 - RT at EMC 2, Budapest.

[5] Wegner B., EULER -a DC-based Integrated Access to Library Catalogues and Other Mathematics Information in the Web. Seventh International Conference "Crimea 2000" Libraries and Associations in the Transient World: New Technologies and New Forms of Co-operation. Conference Proceedings. Sudak, Autonomous Republic of Crimea, Ukraine, June 3-11, 2000, Volume 1: 264-267.

[6] Wegner B. ERAM - Digitalisation of Classical Mathematical Publications. Seventh International Conference "Crimea 2000" Libraries and Associations in the Transient World: New Technologies and New Forms of Co-operation. Conference Proceedings. Sudak, Autonomous Republic of Crimea, Ukraine, June 3-11, 2000, Volume 1: 268-272. 



\title{
The Role of The Publisher in Scholarly Communication
}

\author{
Anthony Watkinson \\ Publisher consultant \\ Visiting Professor in Information Science \\ City University London, UK \\ E-mail address : Anthony.watkinson@btinnet.com
}

\begin{abstract}
The Internet has begun a transformation of scholarly communication that is ongoing. This does not mean that the roles of intermediaries in the information chain between author and reader no longer add value. The publishing role which essentially involves organisation of selection and certification is just as important. In many ways both these functions are even more important in the electronic as in the print environment because it is so much easier to put rubbish on the web. There is also the problem, which may or may not be a temporary one, of the costs of moving from all print to all electronic through a hybrid situation in which print and electronic co-exist and costs are higher rather than lower. There is also as yet no serious sign of a movement from payment by user to payment by author - however reasonable the shift in cost may be in theory. Cost recovery alone is not enough but there has to be a suitable level of surplus for investment in changing technology and to make the publishing process self-sustainable. This is as true of book publishing as of serials publishing. Dislike of commercial publishers should not blind librarians and others to the basic economic facts of life that impact on new publishing models as well as old ones.
\end{abstract}

\section{Providing the context}

This is the published version of a presentation delivered in Florence in March 2001.

In order to maintain something of the informality of a presentation I have avoided detailed references that would footnote assertions but I am happy to back up anything stated in what follows for those who are interested. I have provided in the last section of this chapter a bibliographical note, which I hope will be useful.

The aim of the presentation was to argue for the continuing existence of publishers and to explain what publishers really do but in the context of the ambition of the conference organisers.

My interpretation of this ambition is that it is to show how, in the digital environment, university presses can re-interpret the publishing function in a way that applies the new possibilities to the old problems of one scholar reaching another scholar with his or her message. It is often said that one result of the move from print-based to digital publishing is that practices, refined over decades and sometimes centuries, are no longer valid. Publishing characteristically works by precedent and, if the presumed discontinuity is 
valid, painful rethinking is necessary. My own position is that change management must be central to the management of traditional organisations, however painful, but that the failures of dot.com companies seem to demonstrate that you cannot afford to ignore experience.

My concern is not with all aspects of academic activity. The research function is different from the teaching function and I am only here concerned with the former. Academic research is communicated by either monographs (primarily in the humanities and the social sciences) or journal articles (particularly in science). This type of communication, which I like to call R2R -researcher to researcher- should in my view be a special concern of a university press, though as well shall see in section four, this is not the current position in the print environment.

However I want to emphasise that, throughout this chapter, I am assuming that scholarly communication is becoming and soon will become intrinsically electronic. Print will remain but it will almost always be a subset - a matter of "printing out". Print will remain because it is usually most convenient to read, though not to search, in print and not on screen.

\section{Should publishers exist?}

In print there is an information chain between author and reader and both libraries and publishers are intermediaries in that chain. The Internet, as is often pointed out, tends to replace a linear relationship with a more complex set of connections or by direct contact resulting in disintermediation. In the 1990s disintermediation was argued by many to be the inevitable result of general Internet usage. Not only was the role of the publisher in consumer publishing seen as no longer necessary when author could contact reader directly, where there is still an active if not generally influential self-publishing movement, but the same style of thinking was evident in discussions of scholarly communication.

I am writing in the past tense but, even though at least my spell checker does not recognise the word, ideas of disintermediation are still actively propounded in some circles. Another speaker at this conference will explain how the Open Archives movement renders one sort of primary publishing irrelevant. The chief executive of one large medical publishing company in the UK holds the same views. Some speakers associated with SPARC and similar library sponsored bodies such as JISC in the UK have asserted that one of their aims is to bring author and reader directly together. There have also been plans to "seize control" on behalf of the "Academy" various components of the traditional publishing role such as the proposal (not yet realised) to place the reviewing/certification function either with the (US) universities or with (US) learned societies.

My argument is that the intermediaries continue to add value, and indeed may add more value in the digital environment. I argue that the Internet has had a significant impact on informal communication but the impact of web-use on formal communication has been different. Essentially access is promoted but roles have not changed. I shall return to the claim that publishers may be providing more value now than they did in the fourth section. I will attempt to unpick some of those other assertions concerned with essential continuity in the next section of this chapter.

Intermediaries, though I am tending to use this term in the academic context to refer exclusively to publishers and librarians, also includes other players like book vendors and subscription agents from the old dispensation as well as new companies in both book and journal sectors, companies like ingenta and NetLibrary to name two obvious enterprises. 
The key to the value added here is aggregation and I shall return to the importance of aggregating again.

I also want to make two points about what I regard as misleading and distorting assumptions underlying attacks on the publishing role.

I have already glanced at the concept of the Academy. The way the term is customarily used, for example by the Association of Research Libraries (ARL), assumes that libraries are part of the Academy and/or represent the Academy, whereas publishers do not. My contention is that publishers and librarians both represent the academic community, the former of the academic as author and the latter of the academic as reader or user. As both intermediaries know academics tend to have a split personality..

The second point is also contentious. It is common in library circles to make a distinction between "commercial publishers", who are bad, and non-profit (US) or not-for-profit (UK) publishers, who are good. For reasons, which will become clear below, I prefer the UK usage. All publishers, who want to survive as publishers, have to make a profit or a surplus. SPARC, through the BioOne initiative, have had to bail out the smaller US learned societies who did not aim for or achieve a surplus. It is obvious that some (but not all) of the profits made by commercial publishers go to shareholders. It should not be forgotten that quite a bit goes in tax too. For many librarians the word "shareholder value" has become a dirty word, but is return for investment (see the next section) unfair or unreasonable? The trading surplus (not taxed) of not-for-profits goes back into the academic community directly, either to universities (though few university presses make money) or to underpin the activities, such as conferences, of learned societies or underwrite their membership fees.

Nevertheless the policies and preoccupations of all publishers are much the same. They belong to the same organisations and work together over initiatives such as CrossRef. It is not always noted that $60 \%$ of the publishers participating in CrossRef are not commercial companies. Commercial publishers came into being because university presses and learned societies were failing their author communities and were not responding to the increase in the numbers of publications following the huge increases in research funding of the second half of last century. There were other reasons why influential academics turned to commercial publishers. One example is that they recognised the globalisation of scholarly discourse. This was and is less true of social sciences and humanities but in science leaders of scholarship are leaders across the world. Nationally based learned bodies (the norm) could not (and still cannot always) turn to the best editor or author. Commercial publishers were/are successful because they were/are doing a good job and there is a lot of learn from the way they operate. Many not-for-profit publishers have. However they have no inalienable right to exist: the authors will vote with their papers.

\section{What do publishers do?}

This section is selective. The principle for selection refers back to my statements concerning relevance in the first section. In particular I am looking to the electronic future rather than the print only past. There will be more about journals because there is more to say about journals than scholarly monographs. Monographs are only just going online in any numbers.

It also seems to me that what publishers do cannot be separated from the costs of what publishers do. After all it is the costs as expressed in the rate or price downstream which is central to the debate. It is customary in contributions of this sort to downplay 
the role of the publisher in production and marketing/distribution. The argument is that the Internet has minimised this function and that publishers have come to realise what their core competencies are, i.e. the certification function.

Let us first look at the core competence from the user perspective. Karen Hunter of Elsevier Science (the largest scholarly publisher) has written a succinct description of how scientists use academic journals:

"Academic science researchers publish to establish their claim at a specific time to a specific result. They publish to gain other forms of recognition (such as promotion and tenure) that require publication. They publish in order to have independent certification of the results and to have those certified (refereed) results archived in perpetuity. Finally they publish to communicate with those who may be interested in their works today - not the circle of cognoscenti but researchers in related fields, researchers in less well-connected institutions and students working their way into the inner ring."

Social scientists and those working in the humanities and arts, who on the whole look to writing books rather than articles, would emphasise the communication function even more but they would agree on certification.

It is generally accepted that organising peer-review and its context is the central and, some would say, residual function of the publisher or some entity that exercises that role. That is not my experience. Publishers organise the whole process and it has not become easier. There are so many technical questions to solve from the provision of author templates to the provision of an online offering which will satisfy customers and end-users. It would be great for publishers if authors did all the work in the digital environment but it is just not the case in most disciplines and circumstances

Most of the money-cost and the costs in staff-time of the publishing process are for pre-print operations that precede printing, binding and distribution. These do not disappear, though there can be some savings in some cases. Peter Boyce (see section five) has explained how these savings can be made in one very special situation, the astrophysical community. However his publisher, Chicago University Press, makes clear how complex a problem they faced and still faced in getting to this stage with this particular journal (The Astrophysical Journal).

In parenthesis it is worth stating that, although referees of journal articles are rarely paid, most editors of journals are rewarded sometimes handsomely while costs of financing their back-up and office costs increase significantly. A major publisher reckons such editorial costs as 10 to $15 \%$ of total costs associated with a journal.

Print plus electronic is bound to be more expensive than print publishing only for both publishers - and for librarians. For most publishers it is (alas) necessary to offer both formats.

Not only are publishers making available content in print and electronic but the sheer cost of going online cannot be overestimated. Below is an edited private communication from a director of a major journal publisher in answer to a suggestion that journal publishing was risk free and easy:

"Are they serious? 40\% of the jobs in our company did not exist 4 years ago. That requires significant investment in the training and development ofstaff and management of change). The investment required in 'going electronic' and delivering the functionality which customers EXPECT has and continues to be very significant (e.g. Z39.50 compliance will be an extra investment this year which will not directly 
bring in new sales). Early investment in electronic content was susceptible to being of little or no benefit depending on how the market developed - there were no standards to follow - consider the millions of research funds (private and public sources) which went into early projects which led to no appreciable return. It is anything but a doddle. Worldwide adoption of technology to access electronic content is very uneven requiring duplicate channels, efforts and investment. The new technologies have created opportunities - for the user, customer, intermediary, author, publisher, etc - but the implications are still being worked through."

This is only some of a long list of costs involved in transformation. The bottom line is investment and more investment. Publishers invest.

Journal publishers have been tangling with these questions for some years with some put not total success. Scholarly book publishers have only just begun to work through the consequences of going online. A recent survey asked a group of publishers if they were up to speed in the digital environment. The following reply was both typical and significant:

"I feel we are in the forefront in this market - we are very active in knowing the issues and the people. But resources are difficult to find so we have not taken the plunge yet"

This quotation picks out both the lack of practical experience and the lack of investment (because scholarly monographs do not make enough money to allow for ploughing back of income) which presents special problems for any organisation hoping to facilitate communication at book length.

Most of this section so far has been concerned with the mechanics of publishing. The editorial role of publishers, as distinct from the role of the "certifying" academics they organise, is rarely discussed.

What is this editorial role? To my mind it has two main components. The first is selection and the second is content development.

Most publishers decide what is published. It is most obvious in the publication of books. 0Selection may be the responsibility of a board of distinguished academics or a group of series editors but in the end the publishing staff have to be certain that what they publish has a market (will sell enough copies) as well as a stamp of quality.

Mark Bide, one of the most detached commentators on publishing, wrote in 1999:

" The focus of every business model is the customer, and the one thing we are learning about customers for electronic information is that their behaviour is extremely complex and influenced by many different factors."

Part of the role of the publisher is not only to assess the market and advise on its needs but also to discern the changes of user acceptability in a changed marketplace.

In the case of journals the decision the content of the regular issues is in the hands of the editorial structure even if the publisher appoints the editorial structure. Where the selection is most obvious is in the decisions made to start the journal, change the frequency and extent and (sometimes) to stop, merge or sell the journal.

Mark Bide, again, made the following point in a Vista presentation:

"More publishers consider that content development is the most important thing that they will do in 2002 than did in 1997" 
This is not surprising. In the print environment publishers have always advised their authors on how to put a book together, what is relevant (according to their advisors) and irrelevant, and what it appears the marketplace is looking for. Mentoring in journals is the job of the editors and their referees. In the electronic environment content development is much more important because authors face the challenge and the opportunity of enhancing their message through the use of all sorts of features that are electronic-only, which especially includes links. The projects founded by the Mellon Foundation, which are referenced in section five, are facing up to the new modes of content development. The big initiative impacting on the way in which serials are used is the development of the Digital Object Identifier (DOI) and its main implementation CrossRef.

\section{What might publishers do?}

Firenze University Press convened the international conference, at which this presentation was given. The aim of the Press is to make use of the opportunities presented by the electronic environment to make scholarly communication more responsive and more effective. It is a "Digital" press. However it is not experimental in the narrow sense. Those developing this initiative are looking for a sustainable economic model.

The theme of this section is that librarians adopting the publishing role can bring special insights with them but they are foolish if they ignore publisher experience.

The history of university presses is not an encouraging one. There are of course huge exceptions (which need not concern us here) to a norm, which is an institution underfunded and with real problems serving their author community adequately. Although the information revolution has opened the door to new opportunities, most presses have not been in a position to seize them. Very few university presses publish journals, which is a severe disadvantage as the "monograph crisis" bites. Andre Schiffrin, an acerbic commentator on the US scene, wrote in 1999:

"As Peter Givler, executive director of the Association of American University Presses, elegantly phrased it to me, many universities can be said to be offering "negative" support." In other words, they have decided that their presses should be treated as profit centers, and have asked them to pay back to the university a percentage of their sales... The question is whether universities will choose to use their resources to help presses develop books that can serve their constituencies in intellectual exciting ways - or whether universities will continue to reduce financial support to presses, and, even, in some cases, demand payments from them."

What this means in practice is that many presses minimise their involvement in scholarly communication in favour of communication of scholarship outside academe. Universities tend to tell their presses to publish fewer monographs and more books of consumer interest and, in particular, local interest. There is nothing wrong with the latter aim but is it an appropriate mission for a university press?

Where Firenze and the other presses described by Bas Savenije of Utrecht, in another contribution to this book differ from this approach, is that they are wholeheatedly dedicated to helping authors/creators reach readers/users. Because in the scholarly arena authors and readers are essentially the same community, they (the new publishers) bring insights to their task from their experience as librarians. They hope to integrate, to concertina some of the processes and thus to serve scholarship better and at less cost. I think 
this is a fair statement of the aims of Firenze specifically and the anticipated Signal Hill community in general.

Since the growth in the usage of the web became of evident utility to the publishing process in the mid-1990s electronic gurus have argued for the collapse of traditional publishing structures in favour of new digital organisations. The big companies and learned societies, which were dominant then, are dominant now. They have adjusted and the newer (and smaller) companies have tended to go to the wall or be bought out. There are exceptions such as HighWire Press but they are not a publisher - rather a facilitator of publishing.

The economics of publishing remain the same whoever the publisher is. My argument is that for a new type of publisher to be successful, both this fact and what follows from it has to be taken into account and also lessons learnt from the successful companies, whether commercial or not-for-profit, have to be studied and acted upon.

My advice for the new university presses is as follows.

Get the economics right. SPARC tends to talk about set-up costs only. This assumes a stable situation as far as technology is concerned. In the digital environment, as we have explained above, the technological opportunities of authors and the technological demands of users, sometimes, though not always through their libraries are in a state of constant change and financing the change requires investment which has to be allowed for. If a new press is to be successful it cannot afford to wait and see. It needs to be quicker off the mark than the traditional players.

In a university situation many costs are hidden in overheads but there is no guarantee that some future bureaucracy will not insist on a "fair" allocation of overhead costs. Indeed the more successful a new press is the more likely that it is to be noticed, not just because it uses more of the resources concerned. Even if you are working with subsidies you still have to budget.

Just because a potential academic author works in the university, loyalty will usually only become a factor if rewards (royalties for book authors and remuneration for journal editors) are at least recognised and may be allowed for. Some of the new ventures are looking to sharing profits or even offering shares? I would venture to suggest that nonpublishers undertaking a publisher role ought to work to understand the psychology and preoccupations of the academic as author even if they understand the academic as user. It is important to discover what authors actually want in practice as well as in theory and whether it is cost-effective to provide what they say they wish. Journal publishers who went online early really believed that authors wanted to make use of all the opportunities presented by the digital environment, e.g. video clips. As yet take-up, when offered, has been minute. This was one of the big lessons of the SuperJournal project.

I mentioned the editorial functions of publishers in the previous section because it has been my experience that librarians do not understand these functions. A new press must achieve a reputation for high quality and international relevance if it hopes to sell its publications, whether books or journals, worldwide. Editorial intervention is needed. In particular I would argue that it is doing a disservice to scholarship and to libraries in particular if any journal proposed by senior local academics is started by the university press. Failure to ascertain the market has been a feature of many e-journal programmes e.g. those of JISC in the UK. It is often forgotten that publishers turn down many more proposals than they take on board and develop.

Some have argued that a publisher needs only to help scholars get their work on the web and that nature will take its course as search engines do their robotic work. I do not accept this view. I suggest that the information overload and the lack of obvious authority implicit in the Internet environment actually increases the need for the certification/vali- 
dation and navigation functions provided by publishers and librarians. This lack of confidence in the Internet is to my mind the main reason (not doubts about archiving and preservation) why e-only journals are not getting off the ground (usually) and why e-only monographs are likely to be even slower becoming a success. An American academic (J.W.Brodman) wrote perceptively last year:

"Our enthusiasm should, however, be restrained, as we attempt to solve a number of real problems associated with the electronic publication of academic materials. The first of these is legitimacy... The simple truth is that university faculty who sit on hiring, promotion and tenure committees do not yet consider digital text the equal of printed text."

Publishers have duties to authors that are only just coming to be recognised in the new circumstances and must be recognised if new relationships (as proposed) are not to work out badly. They will need to consider the protection of the moral rights of integrity and paternity much more actively in the future because the dangers to preserving authenticity are so much greater than they were.

Finally in the digital environment your publication has to be found and has to be delivered to through an interface that is acceptable to customer and user. You cannot leave discovery to chance. You cannot afford (literally) to be one of those small accounts which neither librarians not vendors want to touch. If you are a very big publisher you are an aggregator yourself but smaller organisations need to find ways of aggregating. One practical point relates to rights. The acquisition of copyright is not in itself important but publishers do need to acquire those rights from authors and others that they need to make publications available through as many channels as possible. Aggregating, though well developed in the journal arena, is in its infancy in book publishing. Some particularly interesting models are being developed in the USA under the auspices of the Edward Mellon Foundation. The most elaborate is to be found at www.historyebook.org.

Signal Hill (see another chapter in this book) has already recognised the importance for smaller publishers with common aims of working together to reach their audience. I would suggest that partnership with other types of intermediary is worth thinking of seriously. The commonsense of partnership is one of the nostrums of Internet thinking.

\section{Bibliographical note}

The most recent book on most aspects of scholarly communication, but with particular emphasis on the humanities and the social sciences is Communicating Research by A.J. Meadows published by Academic Press in 1998 (ISBN 0-12-487415-0). I have written on developments in global academic publishing in Publishing 2001: Attitudes to technological changes published by Bookseller Publications in 2001 (ISBN 0-85021-288-X). Some of the alternatives to and a description of the ways in which primary communication currently happen in science and medicine is set out in: The STM Information System: an analysis, Learned Publishing (1999) Volume 12 No. 1, 11-24 (Learned Publishing is now online, free of charge, at www.alpsp.org.uk/journal). There are few studies on electronic publishing of monographs. The most detailed treatment is in The Publishing of Electronic Scholarly Monographs and Textbooks by C. J. Armstrong and Ray Lonsdale (April 1998). This is available at www.ukoln.ac.uk/dlis/models/studies/elec-pub/elec-pub.htm but it is now a little out of date. My own study, which concentrates on monographs in the humanities, will be available this year online only. It is entitled Electronic Solutions to the 
Problems of Monograph Publishing. This study was conducted with the aid of a grant from the British National Bibliography Research Fund and information about how to find it will appear at www.lic.gov.uk/research/bnbrf/. The thinking of Peter Boyce (consultant to the AAS) is to be found at www.aas.org/ pboyce. By far the best description of journals publishing in science is found in Tenopir, Carol and Donald W. King: Towards Electronic Journals: Realities for Scientists, Librarians and Publishers (2000) available from the Special Libraries Association (ISBN 0-87111-507-7). 



\title{
Change in Univeristies: The Effects for Librairies, Academics and Publishers
}

\author{
Ian Winkworth \\ Director of Learning Resources \\ Library \\ University of Northumbria at Newcastle, UK \\ E-mail address: ian.winkworth@unn.ac.uk
}

\begin{abstract}
Publishing technology, worldwide communication, educational evolution, social change and Government policy, all provide drivers for rapid change in universities. The paper will explore how these changes affect the role in the scholarly communication process of academics, commercial publishers, university presses and librarians, and looking at the short-term and long-term effects of a greater (but far from exclusive) reliance on electronic media. In conclusion the pitfalls and requirements for the actors in this evolving scenario will be analysed.
\end{abstract}

\section{Drivers for change}

United Kingdom universities are changing quite rapidly. The main drivers for change are these:

Do it cheaper

Changing markets

More value for money

The 'customer' culture

Explicit Government expectations

Accountability

The Internet

For the last twenty years there has been a steady reduction in the funding received by universities for each student taught. At the same time the number of students has increased rapidly, from $10 \%$ of 18 -year-olds to $30 \%$. Markets have changed, as student preferences for different subjects have altered: for example fewer students wish to study physics and chemistry, and many more wish to study computing and business. University funding depends directly on being able to recruit students to the courses offered, so the balance of courses offered must change. In addition, UK universities have looked to set up activities in other countries. For example, Northumbria University has activities in many countries, including Russia, Thailand, Malaysia, China, and most recently in Italy, through a course in librarianship offered jointly with the University of Parma.

There is continuing pressure for more value for money from all public services -in current Government language "Best Value". For example, university core funding for 
research is allocated via a competition every four years -the "Research Assessment Exercise"- in which each academic has to suggest four "best" publications, on which the overall quality of their research will be assessed for quality and importance by a committee of subject experts. The ratings for all the academics in each subject area in each institution are assembled, together with other indicators such as the value of specific research grants achieved, having a good group research strategy, and the number of research students, and background research funding for the next four years is allocated accordingly for each subject area to each institution. This process illustrates two further features of the situation: the use of explicit performance measures to govern funding and thus to ensure accountability and responsiveness to explicit Government objectives; and the seeking of evidence of customer satisfaction - in this case from the relevant subject peer group. The same features exist in the quite heavy processes for checking on the quality of courses and teaching: student satisfaction is a key indicator. Good results for the quality of courses are essential: poor results will lead to loss of funding for those courses and to withdrawal of authority to offer the courses.

Finally, of course, there are the effects of the Internet, on which I will say more later.

\section{Effects for Scholarly Communication}

The combined effect of all these change-drivers on higher education is greater pressure on all staff. The number of staff of all kinds has steadily reduced, while at the same time expectations have risen. This means that there is a very critical approach to use of time. There is also now a substantial and continuing investment in IT, estimated at perhaps $10 \%$ of annual income at my own university, if software, hardware and IT staff costs are all included. This cannot be escaped, but must deliver benefits. This need for investment, alongside the funding reductions, means that "old" revenue costs -such as library costsare closely scrutinised. And the delivery of outputs, outcomes and customer satisfaction become more important here, too, together with ensuring that services benefit the new markets as well as the established clientele. All this points to rethinking of old roles and processes in respect of scholarly communication. In the light of these considerations, let us remind ourselves of the traditional scholarly communication process.

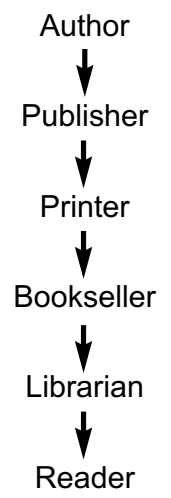

Fig. 1. The Traditional Scholarly Communication Process 
The paper will look at each of the roles one by one and what the future holds.

A really radical view of the future is shown in Fig. 2.

This kind of radical view was quite popular ten or fifteen years ago, but has proved totally unrealistic so far. With a little reflection, the reasons are not hard to see. Publishers, printers, booksellers and librarians collectively do more than act as passive conduits - they provide an intellectual, commercial and delivery structure beyond the technology. Also, print on paper remains a better medium for many purposes than any screen or any e-book yet invented. And local computer printing is expensive and cumbersome for large items and long runs. So, even the technological assumptions of the radical view remain unproven.

Fig. 3 is designed to emphasise the small part of the scholarly communication process, which is carried out by others than academics, and the even smaller part played by the commercial sector of publishing and bookselling. For the academic contributors of most of the input, commercial considerations are not high on the list of priorities, at least in respect of scholarly communication.

\section{Effects on the actors in scholarly communication}

I would now like to look at the effects of change in universities on each group of actors in the scholarly communication process. For the academics, the situation can be summed up as resulting in: less time; more demands; more change; more management pressure for specific outputs; more dependence on IT infrastructures and on collaborative and institutional effort; and tempting opportunities to diversify into new roles created or

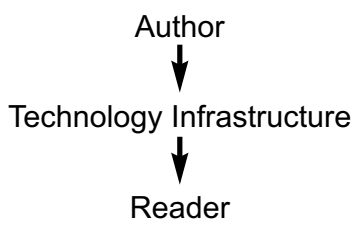

Fig. 2. A radical electronic view

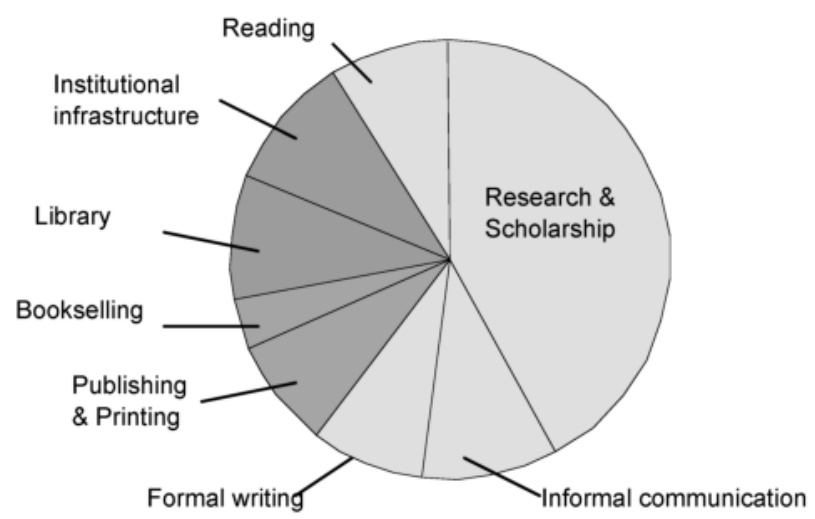

Fig. 3. Notional Shares of Costs of Scholarly Communication 
changed by the technology. This is a recipe for potentially serious stress and overload, if not carefully and sympathetically managed by institutions and individuals. The situation differentiates the workaholic innovators from the great mass of their colleagues, pulled along into the new world with some reluctance except where new innovations offer real personal advantages.

For publishers, including University Presses, their editing skills and risk capital are still required. Many University Presses operate at the margin where normal capitalism fails! I would emphasise the importance of the risk capital element of the publisher's contribution rather than the professional skills. Public sector bodies (like universities) are not good at understanding the need for this, nor how the process works. But for publishers, the new technology still brings many challenges - including the challenge of offering institutions and academics good value new products, which take advantage of the investment which universities have put into IT infrastructures. There is, however, ever more to publish, as academics achieve productivity gains from new technology. But the traditional economic models and income streams become destabilised: it is clear that the traditional printed journal is less and less viable - perhaps not a bad thing given the scattergun approach involved: subscribers buy vast amounts of paper, most of which remains safely unread in each of the copies sold. Surely, new technology will eventually offer us a better deal? But it is likely to be a deal based on "site licences" rather than copy purchases. And the new technology already offers 'bundling' possibilities - where a publisher can offer their full range of titles to a wider group at no greater cost to anyone than a subset of printed copies.

In this process it is the "middle" players -printers and agents- who are most likely to be cut out. The commercial printers are probably hardest hit, because they are closest to the core of the old technology which is being replaced. For them there will undoubtedly be less work (despite lots of books about IT), as their function is partly replaced by IT manufacturers.

For booksellers and agents there is more scope for diversification and for themselves applying the new technology to retain their business volume. In the city I work in, Newcastle, it is without doubt the case that the provision of bookshops is better than it ever has been. But the subscription agents will clearly lose that area of work which relates to the physical distribution of individual journal parts, as journals are distributed electronically instead. But there are new opportunities in managing the sale of electronic licences. For example, the UK higher education sector now employs a number of commercial and quasi-commercial agencies to manage delivery of bundles of electronic product to the sector, an activity that used not to exist. There will surely also be opportunities for managing the delivery of "e-books" to institutions and individuals.

The fundamental role of librarians as institutional managers of access to information sources remains unchanged. But the practical work involved is already shifting from a main emphasis on building up local, physical collections to a main emphasis on a variety of access licenses and agreements. Network and mail customers have already replaced some physical visitors, and this trend will continue. A corollary of this will be less emphasis on the library as a building and more emphasis on IT-based services. There is an additional new role of integrating print and electronic sources.

\section{Libraries}

To give a perspective on why all this is the case, I would like to look at some data on current library outputs, activities and expenditure. Fig. 4 lists some outputs of the library in a typical large UK university such as Northumbria. 
Personal visits

Book Issues

In-library book consultations

User electronic searches

Enquiries answered

Students instructed
$1.89 \mathrm{~m}$ p.a.

870,000 p.a.

$1.75 \mathrm{~m}$ p.a. (?)

300,000 p.a. (?)

240,000 p.a.

12,500 p.a.

Fig. 4. Typical Library Outputs in 1999/00

$\begin{array}{ll} & £ \\ \text { Staff } & 1.87 \mathrm{~m} \\ \text { Information Sources } & 1.73(£ 190,000 \text { electronic) } \\ \text { Equipment } & 0.37 \\ \text { Other } & 0.23 \\ \text { Revenue } & 4.20 \\ \text { Building maintenance } & 0.80 \\ \text { Building depreciation } & 0.55 \\ \text { Total } & £ 5.55\end{array}$

Fig. 5. Northumbria University Library Expenditure 1998-99

\begin{tabular}{|c|c|c|}
\hline Direct & Document Access & $9 \%$ \\
\hline Services & Information Services & $7 \%$ \\
\hline$(32 \%)$ & Study Facilities & $16 \%$ \\
\hline $\begin{array}{l}\text { Investment } \\
(51 \%)\end{array}$ & $\begin{array}{l}\text { Bookstock Provision } \\
\text { (including Buildings) }\end{array}$ & $51 \%$ \\
\hline Overheads & Management & $10 \%$ \\
\hline$(17 \%)$ & Other & $7 \%$ \\
\hline
\end{tabular}

Fig. 6. Library Activities: Shares of Total Library Costs

Note that the outputs are still predominantly print-related.

To understand a business, we need to relate its outputs to costs. Fig. 5 shows library costs as usually presented. 
Note that spending on "scholarly communications" as perceived by the publishing industry is only about one third of the total costs. But if we reapportion the costs on the basis of library activities a different picture emerges:

Some two thirds of the cost are indirect costs which are used to support all the direct services in ways which are difficult to quantify. The same book can be used to support document access, information services or study facilities. This high proportion of indirect costs make cost allocation and the creation of genuine unit costs for outputs a less than exact science, where the choice of rules of thumb for allocating the indirect costs has a large effect on the apparent unit cost.

The major changes in the cost base which are foreseeable from the analysis above are three:

- less spend on buildings, but more on IT infrastructure

- a move from purchase to annual licence fees

- reduced staff costs, which means more self-service

None of these changes will make cost allocation much more reliable.

\section{The future of libraries and scholarly communication}

To look into the future of libraries, I would like to use the planning assumptions devised for a collaborative piece of work generated through the UK association of University Librarians, SCONUL. It is called "The SCONUL Vision - Academic Information Services in 2005". Six areas of planning assumptions are identified:

- Technology

- Publishing

(- Higher Education)

- Learning and Teaching

- Research

(- National Libraries)

I want to focus on the four not bracketed.

If we look at technology in 2005, the vision suggests these features:

- Increased network capacity (more images - changed publication forms?)

- Student learning and self-service administration via the Web

- University information resources available from anywhere

- Content rather than conduit

- Printing rather than photocopying

It is suggested that this technology basis will lead to the following key features of publishing in 2005:

- More scholarly publications, with open e-print archives challenging commerce

- Hybrid world of print and electronic publication and delivery

- Research "virtual communities" with access only to accepted members

- Rights management via licenses, not legislation; "fair dealing" confirmed

- Institutional debate over intellectual property rights

By 2005 learning and teaching will show these features:

- "Learning platforms" linked to other systems, internal and external

- From teaching to learning

- Combining local and commercial materials - rights management

- Work-based and private provision

- Remote learners, wider access

- Widely distributed services, centrally managed in each institution, and nationally 
- Student key skills in information handling and IT

University research in 2005 will be characterised by:

- Diversity

- Nationally-driven resource sharing

- Tension between local and other needs

- Skills of research students

\section{Why libraries have a healthy future in information management}

I believe that libraries have a very healthy future in this new world because of existing strengths and skills:

- Understanding of information structures

- Experience of large-scale service operation for diverse people

- Experience of applying technology

- Deeply embedded service orientation

- Knowledge of the community served.

All of these skills will become more rather than less useful in the future, because in the end technology alone cannot change anything, as witnessed by the many failed technological innovations. The successes in technological innovation are those which resonate with a real human need, which genuinely do something better or cheaper, or do something which could not be done before, and which find people and organisations able to apply them. Libraries as the key holders of institutional budgets for purchase of information access and with their knowledge of local needs will continue to have a pivotal role in the scholarly communication process - as have publishers, if they keep the target of genuinely better value for their customers in mind, rather than just commercial profit. 



\title{
Publication vs Communication? Searching an Identity for the University of Florence E-Press
}

\author{
Anna Maria Tammaro \\ Coordinator of Florence University Press \\ University of Florence, \\ Professor of Library and Information Science \\ University of Parma, Italy \\ E-mail address: tammaro@unifi.it, annamaria.tammaro@unipr.it
}

\begin{abstract}
Firenze University Press (FUP) started in the beginning of the year 2000 as the Digital Press of the University of Florence (Italy). It is focussed on customers, both authors and readers, attempting to eliminate obstacles and barriers to effective scholarly communication. The organisation of FUP is structured in two services: 1) pre-publication management, 2) personalisation of access. Two needs of the scholarly communication are in apparent conflict:

- the stability of the publication, including its certification and identity in the long term;

- the interactivity of communication between scholars, using the opportunities of the Web.

This involves FUP in searching an identity, transferring old communication skills to the new environment and developing new means of communication and new workflow procedures such as tele-collaboration. The benefits of the Digital Press will include greater productivity and usability with more attractive products and services.
\end{abstract}

\section{Introduction}

Firenze University Press (FUP) is a digital university press, started as a project at the beginning of 2000, inside the University Library System of the University of Florence, with the aim of supporting teachers in publishing and users in accessing the publications. The project was submitted by the University Library Co-ordinator to the Administration Council of the University of Florence and since its beginning, the Firenze University Press is found inside the Library System but with budgetary autonomy. The staff is formed of part-time librarians and technicians.

The objectives of FUP are those of:

\section{Protecting the Intellectual Property Rights (IPR) of both scholars and University}

The University should revisit the current model in which it ignores its ownership of intellectual property rights of scholarly output. The new model of an agreement between the University of Firenze and authors requires automatic granting of exclusive or non- 
exclusive licences for using the intellectual property of University teachers within the University, within a group of institutions, or within the national or international scholarly community. An agreement with the National Library of Florence has established the basis for the voluntary legal deposit of electronic publications and long term preservation of publications.

\section{Providing assistance to create, convert, and access the publications of FUP}

The choice made was that of building a digital press, with the opportunity of printing on demand the electronic publications. At the time the FUP has started, the number of publications already published by the University of Florence, using external publishers, were about 100 each year, with 30 journals on paper and with only the tables of contents (TOCs) online. In the first year FUP begun publishing two University journals, converting them to electronic journals, and about 30 other publications.

\section{Basing quality on peer review}

Peer review is greatly valued in the current system of scholarly communication as a mechanism for both quality and quantity control. However, it might be possible to see it less as an absolute prerequisite for publication, and more as a value that can be added after the publication of a scholarly work, as it progresses through the new system of electronic publication.

\section{Calculating prices on the actual direct costs to the FUP, and being predictable in costs both to authors and subscribers}

The new system of electronic publication of FUP will not be free, but we must be selffinancing. Significant costs can be removed in the electronic environment (e.g. paper, printing, packing, postage and, in the case of the University of Florence, profit). Since neither volume of material nor volume of subscribers will significantly drive costs, there should be higher predictability in costs and pricing.

\section{Assuring easiness and speediness in publication}

A scholar-controlled, networked-based scholarly communication system should significantly avoid current delays between the submission of a paper and its appearance in final form. Submissions can be "published" as soon as ready; and delivery will be almost instantaneous.

Given this background, the activities of FUP can be divided in two phases: 1) content management and 2) personalisation of access. As illustrated in Fig. 1, the activities include the full cycle of digital document, from the creation to the access.

Finally, I want to focus on two important activities of FUP that contribute to the visibility of the publications: the certification process that we guarantee for copyright and identity of copy in the long term and the metadata ${ }^{1}$ we produce for the indexing of electronic publications, in Italian and English, for better retrieval from search engines. Each publication record is sent to the national union catalogue at the National Library in

\footnotetext{
${ }^{1}$ Unimarc format is used for the record to be sent to National Library in Florence and University of Florence's central catalogue; Dublin Core for the record used for Web search.
} 


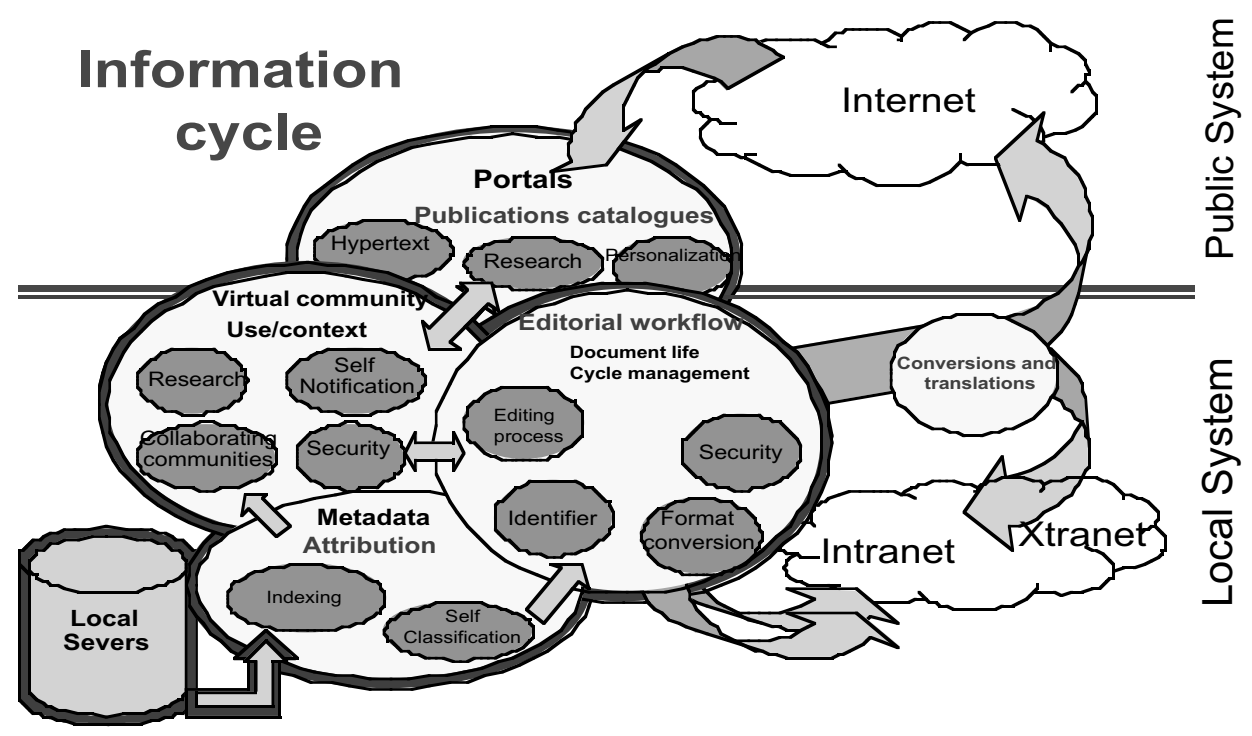

Florence and to the specialised data bases and contemporary added to the University of Florence Libraries catalogue.

The FUP is an innovative service with the aim of broadening the access to University of Florence publications. We produce services, focused on facilitating scholarly communication and not (only) publications. This means that FUP is not a traditional publisher. The application of technologies to scholarly communication is not only a change of support but as Bennett (1996) states:

“...The essential task before the academic community is not to construct an orderly economic transition between print and electronic publication. It is rather to construct an orderly intellectual and organisational transition for the certification and filtering of scholarship."

This involves transferring old communication skills to the new environment and developing new means of communication and new workflow procedures such as tele-collaboration.

What are the problems and the solutions that FUP has adopted? We list them, divided in the two working phases of FUP.

\section{Problems affecting the creation of publication}

The most prestigious scientific journals exist primarily not only to supply a short-term demand or need for information on the part of potential readers, but

"More essentially as a vehicle whereby researchers gain international esteem, recognition and, in some cases, financial reward for their work."(Hunter....)

This consideration has a big impact on FUP activities and promotion. Our first concern should be about quality of publications and we do give great attention to peer review. But we must also to consider that the most important change in scientific com- 
munication is due to the interactivity and interlinking of the Web as a channel of publication and communication. As Lyman (1997) states:

"Digital services must adapt to the communitarian nature of the network, building relationships with users"

\section{Peer review}

All papers published at FUP are rigorously peer-reviewed by experts inside the University of Florence, thus assuring the quality of publication. But some topics benefit from more wide-ranging comment before publication. To ensure these papers receive the extra review they deserve, we will post them on an e-print server to external reviewers.

Peer review was identified as the most important factor to assuage the reluctance of scholars to publish electronically with FUP. Peer review is a process that has evolved over many generations of scholars; it has become the cornerstone of academic publication and is something that is highly valued in all scholarly activities. It is important to recognise that peer review is necessarily, and appropriately, a conservative process, and that any new scholarly endeavour will take time to gain general acceptance (Cotoneschi 2001). Anyway, peer review has a strong positive effect on quality.

Quality of publications in Italy is sometimes confused with the statistical measure of the Impact factor. We cannot compete with the most prestigious publishers at the international level (and their de facto monopoly of the Impact factor where 83\% of Impact factor journals are owned by $20 \%$ of the publishers); but we believe that quality can be measured only by the experts (Tammaro 2001).

Peer review also has a beneficial impact on the quantity of scientific publications. It should be said that attempts to address the problems associated with the scholarly information explosion tend to focus more on alternative and cheaper formats for publication (Bot et al. 1998; Fishwick et al. 1998; Harnad 1996; Harnad and Hemus 1997; O’Donnel 1996) than on reducing the quantity of published research output.

The example we would like to follow is that of The Lancet $^{2}$. At the time of submission to The Lancet, authors can request that their paper appear as an eprint. Papers that pass the initial in-house editorial screen are reproduced, as submitted, on an open-access website with a citable reference indicating that the submission is unreviewed. Users of the service can read and comment on all submissions under consideration. These comments will be reproduced with the paper. Papers that appear as eprints are also formally peerreviewed. The free and formal comments are used to help The Lancet's editors decide how to proceed with a paper. There are two possible endpoints for an eprint: publication (after revision if necessary) in print and electronic formats (the citation becomes that of the printed version); or rejection, in which case the eprint is removed from the site (a record of its passage will remain) and authors will be free to submit elsewhere.

We have converted the University of Florence paper journals into electronic editions. New journals are always a risk for an author (and a reader): they have no track record, the rigor of their peer review, hence the level of quality that they certify, has not been established; their future is uncertain. At the same time, we made available at Sesto Fiorentino (the Faculty of Science campus) a depository server, particularly with the wish to make a "one-stop" shop available for deposition of some preliminary working papers by Faculty of Science authors on which they wanted comments. The inclu-

\footnotetext{
${ }^{2}$ Information about the Lancet at the journal Web site: www.thelancet.com/journal
} 
sion in this informal depository of articles submitted for formal publication in FUP journals or other publishers journals, would provide an informal complement to the formal refereeing/editorial process. Each draft paper would normally be placed in the depository at the time of submission, unless the author and the publisher agreed that this might adversely affect both parties by reducing future sales of a refereed and edited version. On approval for publication it would be removed from the depository and transferred to the journals of publication. There would be no charge for access to the depository, though Harnard suggests that some societies might wish to restrict this to members (Harnad 1995).

\section{Electronic resources encourage collaboration}

The depository of e-prints, together with the ease of communication by e-mail and a myriad of other on-line opportunities, make collaboration easier than it has ever been before. Collaboration may come in indirect ways: a researcher reads an article on-line and decides to send a response to the author because it is more immediate than writing a letter and less intrusive than a telephone call; this is clearly creating new types of scientific discourse (Abate 1997).

The FUP as "publisher", would like to organise a full electronic process of refereeing, selection and editing and would publish the articles which have survived this process in the electronic journals. The editor of an electronic journal could add peer comments alongside the final published version. However, going-it-alone on such ventures will require substantial investment in technology and skilled personnel to create value-added products. The choice of FUP was that of selecting a supplier of Web-based publishing and services. The partnership with Casalini, offering these skills and technology, allowed FUP journals to be immediately on line.

\section{Intellectual Property Rights (IPR) and integrity of digital publications}

Authors generally want the assurance that their work will not be altered by others after its publication (Tagler 1998). Boyce et al. (1997) state that :

"Readers must have confidence that the copy of the article they read has not been tampered with and that it represents what the author wrote and what the editorial process has vetted."

While alteration of the original is remotely possible with printed works, the problem is magnified for digital documents (Lynch 1994; Johnson 1997). An article printed in a traditional scholarly journal has a certain fixity. It cannot be altered without a great deal of trouble, and such alteration would most likely be readily apparent. However, a digital document is not necessarily so stable. The solution of FUP was to sign an agreement with the National Library of Florence for a voluntary legal deposit of all electronic publications of FUP. The National Library then gives the FUP a certification of the deposit and assures the long term preservation of publications.

FUP is continuing to work in the area of copyright reform, particularly as it applies to the reproduction and networking of electronic information. We are reviewing the intellectual property policies of the University of Florence trying to ensure that they best promote the transition to the emerging electronic paradigm. Such policies should address how the academic community can retain the highest possible degree of control over its intellectual property while at the same time maintaining its ability to publish where it sees fit. A set 
of different contract models have been prepared including different possibilities for authors to give or limit licence to FUP to use their works.

\section{Formats for archiving electronic publications}

A wide range of formats are available for publishing and archiving electronic journals (Cochenour \& Moothart 1995; Duranceau et al. 1996; Boyce et al. 1997). Some authors express preferences for one format over others, but there seems to be no clear consensus as to which is best. The simple ASCII text was prevalent in early electronic journals, but it suffered because it was unable to express complex mathematical formulae or symbols.

FUP uses the PDF (Portable Data Format) format because it appears to be the current de facto standard in electronic publishing. This format reproduces an actual printed page in electronic format. Therefore whatever can be printed should be able to be stored or viewed in PDF format. This format does not, however, take full advantage of the possibilities electronic publications offer, including links to references, navigation within articles, and the smooth flow of information on a screen. Boyce et al. (1997) found that users of their electronic astronomy journal chose to access articles in HTML versions five times more frequently than PDF versions. These authors believe PDF and the similar PostScript format function more as the equivalent of an electronic document delivery system. They further state that an electronic journal without links to references should not even be taken seriously.

FUP is trying to study new advanced, truly electronic versions of publications. But this effort will require time for developing the right authoring tool for authors and also for educating authors in the different ways of writing on the Web. The development of this advanced opportunity will be done by small steps. The University of Florence has plenty of motivation for taking these small and relatively inexpensive steps as, for example, installing open archives and providing their authors with the technical proxies to selfarchive their papers for themselves where needed.

\section{Access phase}

The second phase of access is focused on customers (meaning academics as authors and readers), trying to personalise the access and the management of contents, experimenting new products in the packaging of information and tailoring the contents for the different needs of users. A promotion activity was the first effort carried out by FUP.

\section{Awareness activities}

The new service of FUP requires an activity of socio-economic support to a better understanding of all the factors involved in scholarly communication. For example a comprehension of the reasons why Universities' annual serials costs are weighing down the library budgets and why the most prestigious journals in many fields are also the most expensive, although is an honour for professors to be asked to sit on their editorial boards. In general it is necessary that all teachers have an enhanced awareness of the problems created by the existing scholarly communication system, which is deeply entrenched, and galvanise the academic community around certain actions to address these problems. From a survey we have done within the University of Florence, it was evidenced that teachers need to know how peer review hiring, tenure and promotion practices are necessary to improve the impact of their scientific works on scholarly communi- 
cation and that it is necessary to experiment new form of interactivity and communication between scholars and students.

\section{Add on features}

Digitisation has created a situation where the delivery methods for electronic information have become progressively independent of the final rendering. Information can be packaged by the publisher in a variety of forms, delivered through alternative channels, for viewing on whatever device the user has available.

New access management possibilities are opened and FUP staff, as librarians expert in access, has the ambition of improving the distribution of publications. Access, delivery and personalisation require new methods and tools and in particular stress the need of cooperation. FUP is experimenting new access tools inside the Project DAFNE, financed from the Minister of Research for a national infrastructure for Italian universities publications.

Personalising content is seen by FUP with the emphasis shifting from content management (creation of publications) to personalisation of access and, at a service level, to address web-based services. This will include: automated content packaging, web-based systems, agent services, new business models, standards for interoperability and privacy. The benefits will include improved asset productivity, user control and personalised access to products and services. The aim is to facilitate the formal communication through certified publications and the informal communication, through a distributed network of e-print servers.

Availability of abstracts and indexing in English, peer comments and differential charging for browsing, all should reduce the risk that publications fail to attract potential customers. Publications of FUP could be accessed in one of three ways: (i) by subscription, either to the entirety of the stock or to selected items within it (particular subjects, "titles" etc) (ii) by direct payment, by account-holders or credit cards or (iii) a combination of the first two. Special rates for members might be applied. Competition between different publishers of this kind would keep charges down. The market is also contestable: there are few barriers to entry by new providers of services of this kind. What we are trying to reach is a better utilisation of new technologies by all, with better access to publications with attractive and effective services. The print on demand is an option between many others from which the user can choose.

The access, transmission and personalisation of large collections of digital documents require an alternative model and personalisation system to be experimented. We are experimenting new forms of the management of digital contents as, for example a database of imagines of architecture and bibliographic databases.

\section{Wider and more rapid access}

These add-on features may eliminate the cost-savings from straight reproduction of the same material in electronic rather than paper form, but the savings to the user in both cost and time may be substantial. One of the big advantages of the electronic medium is the chance of more rapid access.

Even if a journal is stocked locally in paper form, the only available copy may be in use. In the absence of access to a paper copy, use of a networked remote source is quicker and more cost-effective than alternatives such as document delivery or travel to a library owing the particular title. 


\section{Conclusions}

The opportunities of electronic publications are considered very important by the University of Florence which is investing a lot of effort in the innovative services of FUP.

In the medium term, it is necessary for University of Florence to:

Promote partnerships which apply new networking technologies to scholarly communication. These partnerships should include the university libraries, the academic staff, the computer centres, and the other university presses, as well as scholarly and learned societies, the publishing community, the government and organisations in other countries;

Make academic communication statistics more comprehensive, consistent and comparable so that a better picture of trends and problems can emerge. Complex as this undertaking will be, it is important that these statistics measure access capabilities and not simply resources and inputs.;

Encourage, fund and support current and future initiatives and proposals, such as those exploring the development of truly electronic journals.

The long-term goal of FUP is the establishment of a national decentralised, networkbased publishing system, controlled by Italian universities themselves, that retains the best practices (e.g. peer review) and eliminates the worst (e.g. excessive pricing) of the old scholarly communication system.

\section{References}

Abate T. 1997 Publishing scientific journals on-line. Bioscience 47: 175-179.

Bennett 1996 Just-in-Time Scholarly Monographs. JEP 4 (1) [Available online http://www.press.umich.edu/jep/04-01/bennett.html].

Bot et al. 1998 The cost of publishing an electronic journal. D-Lib Magazine November.

Boyce P.B., Owens E., \& Biemesderfer C. 1997 Electronic publishing: experience is telling us something. Serials Review 23 (3): 1-9.

Cochenour D. \& Moothart T. 1995 Relying on the kindness of strangers: archiving electronic journals on gopher. Serials Review 21(1): 67-76.

Duranceau E., Lippert M., Manoff M. \& Snowden C. 1996 Electronic journals in the MIT libraries: report of the 1995 e-journal subgroup. Serials Review 22 (1): 47-61.

Fishwick F. 1995 The costs facing publishers in Baum. L. (Ed.), Book publishing in Britain, London: Whitaker.

Harnad S. 1990 Scholarly Skywriting and the Prepublication Continuum of Scientific Inquiry. Psychological Science 1, 342-343 (reprinted in Current Contents 45: 9-13, November 11, 1991). [Available online

URL: ftp://princeton.edu/pub/harnad/Harnad/harnad90.skywriting].

Harnad S. 1991 Post-Gutenberg Galaxy: The Fourth Revolution in the Means of Production of Knowledge. Public-Access Computer Systems Review 2 (1): 39-53.

[Available URL: ftp://princeton.edu/pub/harnad/Harnad/harnad91.postgutenberg].

Harnad S. 1996 Implementing Peer Review on the Net: Scientific Quality Control in Scholarly Electronic Journals. In: Peek, R. \& Newby, G. (Eds.) Electronic Publishing Confronts Academia: The Agenda for the Year 2000. Cambridge MA: MIT Press. [Available online URL: ftp://princeton.edu/pub/harnad/Harnad/harnad96.peer.review.html].

Harnad S. \& Hemus M. 1998 All Or None: There Are No Stable Hybrid or Half-Way Solutions for Launching the Learned Periodical Literature into the Post-Gutenberg 
Galaxy. In: Butterworth, I. (Ed.) "The Impact of Electronic Publishing on the Academic Community" London: Portland Press [Available online http://cogsci.soton.ac.uk/harnad/Papers/Harnad/harnad97.hybrid.pub].

Johnson P. 1997 Libraries and the preservation of electronic information. Technicalities 17 (6): 8-10.

Lyman P. 1997 Digital Documents and the Future of the Academic Community [Available online http://arl.cni.org/scomm/scat/lyman.html].

Lynch C.A. 1994 Rethinking the integrity of the scholarly record in the networked information age. Educom Review 29 (2): 38-40.

O’Donnell J. 1997 Thinking Strategically about Electronic Publishing, Conference on scholarly communication for the next century, held in Vancouver, 5-8 March [Available online http://ccat.sas.upenn.edu/jod/jod.html].

Tagler J. 1998 The electronic archive: the publisher's view. Serials Librarian 34 (1/2): 225232. 



\title{
"Reti Medievali": An Initiative of New Communication of Historical Knowledge
}

\author{
Andrea Zorzi \\ Professor of Medieval History \\ University of Florence, Italy \\ E-mail address: zorzi@unifi.it
}

\begin{abstract}
Reti medievali was started in 1999 as a joint research project of five Italian universities, completely home-made by researchers. The Reti Medievali (RM) project offers text, working tools and analyses of historiography and aims to be highly informative. RM authors and users needs for information and correlated editorial organisation are described.
\end{abstract}

\section{Introduction: Reti Medievali (RM)}

I am going to speak about an on line publishing initiative I started in 1999 with some colleagues of other Italian universities (professors Pietro Corrao, Roberto Delle Donne, Stefano Gasparri and Gian Maria Varanini). I think that this experiment can be characterized as home-made: with all the advantages and all the disadvantages that this dimension involves. Just for this reason, my participation in this round table will perhaps contribute to clarify the point of view of scholars who intend to employ new technologies of communication in order to bypass the organizational and economical barriers that condition the traditional press channels of communication of historical knowledge, and in order to favor a wider distribution of personal publications. The quest for innovative alternatives disclosed by the digital press, raises in fact new problems for scholars who decide to become personally engaged in the preparation and in the editing of this new means of publication.

\section{RM contents}

First of all, I will illustrate -in a general way- our publication and the results we have obtained till now, in order to offer an idea of the contents and the shapes in which it is articulated. Its title is Reti Medievali. Iniziative online per gli studi medievistici (Medieval Networks: online initiatives for medieval studies) ${ }^{1}$. It is a cooperative initiative between

\footnotetext{
${ }^{1} \mathrm{URL}:$ <www.retimedievali.it>
} 
five major Italian universities (Florence, Naples, Palermo, Trento and Venice), initiated in 1999, which brings together online resources for medieval studies with a wide context, emphasizing the qualitative selection of material. In fact Reti Medievali [RM] is a set of web pages that analyses and promotes the use of computer techniques in the study of medieval history. The editors are teachers and scholars from the five participating universities.

The RM project plans to be highly informative by offering texts, working tools and analyses of historiography, along the lines of present-day European and Italian research and teaching practices. We also operate a selection of materials and texts submitted to the peer-reviewing. This project aims to create an on-line community of medieval scholars of unrestricted specializations, and to encourage institutions and individual scholars to experiment with the potential offered by new communication technologies ${ }^{2}$.

RM is gradually developing integrated projects: it is an electronic journal, a resource archive, a digital library, an information bulletin, a place for multi-media and cyber-space teaching experiments and a historical archive. The project is divided into six main sections. RM Journal contains debates, hyper-text essays and multi-media experiments, reviews, working-paper proposals, up-to-date bibliographies and web-site information ${ }^{3}$. RM Repertory is a structured and critical overview of the basic resources for a broad spectrum of medieval studies; it will present new material as it becomes available on-line ${ }^{4}$. RM Library publishes on-line texts concerning the fields and problems of current medieval research, becoming the first specialized library of this kind ${ }^{5}$. RM Calendar supplies information on international meetings, seminars, congresses and historical or archaeological exhibitions in the field of medieval studies ${ }^{6}$. RM Didactics presents teaching materials and experiences connected to the use of on-line multi-media technologies ${ }^{7}$. RM Memory is a sort of dictionary of scholars, of past and present works, as well as of important current problems in historiography ${ }^{8}$.

A section called RM News highlights the new input of the different sections, so as to simplify consulting the site ${ }^{9}$. We also send a monthly newsletter of updating materials via e-mail to a large Italian and international address book ${ }^{10}$. We also offer an English version of home and introductory pages of every sections ${ }^{11}$. RM is in fact multilingual: texts are written in the authors' original languages (obviously in Italian, but also in French, German, Spanish and English). Every section and every page is totally free access and full-text.

\section{RM authors/users}

RM was started by a group of scholars who felt uneasy with the current fragmentation of historiography and research. Though it could be looked at as a source of growth, this

\footnotetext{
${ }^{2}$ Cf. also <http://www.dssg.unifi.it/_RM/RM-Home.htm>

${ }^{3}$ URL: <http://www.dssg.unifi.it/_RM/rivista>

${ }^{4}$ URL: <http://www.rm.unina.it/repertorio>

${ }^{5}$ URL: <http://www.lett.unitn.it/_RM/biblioteca>

${ }^{6}$ URL: <http://www.dssg.unifi.it/_RM/calendario>

${ }^{7}$ URL: <http://www.dssg.unive.it/_RM/didattica>

${ }^{8}$ URL: <http://www.rm.unina.it/memoria>

${ }^{9}$ URL: <http://www.rm.unina.it/novita>

${ }^{10}$ The newsletter is also published on URL: <http://www.rm.unina.it/novita/Nov-archivio.htm>

${ }^{11}$ URL: <http://www.storia.unifi.it/_RM/RM-Homenglish.htm>
} 
fragmentation may cause an increasing lack of communication among the various medievalist sectors, as well as between these and other fields of historical research. The promoters recognize the need for a critical re-evaluation of traditional research and the objectives of current activities. RM hopes to intervene in the areas where the most interesting developments have occurred in Italian and international medieval studies of the last decades.

RM's challenge is to experiment fully with the potential of all new telematic and cybernetic communication systems, mostly unexplored as a community initiative. The promoters want to change the general opinion about the low scientific value of current electronic publications, encouraging an active involvement in the now undeniably global transformation of research practices and language. We believe it to be worthwhile, therefore, to pursue this service, to offer a meeting place open to all and a site for the development of new methods of communication among Italian and international medievalists.

In effect, we are receiving growing scientific acknowledgments from many interlocutors in Italy and several European countries. The statistics of contacts to RM are encouraging. In the first year of publication (may 2000-april 2001) we have registered approximately 100,000 contacts, with a monthly average at the eve of 2001 of approximately 12,500 opened pages, which indicates a trend of approximately 150,000 contacts for the current year $^{12}$. Readers are obviously mainly Italian, but with meaningful quotas of readers from the German linguistic area, from France, Spain and Latin America. Maybe, the multilingual prospect is rather penalizing if one considers that the majority of English speaking readers are mainly monolingual: in fact, we register rather few readers from England and from the USA. The analysis of data, moreover reveals an interest that characterizes RM as a working set of web sites (contacts happen mostly in the working days, with a drop in the summer and in vacation time), with a public articulated in a nucleus of specialists (teachers, researchers, PhDs and students) and in a galaxy of readers not specialist, but amateurs of the Middle Ages (a category of readers that we consider important for our initiative).

\section{$\mathbf{R M}$ architecture and organisation}

Our initiative is also physically dislocated on several servers: currently on those of the history departments and the faculties of letters at the universities of Florence, Naples, Trento and Venice, and, in a near future will be dislocated on that of Palermo, too. We aim at a better distribution of the editorial job and at giving a reticular dimension to the initiative. For the input of data and documents, each server is assisted by young editors who add to their proficiency in medieval history the competence in computer science required to carry out the job of digital editing ${ }^{13}$.

It is evident - first of all to us who are the editors of RM - that our publishing initiative, the way it is organized at present, has homemade characteristics. As I said, this has doubtless advantages: control of every phase of the work, lack of any publishing mediation, control of the costs, prompt updating, and so on. Nevertheless, initiatives like oursborn spontaneously in groups of researchers and in scholarly communities - will have to face many important problems in the near future. Among which I indicate four, at least.

First of all is the necessity to choose: whether to maintain direct control of every publishing phase (a possibility that a medium such as Internet offers like no other up to now

\footnotetext{
${ }^{12} \mathrm{Cf}$. statistic reports in every section's home page (click on icon at bottom)

${ }^{13} \mathrm{Cf}$. the board of editor's page: < http://www.rm.unina.it/redazione/Red-Redattori.htm>
} 
ever could), or to delegate the technical aspects to a specialized publishing staff?. When we put RM on line, Firenze University Press did not yet exist. It is evident that we shall have to find some sort of collaboration with the latter, because its staff will be able to supply a very important technical and editorial support. But, I ask myself if, as an example, the eventual adoption of the publishing FUP logo won't in a certain measure condition the reticular pluralism of our initiative, seeing that it was conceived as a collaboration between more athenaeums and also that it is strategically dislocated on more servers? This is a question I am asking.

Another relevant issue is the preservation of our publication. I do not refer as much to the technical aspects of safeguard and duplication of information, which we think we shall be able to face by means of techniques of mirroring, as to the legal aspects of certification and guarantee of authenticity. The recent agreement between the University of Florence and the National Library of Florence for the voluntary deposit of digital scientific publications represents an important occasion in order to guarantee these scopes ${ }^{14}$. But in this case, too, I ask myself: how could aspects like the updating, review and speed of information be guaranteed? This is also another question I am asking.

A third issue, that derives in part from the previous ones, concerns the metadata of identification of the content and the bibliographical classification of the electronic publications. I'm not an expert on the subject, but, as an editor of an on line publication, I perceive the emergency to deal with the problem. Anyhow, in this case, too, I raise a question: must initiatives like ours that were born spontaneously outside of the traditional editorial practices by force be condemned to lack a bibliographical identity unless they accept to be filtered through a publishing house? It would seem to me a serious case of marginalization, not to say of discrimination.

Finally, the issue of protection of the rights of intellectual property. I confess that it is a kind of problem I do not perceive to be quite as dramatic as it appear to all the publishers, for obvious economic reasons. From the start the RM policy has been to guarantee free access and with no charge full-text reading, without limitations of any economic sort. This policy is surely suggested by the home-made nature of direct editors of the scientific community that we represent and of our initiative. I ask myself if it would have been the same if we had passed through the mediation of a publishing house. This is another open question concerning the relationship between scholarly communication and academic presses.

\footnotetext{
${ }^{14}$ URL: <http://www.unifi.it/e-press/accordo.htm>
} 


\title{
A Framework for Italian Digital Libraries: The DAFNE Project
}

\author{
Jacopo Di Cocco \\ Professor and Director of CIB \\ University of Bologna, Italy \\ E-mail address: dicocco@cib.unibo.it
}

\author{
Laura Tallandini \\ Professor of Biology \\ DAFNE Project \\ $\mathrm{CAB}$ \\ Univerity of Padua, Italy \\ E-mail address: laura@civ.unipd.it
}

\author{
Anna Maria Tammaro \\ Coordinator of Firenze University Press \\ University of Florence, \\ Professor of Library and Information Science \\ University of Parma, Italy \\ E-mail address: tammaro@unifi.it; annamaria.tammaro@unipr.it
}

\begin{abstract}
The DAFNE (District Architecture for Networked Editions) Project, is derived from the synthesis of three separate projects elaborated respectively by the Universities of Padua and of Florence, that of the University of Bologna and that of the National Library of Florence, aimed at realizing some of the meaningful segments of the product/process defined as the Digital Library. This project has the electronic resource and its distribution/visibility within national and international services as its primary objective. The publication of the Bando Parnaso by the Ministry of Education, University and Research (M.I.U.R.) offered the occasion for proposing an integrated project by these three universities and the National Library in alliance with the firms that assumed the industrial risk of the complete project. The DAFNE Project now has the following components involved in it: the 5 initial proponents of the project: University of Padua, University of Bologna, University of Florence, Istituto di Documentazione Giuridica/CNR in Florence, National Library of Florence; 5 firms-proponents: Parco Scientifico Tecnologico Galileo in Padua, Ariadne s.r.l. in Pavia, Fratelli Bassilichi SpA in Florence, Casalini Libri SpA in Florence, Editrice Bibliografica SpA in Milano; other companies: Silver Platter Italia SpA; Consorzio Interuniversitario di Calcolo; Cineca in Bologna; La Biblioteca del Mulino SpA in Bologna.
\end{abstract}

\section{Introduction}

The technological revolution, which is in progress in the sector of informatics and telecommunications, has created the conditions of a new environment for the realisation of the digital library, as a collection of digital resources organised for a service focussed 
on users. Italian actors on information management (librarians, teachers, publishers, suppliers and information providers) - urged by the increasing market demand - are seeking a resolution to the problems which have caused a delay in the development of a strategy for the digital library in Italy.

New technology is a good stimulus for change, together with the new competition arena built by Internet. Different actors are changing traditional roles: libraries are not the only point of access to information, publishers offer direct access to end users, university presses try to develop new distribution channels of publications. The user can choose the information service best for his needs. The European Union regulations, in particular, are stimulating the change of the legislative rules for intellectual property rights management.

\section{Italian context}

In the last few years, many organisations in Italy have begun to explore the concept of a digital library. These include the National Library of Florence, the Ministry of Cultural Heritage, the Ministry of Education, University and Research (M.I.U.R.), some local organisations, the Discoteca di Stato and the Italian Libraries Association (AIB). The first efforts have been devoted to build a digital collection of rare books, electronic journals, images, and sounds. The digitising activities have not been coordinated at national level until now, but many librarians hope to plan a systematic process of conversion of the many old collections in the Italian libraries.

Project Manuzio was the first digitalisation project, based on the voluntary effort of the Association Liber Liber ${ }^{1}$, which has realized the digital version of classics of Italian literature.

The National Library of Florence ${ }^{2}$ has digitised all the works and manuscripts of Galileo Galilei and is now adding the Table of Contents (TOC) of current books to the National Bibliography.

The CIBIT, Biblioteca Italiana Telematica ${ }^{3}$, is the biggest project, co-ordinated by a group of Italian literature teachers and financed by the M.I.U.R. Its aim is to collect the most important Italian texts of every subject and type from every time. In some cases, the digital edition is the only available version of these texts.

The Discoteca di Stato ${ }^{4}$ is participating to the European Project Bibliotheca Universalis and is responsible of digitising Italian music.

Italian partners are also involved in European Union financed projects and others international projects. For example, two digital collections have been realised for mathematics community $\left(\mathrm{EULER}^{5}\right)$ and for the computing community $\left(\mathrm{ECDL}^{6}\right)$.

\footnotetext{
${ }^{1}$ The homepage of Liber Liber is available online at the URL: http://www.liberliber.it

${ }^{2}$ The digital collection of National Library is available online at the URL: http://galileo.imss.firenze.it/ multi/luoghi/firenze/bibliote/inazion.htm (visited 28/04/00)

${ }^{3}$ CIBIT is available online at the URL: http://cibit.humnet.unipi.it/ (visited 28/04/00)

An article was written by Amedeo Quondam, and Mirko Tavoni, «Storia della lingua e informatica», in Storia della lingua italiana e storia letteraria, Proceedings of the I conference ASLI, Florence, 29-30 May, 1997, Nicoletta Maraschio and Teresa Poggi Salani eds, Florence, Cesati, 1998, pp. 141-148

${ }^{4}$ Information is available online at the URL: www.librari.beniculturali.it/struttura/discoteca.htm (visited 28/04/00)

${ }^{5}$ Information about EULER is available at the URL: www.unifi.it/Biblioteche/ (visited 28/04/00)

${ }^{6}$ M. B. Baldacci, S. Biagioni, C. Carlesi, D. Castelli, C. Peters, Una Interfaccia Europea di una Biblioteca Digitale Internazionale is available online at the URL: http://giove.cnuce.cnr.it/hcitaly/ BIAGIONI-S.html (visited 28/04/00)
} 
Publishers are also investigating the digital library concepts. In particular the most advanced experiences of electronic publishing are those of Casalini ${ }^{7}$, Giunti ${ }^{8}$, Il Mulino ${ }^{9}$, Laterza $^{10}$.

All these digital collections can be used separately, searching the different systems. There is no integration with the Italian national catalogue, called SBN - Servizio Bibliotecario Nazionale (National Library Service), or with the national and international bibliographies.

In this phase, Italian libraries are starting to organise a digital collection, acquiring digital resources from vendors. Subscriptions are decreasing, especially subscriptions to scientific journals. The new electronic journals have posed new strains on libraries' budget. Consortia are developing acquisition plans in co-operation: the Consorzio Interuniversitaro Lombardo per l'Elaborazione Automatica (CILEA) in Northern Italy is the most influential consortium while the Consorzio Interuniversitario per le Applicazioni di Supercalcolo per l'Università e Ricerca (CASPUR) in the South is the second for importance. Both, CILEA and CASPUR are consortia of universities, adding new information services to the others offered to academic partners. The Italian National Forum on Electronic Resources (INFER) is an Italian alliance of librarians for information and support in negotiation with publishers.

New alliances were made with suppliers, as intermediates for negotiation or as gateways to different publishers' digital collections. The library services of the Universities of Bologna, Florence, Genoa, Padua, Venice and the European University Institute in Florence have been committed for a long time to independent projects, but with similar background and goals, to develop and integrate their libraries' services. They have a strong interest in technological developments that could enable integration of database network systems with direct access to periodical literature available in electronic format. Within this framework, the above mentioned libraries' services have discussed the possibilities of further cooperation and have reached a formal agreement to set up a pilot project, named CIPE - Cooperazione Interuniversitaria sui Periodici Elettronici (Inter-university Co-operation for Electronic Journals) with the purpose of gaining access to scientific information in electronic format thus ensuring a high level of consistency with the more advanced international standards and the best possible results in terms of economic costs and usage rights and provisions.

This new commitment is effectively projected in the potential digital exploitation (organisation and management of delivery) of the accessible information using interactive models - as far as possible not pre-structured - guaranteeing where possible the diffusion/distribution of the information itself in real time with respect to the request.

All digital resources acquired or created by digitising are accessed through the Web. This naturally implicates a reconsideration of the telecommunications web, so that this can be seen on the one hand as an essential infrastructure which makes the diffusion of on-line services requesting the "goods-information" effectively possible, and on the other as a medium in the true sense of the word, that is a means which enables acceleration of information distribution, promoting new forms of communication/commerce and conditioning the processes which generate them. This becomes more significant in proportion to the extent to which the information sources of a system, organisation or organism,

\footnotetext{
${ }^{7}$ Available online at the URL: www.casalini.it (visited 28/04/00)

${ }^{8}$ Available online at the URL: www.giuntimultimedia.com (visited 28/04/00)

${ }^{9}$ Available online at the URL: www.mulino.it (visited 28/04/00)

${ }^{10}$ Available online at the URL: www.laterza.it/internet/ (visited 28/04/00)
} 
however this may be structured, are distributed in a capillary manner over an extended and heterogeneous territory.

This reconsideration of the concept of the web poses a problem, which is complex to resolve. It involves administrative, cultural, political, economic and social aspects, and demands considerable efforts on the part of public bodies and institutions as well as by private and social operators so that the emerging need can be satisfied. It is in fact very clear that only a strong and conscious affirmation of the web, both as an infrastructure and as a medium, will guarantee the capacity of our country's system to respond to the challenges of the new global market. Electronic documents constitute a growing part of the cultural heritage, and offer users notable benefits. The growth of digital information is changing the way in which information is created, managed, transmitted and exploited.

More specifically, the vision of the world of digital information is centred on the user. The removal of intermediaries will considerably reduce the costs of the information distribution chain, with significant consequences in terms of an awareness of users' needs, the identification and localisation of information, and the economic and juridical aspects of book commerce. For this purpose we have to accelerate the completion of the infrastructure, stimulate the production of electronic documents and the realisation of research systems which can identify, make accessible and distribute the electronic resources. This infrastructure is the aim of the DAFNE project.

\section{The DAFNE project (District Architecture for Networked Editions)}

The importance of the DAFNE ${ }^{11}$ project lies in the realisation and fine-tuning of a complete structure which allows the user to obtain access to information and document retrieval, prefiguring the National Digital Library and enables the producers of goods and services - even if these are frequently entities of limited size - to take advantage of the "Italian district of electronic publishing".

The objectives of the DAFNE project are:

- to realise an Agent for licensing

- to plan the digitalisation of collections at national level

- to build an Index to Italian scientific periodicals

- To co-ordinate the development of the University presses and the collaboration with publishers.

The DAFNE project has 5 academic proponents: University of Padua, University of Bologna, University of Florence, Istituto di Documentazione Giuridica/CNR (Institute for legal documentation/CNR) at Florence, National Library of Florence. It also has 5 private firms as partners: Parco Scientifico Tecnologico Galileo (The Galileo Science and Technology Park) at Padua, Ariadne s.r.l. at Pavia, Fratelli Bassilichi SpA at Firenze,

11 J. Di Cocco, L. Tallandini, M. Vedaldi, I: Il Progetto Parnaso "DAFNE": Cogliere le opportunità offerte dai nuovi standard generali per l'informazione in rete. Presentazione del Progetto Dafne al Salone Internazionale dell'Arte e del restauro e della Conservazione dei Beni Culturali e Ambientali- Ferrara, 2629 March, 1999

L.Tallandini- M. Vedaldi: Il Progetto Dafne: Salone del Libro giuridico. Naples, Istituto S.Orsola Benincasa, 6 June, 1999

L. Tallandini: Il Progetto Dafne: Collegamenti e integrazioni con la realtà SBN, ICCU- SBN Notizie 1999 , in press 
Casalini Libri SpA at Firenze, Editrice Bibliografica SpA at Milano. Other partners are: Silver Platter Italia SpA, Consorzio Interuniversitario di Calcolo (Cineca) at Bologna, La Biblioteca del Mulino SpA at Bologna. The DAFNE project will come to an end in 2003.

The central role of the user who has access to universal electronic resources is nevertheless in contrast with the physical, administrative and political development of the isolated local structures. It is therefore necessary to establish an equilibrium between the local priorities, the interests of publishers and the common objectives of national interest.

A complete integrated circuit, as well as constituting a model of administrative infrastructure which includes all the interested parties (from the author to the user) also promotes the production of electronic documents through small- and medium-sized Italian scientific publishers, which on their own would not be able to face the competitive demands emerging from a market in continual evolution. In fact, the specificity of electronic publishing on the web in respect to that in other mediums (including CD-ROMs) as well as the priority distribution channel for electronic publishing created by electronic commerce, necessarily implies a continual updating of the instruments and standards adopted and investments which the circuit redistributes and shares among all the users at a pre-established price. We have only to consider the difficulties of integration and insertion in other integrated circuits of proprietary products and services.

\section{The structure of the product/process}

The DAFNE project aims at providing the technical-administrative infrastructure and the instruments necessary for allowing the individuals and entities involved to carry out, in an efficient and effective manner, within the context of a global market all the operations envisaged by the supply-demand circuit for electronic documents in Italy. It also promotes the integration of the two Italian districts of Italian publishing accessible on the Web and that of the digital library. For this reason, as well as integrating existent instruments and services, it is also aimed at developing those not yet available.

The graphic below (fig.1) represents the overall structure of the district, the blocks which compose it, and the principal flows between the entities involved in the whole circuit of the production and circulation of electronic documents .

We can observe a horizontal axis, which, from west to east, displays the flow of publications and electronic documents from the producers towards the distributors (commercial and/or library) and from these to the users. DAFNE will concern itself with organising this flow, which will take place via the Web, and will arrange all the technical, economic and administrative solutions necessary in order that the users may have easy access to the publications, or to a single part of them (for example a single article from a journal).

The second axis is that of the services; this goes from north to south and is centred on the distribution system. The intangibility of the support and the transmission medium makes it indispensable to augment the role of the information systems (inclusive of libraries) and at the same time increases the importance of electronic commerce in line with the trend to immediately access only the texts which have effectively been requested at the moment of the demand.

This group of three blocks has to inter-collaborate through the exchange of a series of messages between open systems. This requires an in-depth analysis of the functions, the operators who perform them and the real (even if intangible) and monetary flows between them. An independent non-profit organisation would be a single focal point for negotiations and central brokerage, eliminating unnecessary costs and duplication, leveraging resources and promoting standards. Thus the different initiatives of digitalisation will hopefully take the form of institutions actively working to make resources which they own and to which 


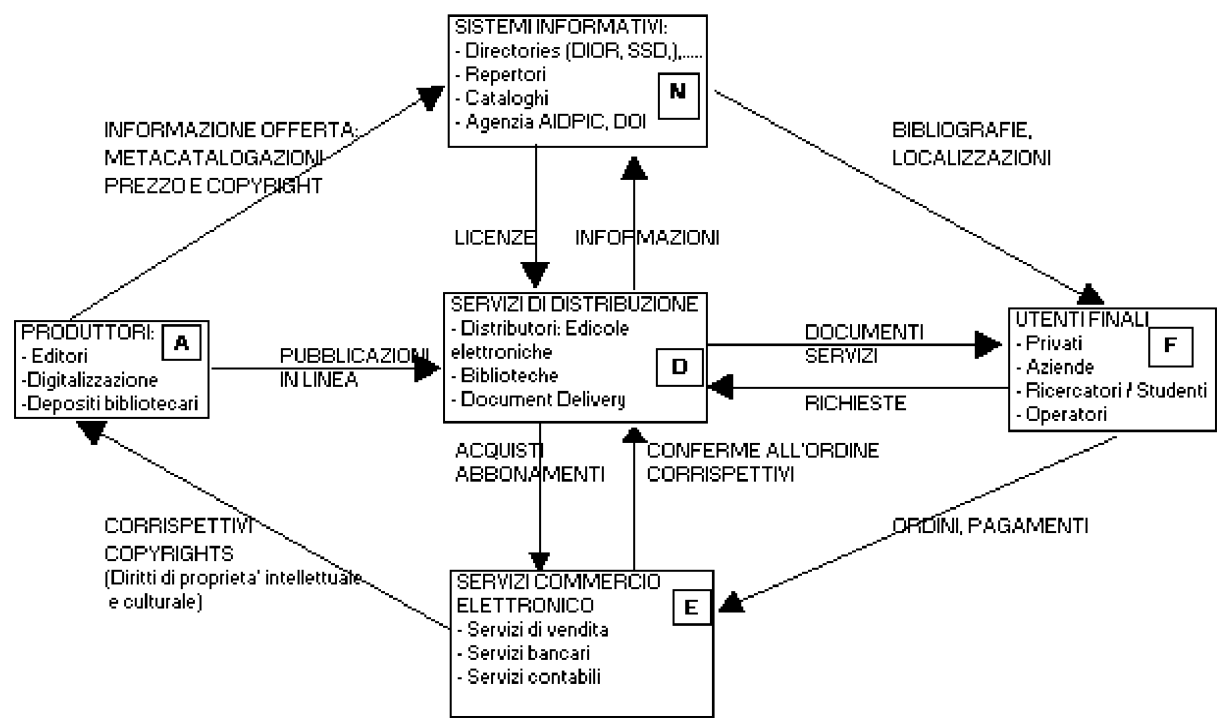

Fig. 1. Demand-supply circuit of on-line publications

they have legal copyright, available in electronic format and gives this digital library the rights to make these resources available to its patrons in a non- exclusive manner.

The upper arc between producers, information services/systems and users shows the flow of information necessary for a correct knowledge of the offer on the part of the users, a market transparency and a coherent and pertinent choice. In the world of intangible goods, such as electronic documents, correct, complete and easily accessible information assumes an essential role in valorising and guaranteeing competition for editorial products, and in providing a satisfactory response to the information request of the user.

\section{DAFNE Interoperability}

The DAFNE project defines interoperability as the ability of digital library components or services to be functionally and logically interchangeable by virtue of their having been implemented in accordance with a set of well-defined, publicly known interfaces. In this model, different services and components can communicate with each other through open interfaces, and clients can interact with them in an equivalent manner. When repositories and digital objects are created in this manner, the overall effect can be a federation of repositories that aggregate content with very different attributes, but which can be treated in the same manner due to their shared interface definitions.

Our approach to interoperability has three fundamental principles behind: (1) agreement on common abstractions, (2) definition of open interfaces to services and components that implement the common abstractions, and (3) creation of an extensibility mechanism for introducing new functionality into the architecture without interfering with core interoperability. The basic services will be:

Search services: Searching across domains presents particular challenges given their different underlying content models and descriptive standards. Different search services may be provided: for textual material, for image content, and so on. 
Request/order services. These manage the request transaction from placing a request to its successful completion. They need to interwork at technical and business levels, and communicates with accounting, billing, authentication and other services. There is some standardisation in the university area, with the CINECA electronic portfolio.

User interface services. Services may be presented in different environments: webbased, immersed, or through some form of visualisation. Services may share some basic services and infrastructure within agreed frameworks for communication.

For example, resource discovery services may report on the availability of services, may use location services to identify instances of resources (mirror sites for example), may be combined with user profile or rating services to refine selections, and so on. A service which mediates access to the holdings of several memory organisations might provide support for discovery and selection of services, manage service requests, translate formats, aggregate services, consolidate results, manage authentication and financial transactions, and so on.

Some development areas will be:

Resource discovery services. These will support the discovery and selection of services and resources. An example of a resource discovery service is a "subject gateway" for social sciences.

Terminology and knowledge representation services. These may provide vocabulary support for query expansion or indexing. Participating institutions have invested significantly in knowledge representation activities to support discovery or metadata creation.

Authentication services. In the new shared-space of the Internet, users and services may have no prior knowledge of each other. Users, services and resources may need to be authenticated to provide assurance that they are what they purport to be.

E-commerce services. Partners of the DAFNE project will increasingly provide chargedfor services, and common approaches will be needed.

Caching and mirroring services. These services will become more common later in the Project, assisting the economic use of network resources.

Some services of the DAFNE project will begin to be shared in the social sciences area, using some existing finding aids. For example:

Legal and economic databases. These are existing citation descriptions, and will provide infrastructure for support of distributed access, use and preservation of resources.

Location services. We use this phrase for the resolution of identifiers in locations. Persistent identifiers will be valuable in a range of application contexts. The scenario of DAFNE project is one, which is being explored in a library context and in a market context, where multiple copies of items may exist. The development and deployment of identifiers, and their interworking, present significant R\&D challenges.

User profile services. These may be necessary for personalisation services, and store data about user permissions, profiles, and behaviour. Third party services may emerge, and there are clear links to authentication and other services. How to characterise user preferences, behaviour and privileges in acceptable and useful ways is an open question.

\section{Conclusion}

The emergence of so many interested groups in digital library exploitation suggests that enough of the enabling technologies are now available in Italy to try new forms of information creation, management and distribution. By keeping abreast of new developments in the digital library, different actors are trying a new strategic alliance. We are not far enough along in the transition to a fully electronic environment to expect that this model will act as an umbrella for the entire set of information needs of our society. 



\title{
The Limits of Innovation: A Publisher's View About The Impact of Digital Publishing on Academic Communication and Research Diffusion
}

\author{
Enrico Francesconi \\ Researcher \\ Department of Informatics \\ University of Florence, Italy \\ E-mail address: enrico@mcculloch.ing.unifi.it
}

\begin{abstract}
In spite of the original enthusiasm, digital publishing growth is facing difficulties and uncertainties. Especially in the academic area, the opinion about the low scientific value of existing electronic publications has greatly limited the development of digital publishing. Moreover, the technical problems, which still persist in digital library creation from paper publication archives, discourage the investments in electronic publication consulting frameworks. This fact causes a loss of interest, also in the academic area, for electronic publications, which basically are inaccessible from university library systems. In this paper the status of digital publishing is discussed from an academic publisher's point of view, as related to the technical obstacles towards electronic documents availability, which is strictly linked to the reliability of document digitalising systems.
\end{abstract}

\section{Introduction}

Academic publishing places particular confidence and hopes in electronic publishing.

However, the transition from a traditional academic publishing, based essentially on paper versions of the publications, to a new academic publishing mainly based on electronic publications, faces structural limits and organizing difficulties. Basically these problems are related to the uncertain economic profits from electronic publications and to the technical adequacy and support for the production and fruition of new digital publishing productions or of electronic publications that derive from paper archives.

In this paper these kinds of limitations to the diffusion of electronic publishing in academic communication are discussed.

This paper is organized as follows.

In Section 2 the economic aspects related to electronic publishing in the academic area are discussed. In Section 3 the technological limitations and the level of maturity of document digitalising systems are illustrated. Finally, in Section 4 some conclusions and perspectives, related to the possible development of electronic publishing in the academic field, are reported. 


\section{Economic aspects in digital academic publishing}

After an early period of wide enthusiasm about the potentialities of digital publishing, in particular regarding the possibilities of free access to publishing for researchers, the times of disillusion arrived. In fact it became clear that digital publishing doesn't mean no cost publishing: a lot of fixed costs (such as editing, proof reading, paging, royalties, promotion) still remain in the needs of a publisher and for a digital publisher too.

It means that the publisher needs funds for publishing and these funds have to arrive from sales and/or subventions. In this way the original framework of the academic publishing process recurs, in the sense that publication and diffusion are limited by the costs level. Consequently, the main problem for a digital academic publisher basically is the same as that of a traditional academic publisher: promoting and selling the books till the publication costs and acceptable profits are gained.

Moreover, a digital publisher also needs a certain amount of printed books. This is because he has to fulfill legal obligations in terms of deposited copies. Moreover there are cases in which some paper copies are needed for a university competition or book reviews and promotion.

Finally authors themselves require having their electronic books also in paper version. This is because books uniquely in electronic version are considered of lesser scientific value in comparison with traditional books. This is due to the inflation of low level drafts that characterized the first phase of electronic publishing.

Indeed, in electronic publishing a cost reduction does exist. It is represented by the disappearance of printing costs, but, in the chain production, it is not so relevant as other and more significant costs which could be reduced in electronic publishing: they are represented by distribution costs.

For an academic publisher university libraries and researchers are the main potential customers. Therefore, the existence of a computer network inside a university library allowing the use of electronic books could permit the maximizing of the convenience of using an electronic format.

Unfortunately, the technical problems, which still persist in digital library creation from paper publication archives, discourage the investments in electronic publication consulting frameworks. This fact causes a loss of interest, also in the academic area, for the electronic publications, which basically are unaccessible from the university library systems.

\section{The level of maturity of document processing systems}

As discussed above, the implementation and growth of digital libraries for the academic environment represents the main strategy for developing academic electronic publishing. One way to face the problem of digital library creation from paper archives, in fact, is to acquire the information by converting each document into an electronic format, but the information that they contain is difficultly retrieved in a selective fashion.

Therefore, digital library construction deals particularly with the creation of databases of information extracted from documents or systems capable of storing and retrieving information from databases of intrinsically multi-media documents, where paper is just one of the media.

Hence the need exists to have document reading systems able to classify and understand documents aimed at extracting data from documents and creating a database; a 
database represents a more nimble consulting tool and ensures a better data rationalization and compression with respect to document storage in the electronic format.

The development of automatic document reading systems, therefore, is a topical problem for digital library implementation as well as in all those fields where data contained on paper need compression, translation or transformation to different media. In the last few years a growing interest has arisen in the artificial intelligence community to build up systems which are able to process documents, since document digitalising is different from being simply a problem of OCR reliability: it is a typical artificial intelligence problem that involves image analysis, namely low-level image processing techniques, and, from a higher level of abstraction, problems of machine learning, knowledge representation, semantic labelling of objects extracted from a document image, and statistical reasoning.

A document, in fact, can be described with respect to its physical structure [1], [2], [3], called also layout structure, and its logical structure [3].

According to [3] the layout structure of a document is the collection of the extracted objects, obtained by the repeated division of the content of the document into increasingly smaller parts, on the basis of the presentation. An object of the layout structure is called physical object [3]; it is essentially a rectangle including a part of the document image.

Similarly, the logical structure of a document is the collection of the extracted objects, obtained by the repeated division of the content of the document into increasingly smaller parts, on the basis of the human-perceptible meaning of the content [3]. An object of the logical structure is called logical object [3].

A document processing system is aimed at extracting the layout structure of a document (document analysis) and at mapping it into its logical one (document understanding) [2].

The design of systems for particular reading tasks on single classes of documents with a fixed layout has reached a consolidated level of maturity [4], [1], [5] (Fig. 1). Such systems present a considerable reliability but they usually lack flexibility, that is they are concerned with the task of multi-classes of document image understanding.

A more limited number of works deals with the problem of system flexibility. The systems aimed at flexibility usually use a knowledge-based approach to document process-

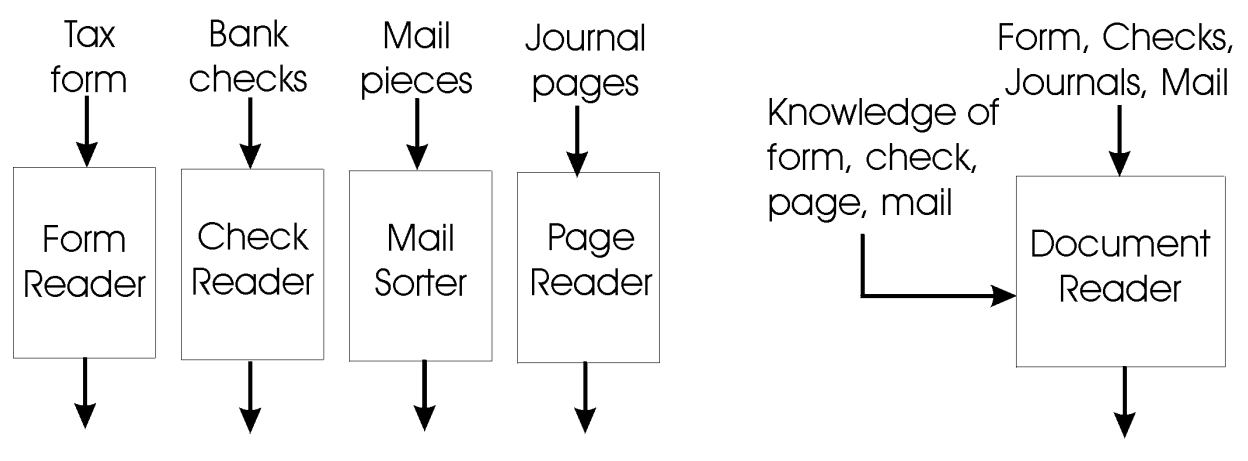

Fig. 1. Closed and Open Document Processing Architectures [6] 


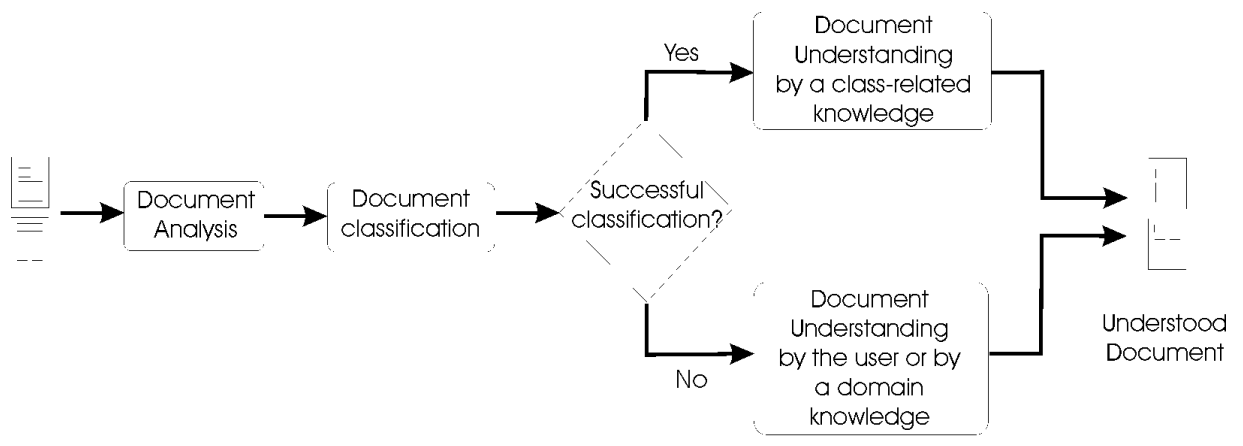

Classification-based Architectures

Fig. 2. Classification-Based Document Processing Architecture

ing. The knowledge on a domain is used directly in the understanding phase without a predefined goal of reading, in order to set up open systems [6], [7], that can be adapted to read different types of documents (Fig.1).

Other systems are intended to realize a compromise between reliability and flexibility. They aim at an enlargement of the domain of documents to be processed, preventing a great decrease of reliability. They are intended to individuate a number of classes in a document domain of interest, so as to limit the layout variability a system has to face (Fig. 2).

Moreover, they introduce the possibility of recognizing the class a document belongs to and of performing the document understanding by tools well-suited to documents of the recognized class, [8], [9], [10], [11], [3].

The analysis of the literature and our experiments showed that, at the state of the art, only the systems aimed at processing documents of a particular domain exhibit good results.

Our experience has been addressed to developing neural networks-based OCR systems [12] and to the problem of multi-class document processing [13], [14], [15]. In particular we have proposed a knowledge-based architecture for processing documents of a multiclass domain using a classification-based architecture, and a method for the construction of the knowledge, used to understand documents, by means of a small learning set of the domain documents.

Our experiments showed an average percentage of successful multi-class document object recognition of $83 \%$ [16].

\section{Conclusions}

There are not so great differences between the works of the traditional publisher and the digital publisher in the academic field. However, the economic conveniences of an academic publisher can be improved by producing electronic publications. Digital publishing in fact allows for the reduction of printing, stocking and good movements costs. 
Particularly, there is a desire to reduce distribution costs: in academic publishing the solution is represented by the implementation of digital libraries.

At present, technical problems still persist in digital library creation from paper publication archives. Both the literature and our experience show that only the system for processing documents of a particular domain exhibit good results. This fact discourages the investments in electronic publication consulting frameworks, since they cannot be provided by a meaningful number of electronic publications, therefore conditioning electronic academic publishing.

For these reasons, at this moment it is impossible for an academic publisher to survive as a pure digital publisher. The only solution is to act as a traditional publisher, ready to transmigrate to digital production as soon as it becomes convenient. This strategy is feasible today thanks to print-on-demand solutions.

\section{Bibliography}

[1] Tang Y.Y., Suen C.Y., Yan C.D. \& Cheriet M. 1995 Financial document processing based on staff line and description language. IEEE Transactions on Systems, Man and Cybernetics, 25 (5), 738-753.

[2] Tang Y., Yan C.D. \&. Suen C. 1994 Document processing for automatic knowledge acquisition. IEEE Transactions on Knowledge and Data Engineering. 6 (1), 3-20.

[3] Esposito F., Malerba D. \& Semeraro G. Multistrategy learning for document recognition applied artificial intelligence. An international journal, 8 (1), 33-83.

[4] Cesarini F., Gori M., Marinai S. \& Soda G. INFORMys: A flexible invoice-like form reader system. IEEE Transactions on Pattern Analysis and Machine Intelligence, vol. 20, 730-745, July 1998.

[5] Ha T.M. \& Bunke H. 1994 Model-based analysis and understanding of check forms. International Journal of Pattern Recognition and Artificial Intelligence, 8 (5), 10531081.

[6] Lam S.W. 1994 An adaptative approach to document classification and understanding. Document Analysis Systems, 231-252.

[7] Lipshutz M. \& Taylor S.L. 1995 Functional decomposition of business letters. Symposium on document analysis and information retrieval, 435-448.

[8] Dengel A., Bleisinger R., Hoch R., Fein F. \& Hones F. 1992 From paper to office document standard representation. IEEE Computer, 63-67.

[9] Watanabe T., Luo Q. \& Sugie N. 1995 Layout recognition of multi-kinds of table-form documents. IEEE Transactions on Pattern Analysis and Machine Intelligence, 17 (4), 432-445.

[10] Walischewski H. 1997 Automatic knowledge acquisition for spatial document interpretation. Proceedings of the International Conference on Document Analysis and Recognition, 243-247.

[11] Esposito F., Malerba D., Semeraro G. \&. Pazzani M. 1993 A machine learning approach to document understanding. Proceedings of the Second International Workshop on Multistrategy Learning, 276-292.

[12] Francesconi E., Gori M., Marinai S. \& Soda G. 2001 A serial combination of connectionist-based classifiers for ocr. International Journal on document analysis and recognition, 3 (3), 160-168.

[13] Cesarini F., Francesconi E. Gori M. \& Soda G. 2000 Using physical and logical constraints for invoice understanding. Pattern analysis and applications, special issue on document image analysis and recognition, 3 (2), 182-195. 
[14] Cesarini F., Francesconi E., Gori M., Marinai S., Sheng J.Q. \& Soda G. 1997 Rectangle labelling for an invoice understanding system. Proceedings of the International Conference on Document Analysis and Recognition, 324-330.

[15] Cesarini F., Francesconi E., Gori M. \& Soda G. 1999 A two level knowledge approach for understanding documents of a multi-class domain. Proceedings of the International Conference on Document Analysis and Recognition, 135-138.

[16] Cesarini F., Francesconi E., Gori M. \& Soda G. 2001 Analysis and understanding of multi-class invoices. International Journal on Document Analysis and Recognition. Submitted. 


\title{
JOP. Journal of the Pancreas: a model for the dissemination of scientific information and sharing of knowledge in the field of Pancreatoloy and Diabetology
}

\author{
Raffaele Pezzilli \\ Medicina d'Urgenza e Pronto Soccorso \\ Ospedale Sant'Orsola \\ Via Massarenti, 9 \\ 40138 Bologna (Italy)
}

\author{
Generoso Uomo \\ Unità Pancreas \\ Ospedale A. Cardarelli \\ Via A. Cardarelli, 9 \\ 80131 Naples (Italy)
}

\author{
Antonio Maria Morselli-Labate \\ Department of Internal Medicine and Gastroenterology \\ University of Bologna \\ Via Massarenti, 9 - 40138 Bologna (Italy)
}

\begin{abstract}
This contribution describes the creative process of a highly specialized scientific journal, published only in electronic format. This model presents totally innovative aspects regarding both its content and the policy adopted for access to it and the intellectual property of the works published in it. In particular the experiences and difficulties that were encountered in the planning stage and the initial diffusion of the periodical will be reported. We will illustrate the aspects which make a periodical that is published in only electronic format different, since it does not appear in the catalogue of any famous publisher nor is it financed by subscriber's fees, To resolve this problem the objectives of covering costs through sponsership have been set.
\end{abstract}

Scientific communication in the field of Medicine is regulated by mechanisms that, in many cases, are still dictated by the traditional publication process that we have experienced through the paper journals.In the Internet era this process often fails to serve the new emerging needs for a rapid and efficient dissemination of knowledge.

Too frequently in the recent experiences we have seen elements that cause unnecessary delays in the dissemination of information, some examples are: long publication times that in some cases can even take six to twelve months from submission of paper to its publication

Impact Factor as authors try many journals where they can publish starting from those with a high IF and this delays the publication of their paper, sometimes independently from the quality of it. In some cases they choose as a first journal to try the one with the highest IF even if it is not the one that has the major relevance with the content of their paper) cost of traditional journals, even when available in e-format, as not everyone 
can afford the high cost of subscriptions and consequently have regular access to the information there published.

JOP. Journal of the Pancreas in a new highly specific journal in the field of pancreatology and allied sciences published in electronic format only. It is an independent, not for profit journal and is freely accessible via the Internet.

We strongly believe in the importance for Authors to retain the intellectual property of the articles that they send to journals as this facilitates the scientific communication and exchange. This is why we decided that the intellectual property of the original articles published in JOP stays with the Authors and we believe this to be one of the most innovative aspects of our initiative.

When we first conceived the idea to create it we decided to keep a format that could be very similar to the paper edition of a traditional journal. In other words we decided to adopt a structure that would be divided into issues then grouped in annual volumes, where every volume represents one full year.

JOP has been assigned an as ISSN number (1590-8577). Currently we are investigating the aspects connected with the deposit of a legal copy of it in the Italian National Library.

JOP first issue was made available on-line on May $10^{\text {th }}, 2000$. Other 5 bimonthly issues have been made available since then. All of them were accessible on time as of the $10^{\text {th }}$ of the month of publication.

The table of contents of every issue of JOP is electronically distributed to more than 20,000 pancreatologists and diabetologists who subscribed to JOP Newsletter.

JOP's scope and coverage specifically concentrate on very defined areas of clinical medicine - such as the pancreatology and diabetology - with a big emphasis on research studies. The quality of content is granted by the very strict, and still extremely fast, peer reviewing process that is based on the higher level of requirements of the most renowned traditional journals. The JOP Editorial Board is composed by eminent scientists in the field from many different countries who are responsible for ensuring that one of JOP fundamental principles is fulfilled: to disseminate quality knowledge rapidly in order to contribute to the advancement of science for the benefit of the whole community.

Throughout the first year of publication we had the opportunity to collect and evaluate comments received by scientists worldwide who expressed their opinion on our initiative. In the majority of cases the feedback was highly positive and we have had confirmation that a sort of new method for the dissemination of knowledge in our field was needed.

One point of major concern is represented by the fact that JOP is not yet included in A \& I databases and specifically has not yet been assigned with an IF value. The latter seems to still be a very important element for Authors in order for them to decide to which journal to submit their paper. Although rated as a very important element IF has not to date stopped those Authors who decided to submit their contributions to JOP to publish with us.

JOP has started an evolution process and in the future it will probably change its format in order to continue to serve the emerging needs of its readers and Authors. One first example of this gradual mutation is the hosting of a virtual round table that was published in the January dedicated issue of JOP.

Another example is represented by the publication in the September issue of the Proceedings of a Conference, each paper completed by its slide show or video.

One year from the birth of JOP we can say that what we considered as a big challenge -to be faced keeping in mind Seneca's words "It isn't because things are difficult that we don't try, but by not trying, things become difficult"- represents today a clear example of the possible and necessary changes to the models adopted so far for the dissemination of scientific information and, as such, for the sharing of knowledge. 
Yet this successful initiative suffers from its most important aspects: its being a freestanding freely accessible resource. These aspects make it very difficult for us to find the necessary funds to cover the ongoing maintenance costs.

We are currently exploring ways to offer added value services -to be charged to those interested-in order to fund the overall initiative. 



\title{
E-Communication among Italian zoologists: Two Years of Experience with "Vertebrati" Mailing list
}

\author{
Claudio Gnoli \\ Secretariat Board \\ "Vertebrati" \\ E-mail address: vertamm@bigfoot.com; gnoli@aib.it
}

\begin{abstract}
Vertebrate zoology in Italy represents a good example of a community of scholars improving communication using Internet. The history of the list "Vertebrati" is illustrated and its development.
\end{abstract}

\section{Introduction}

Electronic communication is already well spread in some disciplines, like e.g. physics and biotechnology, with a wide use of tools like e-journals, preprint servers, web sites etc.; while some other disciplines, like e.g. agricultural sciences, veterinary, and field zoology, are less strictly related to information technology, and generally slower in developing a daily use of its tools. Spreading of access to the Internet among the population also varies in different countries. So, some communities of scholars sharing a common field of study may be potentially interested in getting in contact, though being not yet organized in networks. Vertebrate zoology in Italy can represent a good example of a scope where communication is capable of being improved by Internet tools.

\section{Birth of the list}

For such reasons, some members of Centro Studi Faunistica dei Vertebrati -a research section of the Italian Society for Natural Sciences, based at the Natural History Museum of Milan- having some experience with electronic mailing lists, have imagined applying the consolidated technology of list servers to create an Italian discussion group on Vertebrate zoology. Such an idea became real in early 1999, thanks to an agreement with CILEA (Consorzio Interuniversitario Lombardo per l'Elaborazione Automatica), which provided its list server, working with the PMDF Mailserver, to host the group.

The list was christened "Vertebrati", to clearly express its precise scope, its scientific approach, and its Italian base (although postings in English would have been accepted too). As the basic setting, it was decided that: 
- everyone could subscribe to the list, without need of any approval by the owners (people caring and controlling list operation);

- messages could be sent to the list only from subscribed addresses, even in order to prevent unsolicited commercial postings (spamming);

- messages could be sent directly to the list, i.e. there were no moderators;

- the default reply address was that of the list, rather than that of the sender (such choice, though typically producing some noise of wrong replies, encourages a use of the list for discussion, rather than just for distributing isolated news).

The main way to promote the list was collecting email addresses of zoologists, who were publicly available in the web sites of most zoology departments of Italian universities, and of some other zoological and environmental institutions. Addresses of mailing lists devoted to related topics were also looked for, but the main Italian resources of this kind appeared to be limited to the birdwatchers list "EBN Italia", plus a recently born network among nature parks. A single announcement was sent to such addresses, and although it was unsolicited, only one person out of several hundreds protested about it. Instead, some tens of people, included well-known researchers from several universities, followed the instructions to subscribe in the following weeks, and the list began to effectively work from April 1999.

\section{Progress of the list}

First postings were some calls for petitions and congresses, and requests for information on the Italian distribution of some Felidae species. There was of course no guarantee that the group would have been viable and the subscribers would have found it worth. However, the list passed the critical threshold of the first weeks successfully, and stabilized around an average of several messages per week. The dimension of the chosen scope (Vertebrate zoology on a national scale) turned out to be adequate to produce an amount of traffic consistent enough to keep the list viable, but at the same time not so high to become hard to follow for most subscribers. As a proof, without any more promotion on a large scale, the number of subscribers has slowly grown during the life of the list, to reach about 300 at the beginning of 2001 .

Such a delicate equilibrium is not granted to all: a great number of discussion groups evolve instead either toward an almost complete absence of participation, or toward an exceeding abundance of messages, many of which are off-topic, offensive, or unoriginal. Eugenio Gatto, owner of one of the most successful discussion groups in our country with today more than 2700 subscribers and a regular traffic ("AIB-CUR: Italian librarians" <http://www.aib.it/aib/aibcur/aibcur.htm3>), reports that the trend of its first years was similar to that of "Vertebrati". The experience of the mentioned group proved also very useful to manage several technical issues for "Vertebrati" (see below).

Among the most relevant subjects emerged during the first year, there was a prolonged thread about the management of the Grey squirrel Sciurus vulgaris, an alloctone species whose presence in North-Western Italy is threatening the survival of the related autoctone one Sciurus vulgaris: the National Institute for Wildlife (INFS) had planned an attempt of eradicating the former by trapping and killing animals, but had been sued by animalist organizations for that; the question was having a big echo in the newspapers. In such a situation, "Vertebrati" acted as an important place for getting non-sensationalist information on the subject: some researchers explained the scientific reasons for such an unpopular plan, and discussed it with other subscribers. The list owners agreed with researchers from Turin University to publish some documents concerning the project on 
a web server and the following action <http://www.cilea.it/vertebrati/doc/sciurus.htm>, and a motion in support of the involved scientists, subscriptions to which were collected by the list owners and sent to INFS. Some other petitions for important questions of environment management were also spread through the list.

Other topics often discussed concerned news on the distribution of some species, especially large mammals like the Wolf; poaching; common errors and distortions by mass media in giving information about wildlife; management of alloctone species with special reference to freshwater fishes; requests and answers about the use of software for statistics, geographical information systems, and other technical instruments like radiocollars and traps; announcements of congresses and other events.

\section{Technical issues and management}

As a typical feature of mailing lists, some technical problems occur even in "Vertebrati", which need to be managed by the list owners, in order to keep communication as efficient as possible, and avoid a progressive loss of subscribed addresses for casual reasons.

- Some copies of each message are bounced from subscribed addresses that are temporarily or definitively not working. Error messages arrive at an address of the owners, who can then ask the postmasters of the mail servers hosting the addresses for information; unfortunately many of them don't answer. Addresses not working for many weeks are finally un-subscribed by the owners.

- Messages sent from addresses that are not subscribed to the list are sent to an address of the owners; most often, they happen to be proper messages from people not yet subscribed or subscribed from a different address: then owners resend them unchanged to the list. Unsolicited advertisements, instead, have been almost absent until now.

- Instructions to subscribe and unsubscribe are automatically sent to all new subscribers with a welcome message; however, some people ask for them anyway by writing to the owners or to the list: the right procedure then has to be taught them, or directly performed (the latter is quicker but less constructive).

- The commonly accepted rules for a fair use of mailing lists (netiquette) are also sent with the welcome message; however some people violate them, by including in their posts off-topic information, text in non standard formats, large attached files, etc., which can cause serious problems to receivers, especially to those with poor and expensive equipment to be connected to the Net. Owners try to manage such cases, by evaluating whether they are worth sanctions against the sender, like his forced unsubscription; however they try to be indulgent, as many cases depend on lack of skill or carelessness; and to limit their intervention to a minimum, not to producing the feeling of an authoritative control over the list by few people. After a virus was spread through the list in early 2001, it was decided to limit the maximum size of messages at 10 Kbyte, so as to block most attached files.

To process such issues, a small mailing list ("VertAmm") was also activated, allowing for quick communication among the owners, who are qualified as list secretariat. The Secretariat board currently includes Carlo Biancardi, Anna Rita Di Cerbo, Claudio Gnoli, Achaz von Hardenberg, Edoardo Razzetti, and Paolo Zubiani. They work mainly to decide about problems like those described above and the development of the list. Decisions must be made in a relatively short time (within a few days after the problem originated), but at the same time in a non-rash and well-balanced way: so secretariat members generally discuss before acting, but in urgent cases each of them is also allowed 
to intervene directly, while other members can be temporarily far from their email access, by writing to the list or by sending commands to the server. The list for secretariat board was realized with one of the best mailing list services freely available on the Net ("OneList", later merged with "eGroups", later merged again with "Yahoo!"), as the advertisements automatically included in messages by such services are not a problem in this case. Subscribers of "Vertebrati" are encouraged to write to the "VertAmm" address, for technical help or other questions about the list.

\section{Survey on the use and impact of the list}

In early 2001, subscriber Armando Nappi suggested to the secretariat board doing a survey on subscribers' satisfaction and opinions about the group. The secretariat adopted the idea and discussed details of possible questions. Eventually, a questionnaire was released and sent to all addresses subscribed to the list, asking them to send answers to an address of the owners. 112 people replied before the deadline, and their answers and comments were analysed and published <http://www.cilea.it/vertebrati/inchiesta.htm>.

The sample of subscribers resulted to be mostly:

- aged between 30 and 49 years $(66 \%)$

- possessing a degree (59\%), most often in Biological Science or Natural Sciences

- interested in zoological research from a professional point of view (68\%)

- interested mainly in postings on Mammals and/or Birds

- using email occasionally to discuss about wildlife (47\%)

- subscribed to "Vertebrati" for more than 1 year $(67 \%)$

- posting on an average less than one message per month $(78 \%)$

- satisfied with the current management of the list (91\%)

- thinking the current number of subscribers to be satisfactory for Italy (59\%)

Professional composition of the subscribers sample resulted to be quite various: 17 university students (mostly in Natural Sciences), 6 graduated, 6 holders of grants, 10 researchers or employed in the university, 9 researchers in agencies other than the university, 6 working in parks, 5 working in naturalistic or scientific museums, 3 wildlife managers, 2 collaborators of universities, 3 school teachers, 13 privately employed in the environmental or zoological field, 17 others.

Most people (47\%) think that $75-100 \%$ of discussed topics fit the list, and only $(63 \%)$ would prefer not to receive $0-25 \%$ of them; "messages scarcely relevant for the main aims of the list, like asks for addresses or literature references", should be sent anyway according to $53 \%$, and "only if one really was not successful with other ways" according to another $44 \%$. So subscribers generally seem to be quite tolerant about the content of messages.

However, out of 24 people having sent suggestions for the management of the list, 16 were concerned with ways to control it more; among them, 4 would like the default reply address to be only that of the sender, and 2 would like the list to be moderated. Other wishes were concerning a wider range of discussed topics, a systematic discussion on topics suggested by the owners, and the use of standardized prefixes in the subject field.

Most people $(63 \%)$ think that such a kind of lists are very useful to support the exchange of opinions about Italian wildlife. Some (18\%) have even developed useful cooperations with other people contacted through the list. A specific question about whether mailing lists can "make scientific communication simpler by avoiding the long path of publication in specialized journals" had well distributed answers: many people think that they cannot, "because there is no filter on the quality of information (peer 
review)" (51), and "because researchers are not willing to share knowledge that they would rather publish" (35), while others think they can (29). But many highlighted that we should not look at these two tools as competitors, since mailing lists are more devoted to exchanging opinions and practical information, even if of uncontrolled origin; while traditional paper publications are slower but public, and they involve accuracy, quality control, and persistence of information; "the equivalent of a peer-reviewed journal can be realized by web rather than by mailing list: <http://www.oikos.ekol.lu.se/we/we.index> for an example", an expert user points out. Another side of the question is the risk that researchers get tired of reading amateur questions; although until now some expert researchers are actually participating actively in the group.

\section{Future developments}

In the survey two people suggested that most interesting information posted to the list could be collected and kept in a web site. Selective archiving would be indeed one of the most interesting developments of "Vertebrati".

Some programs for list servers or web forums allow automatic creation of archives, which can then be viewed by date, sender or subject. However, many messages contain information not worth being preserved for a long time, or off-topic, or sent by fault. Another problem is that great amounts of old postings can prove useful only if arranged by subject in some way; but experience has shown that a large majority of people are inaccurate in using standard ways of summarizing the contents of their posts in the subject field: even in discussion groups for librarians, who by their profession should be especially aware of such techniques, and have been explicitly recommended to pay attention to it, subjects are found to be far from standardized... The various personal systems for email add more confusion, by inserting additional prefixes ("Re:”, "R:”, "Fwd:”, ...) in different forms.

All this suggests that, to be useful, an archive of posted messages should be selected and organized by hand in some way. "Vertebrati" owners are currently planning to create an archive of the discussions of more general and durable interest (of course avoiding any selection based on opinions), and arranging them by some system of classification. This clearly involves a considerable amount of human work, and hence can be realized only gradually in time.

When made available on the Web, postings would become public at any effect; while discussion mailing lists are not completely public, as postings are sent only to a community of subscribers, even if it can be an open one and the sender can be unaware of the identity of most receivers. So assent by the original senders should be obtained before publishing their postings.

The variety of job situations of the participants can be a value, making the group an interprofessional channel alternative to the traditional and hierarchical communities of university and other institutions. However, it seems desirable that the list keep its character more oriented toward scientific research than toward amateurs (there are other popular channels, like newsgroups, which are more devoted to hobby communication). The number of students and people not related with zoological research among subscribers could grow quickly, since email is becoming a very spread tool in these past few years, and is no more a privilege of university people. For these reasons, it seems especially important in the future to promote the list among those researchers who are still unaware of its existence, for example by leaflets distributed at congresses. 
The survival of "Vertebrati" through the critical phases of its infancy, its consolidation and its two year long experience, which even in the light of the survey results can be viewed as positive, mean that it now represents a precious resource, worth of investing renewed efforts, and of producing valuable information for the scientific community. 


\title{
Scholarly Communication and Academic Presses: the Nexsus Network Journal
}

\author{
Kim Williams \\ Editor-in-chief \\ Nexus Network Journal \\ 50054 Fucecchio (Firenze), Italy \\ E-mail address: K.williams@leonet.it
}

\begin{abstract}
The Nexsus Network Journal (NNJ) affeared in Internet in 1999 and grew out of a biennial conference series dedicated to architecture and mathematics. The idea of a journal was put forth because the research community was small and distributed worldwide. The paper describes some of the experiences and lessons learned.
\end{abstract}

The Nexus Network Journal (NNJ) for architecture and mathematics appeared on the Internet with its first issue in January 1999. It grew out of a biennial conference series dedicated to architecture and mathematics that I had begun in 1996. The conference series began as a way to bring together people working on interdisciplinary studies in architecture and mathematics. The papers presented at the conference were published in book form. As early as the second conference held in June 1998 it became clear that much more material was arriving than could be properly dealt with within the limitations of the a single biennial print publication. The idea of a journal was put forth. Because at that time our research community was quite small and because its members came from all corners of the globe, we thought that an electronic journal would best suit our needs. The $N N J$ has now outgrown the initially free space offered to it by the server and has its own domain. Having begun with a mailing list of 75 people, it has grown to 400 . The homepage of the site receives about 1,300 visitors monthly (visitors to individual pages are not counted; there are at this moment 306 pages on the site). What is particularly exciting about the $N N J$ is that we are not only establishing a new journal, exploiting the possibilities of a new medium and diffusing scholarly material of high quality, but we are defining a new discipline in the process. Before the beginning of the Nexus conferences in 1996 (organization actually began in 1995), there was no field of architecture and mathematics as such, whereas now, only 6 short years later, we not only have a field, we have a research community as well. Of course for now the $N N J$ is the only journal in its field. How would I feel if another, competing journal were established? I would feel GREAT because that would really mean that the field of architecture and mathematics had come of age.

In this paper I would like to describe some of the experiences and lessons learned throught the $N N J$, in light of some of the topics that were brought up during the day-long international conference on scholarly communication and academic presses held on 22 March 2001 in Florence, sponsored by the Firenze University Press. 


\section{Are e-journals stigmatized?}

One of the points brought up at the digital publishing conference was that the advent of a new medium had given rise to a plethora of new journals. Many of these, however, failed to take into account whether the particular field could support another journal, and many journal failures were blamed on a failure of the new digital medium rather than the failure of the journal itself. The question of status was brought up: are electronic journals as well regarded as traditional print journals? Conference participants seemed to feel that there was no stigma placed on electronic journals as such, but rather that the acceptance or lack of acceptance of e journals could be attributed to the fact that most ejournals are new journals and it takes time for any new journal to become accepted. One participant raised the question of why an author would want to submit a paper for publication to a new, unestablished journal, electronic or not, when he could submit it for publication to an already established journal of proven reputation. I was the first to answer that question, because the NNJ provides the perfect answer. It lies in the author finding, not the largest readership, but rather the right readership. Being an interdisciplinary journal, our readers belong to fields of mathematics and architecture. Given a paper that deals with, for example, the geometric analysis of an architectural monument, if it is published in either an architecture journal or a mathematics journal, regardless of how established or prestigious, the paper is only going to reach half its intended readership. This was the case with the first papers detailing fractals, which were published in a journal for meteologists and so were unknown to mathematicians for some time.

But what determines whether a new journal becomes an established journal or a failure? This is determined by how many papers are submitted for publication. If enough material arrives, then with time the journal will become established. If not enough papers are submitted for publication, then the journal will have to close its doors. In effect, as Steven Harnad expressed it, "authors vote with their papers". I am very happy to say that authors are voting for the Nexus Network Journal, because the amount of material submitted for publication continues to grow.

\section{The limitations of digital publishing}

Although the introduction of new possibilities for content is an exciting development for publishers, the Hypertext Markup Language (HTML), the present language of digital publishing, presents some serious limitations. This is particularly true in mathematics, where even the latest versions of some widely used browsers are unable to correctly read mathematical equations, rendering the paper thus published unusable. This kind of limitation means that e-journals will flourish in some fields more than others.

One way to handle the problem of mathematical equations is to convert a document in Word that includes formulas into HTML code, during which process the formulas will be automatically converted into .gif image files. One drawback of the importation of mathematical symbols and expression as graphics is that the gif files can lead to a very large document, requiring long loading times. Under development in MathML, a markup language for mathematics, but not yet widely used or available.

A workable alternative to html is the Adobe Acrobat program that produces papers in Portable Document Format (PDF), permitting both internal and external links (coordinated through a browser). The drawback of PDF files is that the reader must download the file before viewing it, and use a second program (a browser) to follow external links. Thus this alternative results in a loss of much of the immediacy that characterizes the Internet. 


\section{Open Access}

Steven Harnad demanded that scholarly papers, for which the authors receive no royalty payments, should be made available to the scholarly community 24 hours a day, 7 days a week, for free, forever. The Nexus Network Journal currently meets all of these requirements but one, which it will soon meet. Available on the Internet, access to the NNJ is unlimited, that is, is open to all with no password required, 24 hours a day, 7 days a week.

The $N N J$ is also free, with no subscription fee and no licensing fee for libraries. (However, the print version is for sale at a low $\$ 20.00$ per volume.) The $N N J$ is one of the exceptions to the rule in the field of mathematics e-journals. According to Allyn Jackson of Notices of the AMS (October 2000: 1053-1059), "there are around 675 mathematics journals in all and only about 35 of these are free electronic journals". In our case, the journal was set up in such a way to assure a proper diffusion for papers published. Charging a user or licensing fee was seen as counterproductive, given the initial small readership for the journal. Perhaps the only criticism we have had of the open access policy comes from the distributor of the print version, who maintains that the free full-text version online hurts the sales of the print version. I tend to disagree (though admittedly sales of the print version have been less than hoped for), because I believe that if a reader gains a positive experience of the journal on the Internet he will subsequently purchase the print version himself or request it of his library.

However, the last criteria demanded by Harnad, "forever", is a different story.

\section{Permanence}

First of all, because the Internet is in its infancy, as is the digital publishing medium itself, and because technology is developing at such a breathtaking speed, terms like "forever" and "permanent" take on a certain relevance. In the Nexus community we were are quite concerned about making sure that papers published in the $N N J$ be made permanently available for the sake of referencing and retrieval. Initially we explored the idea of producing an annual CD version of the journal, but this was discarded because the CD itself may be so soon superseded (who knows that today's CD's might not go the way of yesterday's 5-1/4" floppies). It was therefore decided to produce an annual print version of the journal in traditional form. Paper is still perceived as the only permanent medium, and it was thought that sales of the paper edition would make the NNJ self-sustaining (a result that has not yet been realized). The production of a print volume also solved another one of our problems, limited space on the server. Thus after about two years online and once published in the print version, papers were taken offline to make space for new material.

This caused an outcry among readers, as well as among those who linked their sites to articles in the NNJ. This problem was solved through the kind intervention of Berndt Wegner, Editor-in-Chief of Zentralblatt für Mathematik, who introduced me to Anna Maria Tammaro of FUP. The Firenze University Press will be permanently housing the $N N J$ archives on the FUP server, making them available "forever" and eliminating the need to remove them from open access.

\section{References}

First of all, as was pointed out by Anthony Watkinson, publishing consultant and visiting Professor in Information Science at City University, London, e journals are a very new 
medium. He claims to have been the first to establish an e-journal in 1993, a mere eight years ago. This means that many of the issues that arise because of the newness of the medium have yet to be definitively resolved.

These issues include knotty, everyday problems such as the proper form of citation of a paper published on the Internet. I have attempted to solve this problem by inserting a "Correct Citation" note at the end of each paper published in the NNJ, giving, in addition to the correct information for citing the article (author's name, title, volume and issue number, URL), a more or less accepted formal standard for the citation, so that those who want to cite an article published in the $N N J$ need only cut and paste the citation from the $N N J$ page to their own document. This is intended not only to facilitate references to articles published in the $N N J$, but to encourage them.

Other issues involving the referencing of web pages are less easily resolved. One involves page content. Digital publications are not only a translation of a traditional text into a new medium, but contain content that was not possible with print publications. Image content is one aspect of this; animated gif images, for instance, cannot be reproduced in print, but form a normal part of digital publications. Hyperlinks and frames represent another normal situation that complicate referencing. Interactive programs such as Modular Games by Slavik Jablan, produced for the the $N N J$, are yet another aspect of the possibilities inherent in digital publishing that are at once exciting and perplexing. Because it is possible for the reader/user of an electronic journal to determine the content of a given page through a few clicks of the mouse, the question arises of just how valid it is to cite a particular page or URL. Sites that feature virtual realities present yet another complication. As Donald Sander, president of Learning Sites, Inc (http://www.learningsites.com) wrote to me, his site features “...complete navigable 3D virtual spaces and in each space the user can call up linked datasets which are created to suit that particular search -different for each visitor, there is no URL for the retreived information, nor any way (today) to bookmark the viewer's location in the world." It is clear that this takes referencing into a new realm.

\section{Conclusion}

Although the publishing of e-journals represents "the flea on the dog" in Steven Harnad's terminology, that is, the dog being all academic publishing and the flea of ejournal representing only a small sector of academic publishing, and although the electronic revolution that was expected within a few years to bring the traditional scholarly press to its knees has not come to pass, the e-journal has assumed a role as a flexible, independent and accessible instrument for the diffusion of scholarly works. 


\title{
Academic Presses and Integrated Systems: Towards a New Perspective
}

\author{
Antonella Cosetti \\ Centro Tecnologie Didattiche Universitarie Multimediale a Distanza (CTU) \\ University of Milan, Italy \\ E-mail address: cosetti@unimi.it; office@ctu.unimi.it \\ Gian Franco Greppi \\ Professor Veterinary Medicine \\ BIOVET \\ University of Milan, Italy \\ E-mail address: Gianfranco.Greppi@unimi.it; greps@mailserver.unimi.it
}

\author{
Massimo Valdina \\ Editor \\ MG Electronic Media \& Books \\ Bergamo, Italy \\ E-mail address: maxvald@tiscalinet.it
}

Since the dawn of civilization, man has used different methods of communication from cave paintings to manuscripts to printed books.

Printing revolutionised the dissemination of information by enabling multiple copies to be made from a publication.

Now in the twenty-first century it is possible to produce a virtually unlimited number of copies of any book.

All means of expression contain qualities and characteristics, which are peculiar to it.

At every stage of the evolution of these means of communication, man has increased his ability to extend his knowledge in time and space.

Today new technologies exist in response to new needs. The reader wants quick, cheap and up-to-date information.

Quality production, development and transfer of information nowadays call for information models which are dynamic, media-orientated and creative that have to pay particular attention, on the one hand, to the needs and experiences of whoever produces the information and, on the other, to the use the reader makes of it.

In this way it is possible to have advantages from the characteristics and quality of the means of communications, which we have at our disposal.

At this stage of change the implications of the technological revolution in the cultural world are complex and not easy to interpret. The universities ?in particular public univer- 
sities? need to acquire the new technology to cope with the challenge of didactic globalisation quickly.

This means not only acquiring information technology but also developing new concepts, which are right for this.

Who are the actors of the process of information transfer? In the universities they are the authors, the publishers and the Institutions.

Each of them has his own responsibilities if the institutions (the Universities) can guarantee scientific rigour and authority whereas the publishers guarantee quality.

A contradiction has developed inside the academic world. Even if the University can potentially produce high levels of knowledge, the spread of this knowledge remains low and so the resulting products is highly expensive. The new technology can offer various solutions which differ from traditional ones and the purposes of this communication is to illustrate those alternatives by proposing a new perspective which will not lose sight of the reader, the end point of this information.

The first response to the new demands of the reader is the use of the online network for the e-book, an exclusively electronic publication. The expression of this phenomenon is scientific electronic review publishing: online reviews are, we think, a winning strategy because the review, by its very nature, needs to be updated quickly and must be easily managed without having a limit on the number of pages which a periodical inevitably has. Moreover, an electronic review enables the reader to print only the articles which are of interest to him.

A second approach is to use digital printing which, on the one hand, remains part of the traditional world of the book while, on the other, uses new technological instruments which differ from the traditional typography and which enable the institutions, and the other actors, to obtain advantages previously unthought of.

Among this are:

- the high quality of the resulting product,

- the possibility of make a small number of copies only for a particular client or institute, - careful management of the resources (Digital printing publishers don't have the problem of storing unsold copies),

- the final product on the market is economical and it is easily updated.

This is the reply to the present day needs of the world of information.

There are some people who believe that traditional books will be entirely replaced by e-books and new technology and some people who think that this will not happen.

In reality, digital printing makes Gutenberg's book a living revolution which is destined never to die.

The third approach to the problem that we are looking at today and which appears strictly connected with universities is the offer of products which are not the book but are related to it and which are not designed to substituted it but to complement it as they have characteristics which allow the reader to reach objectives which differ from those the book itself proposes.

The use of such instruments derives from the questions coming from the students who ask for the means to become protagonists of their own learning path. This happens thanks to the opportunity to access a wide choice of information, which ranges from books to audiovisual products, from multimedia offline products to those online.

In the multimedia world we see the word enriched by images, animations, laboratory simulations and links to Internet sites specialized in various university disciplines; all factors which allow for a broadening of the mind and the widening of knowledge.

There are two other aspects which digital technology permits. These are the self-testing of one's level in any subject through random testing and the possibility to follow distance learning or any communication. 
The fact that the students enjoy this new technology and the success that teaching online has had recently has led many Italian Universities to open up to innovation.

This opening up can be seen in two areas. Experimenting on the part of individual teachers or departments moved by enthusiasm or personal interest and using specialized multimedia centres already presents in the university, or as choices made by the university, which involve the entire university structure.

What we hope for in the future is not only the use of modern technology within the traditional university structures but also a radical renewal of teaching and scientific communications towards the creation of a university press capable of responding to the demands of the academic world.

We mustn't forget that the modern publisher is not very different from the old Manuzio because the attention given to details in printing a book is fundamental and cannot be abbandoned. 



\title{
Conclusions
}

\author{
Hans Geleijnse \\ Director of Information System and Services \\ European University Institute \\ Badia Fiesolana (Firenze), Italy \\ E-mail address: hans.geleijnse@kub.nl
}

I would like to thank the organizers for this. It is clear that the Digital Press of the Firenze University Press is probably the most important initiative in this field in Italy and that Florence is again a place of inspiration and innovation.

The conference has once again confirmed that librarians and authors are unhappy with the current, traditional process of scholarly publishing. We do not yet completely agree on the new business model for the future and we do not yet precisely see how to move from where we are to where we want to be in 5 to 10 years. We have to continue discussing it, at conferences and through intensive E-mail communication, but we need, above all, new initiatives and experiments.

Anthony Watkinson and Michael Keller stressed that currently publishers and libraries add important value to the information process and that they will only remain in business if they continue to do so.

In the future, the key functions in the organization of the information process will not necessarily be executed by (all) libraries and (all) publishers. Many things are in flux, many things are changing in the communication and distribution process driven by new technologies. A positive aspect of developments during the last ten years has been that librarians have shown many more activities and initiatives than many people had expected. But this is not the time to relax.

Steven Harnad was very outspoken with his proposal to make the 2,000,000 refereed journal articles that are produced every year available on everyone's desktop. Every librarian will agree with this proposal, but many publishers will be horrified.

I believe that we are rapidly moving towards a situation in which universities worldwide make their own research output available online on the Web.

Many universities, from Japan to Finland, are currently working in this direction. Librarians should actively support this development. Naturally, this implies that they should support the Open Archives initiative aiming at interconnectivity of distributed self-archived scholarly information.

Harnad was right when he stressed that putting the material on the Web is one issue, but quality control and certification is another. It is the most critical issue.

The organization of the process of peer review is currently in the hands of publishers. If they are doing a good job and if they add value to the information process at reasonable conditions, there are good reasons to maintain this system. 
Peer review should be an independent process, researchers want to have recognition by their peers. Basically this is a proven system that has contributed significantly to the development of science and technology.

Although the organizing role of the publishers should not be underestimated, the key to the whole process is in the hands of the editors, reviewers and authors - mostly academics - of the most prestigious journals. They will determine the outcome of the debate.

It would be most advantageous if universities were to stimulate the debate on the role of academics in the publishing process and on future models of publishing. It would be a good thing if universities could make their researchers aware that they do not necessarily have to give away all aspects of their copyright, without limitations and restrictions, to commercial and society publishers.

In this area a University Press can play an important role by

- developing new ways of publishing

- supporting academics in their publishing activities

- making important research publications and other information available and accessible.

From my own experience with a University Press I know that it is also a risky business. Important conditions are that

- the University Press gets the financial, organizational and political support of its parent institution

- there is a sufficient critical mass of publications

- well-known professors take the lead, are prepared to publish with the University Press and stimulate colleagues to do the same.

It would not be a good idea for every single university to start its own press and start to reinvent the wheel. If universities are planning to start a digital university press, it would be better to take advantage of the experiences of others, to cooperate, to make use of all the opportunities of our virtual environment, to share infrastructure and publishing tools. Cooperation can be very profitable and does not imply a decreased visibility of the university as shown in the Dutch Roquade project presented by Bas Savenije.

Finally, I would like to thank the University of Florence and the Firenze University Press. I wish them much success with their new initiatives. I am looking forward to cooperating with you in the future. 


\section{Contributors - International Conference on Scholarly Communication and Academic Presses}

Bergamin Giovanni, Librarian, National Library of Florence, Italy. Telephone: 055249191, E-mail address: giovanni.bergamin@bncf.firenze.sbn.it.

Cosetti Antonella, Centro Tecnologie Didattiche Universitarie Multimediale a Distanza (CTU), University of Milan, Italy. E-mail address: cosetti@unimi.it; office@ctu.unimi.it.

Di Cocco Jacopo, Professor, Director of CIB, University of Bologna, Viale Q. Filopanti 7, 40126 Bologna, Italy. Telephone: 051-2094265, Fax: 051-2094266, E-mail address: dicocco@cib.unibo.it.

Francesconi Enrico, Researcher, Department of Informatics, University of Florence, Italy. E-mail address: enrico@mcculloch.ing.unifi.it.

Geleijnse Hans, Director of Information Systems and Services, European University Institute, Badia Fiesolana, San Domenico (Firenze), Italy.

Gnoli Claudio, Secretariat Board, Vertebrati, Telephone 382-505665, E-mail address: vertamm@bigfoot.com; gnoli@aib.it

Greppi Gian Franco, Professor Veterinary Medicine, BIOVET, University of Milan, Via Celoria 10, 20123 Milano, Italy. E-mail address: Gianfranco.Greppi@unimi.it; greps@mailserver.unimi.it

Harnad Stevan, Professor of Cognitive Science, Intelligence/Agents/Multimedia Group Department of Electronics and Computer Science, University of Southampton, Highfield, Southampton SO17 1BJ, UK. E-mail address: harnad@cogsci.soton.ac.uk, http://www.cogsci.soton.ac.uk/ harnad/

Keller Michael A., University Librarian, Director of Academic Information Resources, Publisher of HighWire Press, Publisher of Stanford University Press, Stanford University, Berkeley California 94305-6004 USA. Telephone: 650-723-5553, Fax: 640725-4902, E-mail address: makeller@sulmail.stanford.edu

Morselli-Labate Antonio Maria, Department of Internal Medicine and Gastroenterology, University of Bologna, Via Massarenti 9, 40138 Bologna, Italy. Telephone and Fax: 051549653, E-mail address: morselli@med.unibo.it.

Pezzilli Raffaele, Medicina d'Urgenza e Pronto Soccorso, Ospedale Sant'Orsola, Via Massarenti 9, 40138 Bologna, Italy. Telephone: +39-051-6364701, Fax: +39-051-549653, E-mail address: editor@joplink.net. 
Savenije Bas, University Librarian, Utrecht University, The Netherlands. Telephone: +31 30-2536502, Fax: +31-30-2539292, E-mail address: b.savenije@library.uu.nl

Tallandini Laura, Professor of Biology, DAFNE Project, CAB, University of Padua, Italy. E-mail address: laura@civ.bio.unipd.it; mail.cab@www.cab.unipd.it.

Tammaro Anna Maria, Coordinator of Firenze University Press, University of Florence, Professor of Library and Information Science, University of Parma, Italy. E-mail address: tammaro@unifi.it: annamaria.tammaro@unipr.it

Uomo Generoso, Unità Pancreas, Ospedale A. Cardarelli , Via A. Cardarelli 9, 80131 Naples, Italy.

Valdina Massimo, Editor, MG Electronic Media \& Books, Via San Tomaso 74, 24100 Bergamo, Italy. Telephone: 333-4009242, Fax: 333-04009242, E-mail address: maxvald@tiscalinet.it.

Watkinson Anthony, Publisher consultant, Visiting Professor in Information Science, City University London UK. E-mail address: Anthony.watkinson@btinnet.com.

Wegner Bernd, Editor-in-chief, Zernblatt fur Matematik, Technical University Berlin, str.d. 17. Juni 135, 10623 Berlin. Telephone: +49-30 31 423616, Fax: +49-30 31 421604, E-mail address: wegner@math.TU-Berlin.DE.

Williams Kim, Editor-in-chief, Nexus Network Journal, Via Mazzini 7, 50054 Fucecchio (Firenze), Italy. Telephone: 0571-20489, Fax: 0571-22033, E-mail address: K.williams@leonet.it.

Winkworth Ian, Director of Learning Resources, Library, University of Northumbria at Newcastle UK. E-mail address: ian.winkworth@unn.ac.uk.

Zorzi Andrea, Professor of Medieval History, University of Florence, Via San Gallo 10, 50122 Florence Italy. E-mail address: zorzi@unifi.it. 